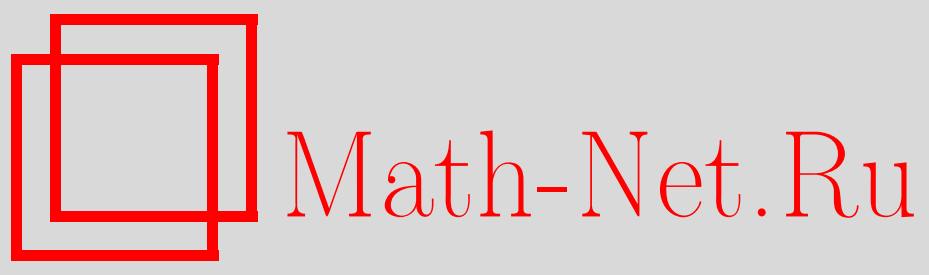

С. Д. Глызин, А. Ю. Колесов, Н. Х. Розов, Теория неклассических релаксационных колебаний в сингулярно возмущенных системах с запаздыванием, Матем. сб., 2014, том 205, номер 6, 21-86

DOI: https://doi.org/10.4213/sm8271

Использование Общероссийского математического портала Math-Net.Ru подразумевает, что вы прочитали и согласны с пользовательским соглашением http://www . mathnet.ru/rus/agreement

Параметры загрузки:

IP : 44.207 .124 .84

26 апреля 2023 г., 13:30:47

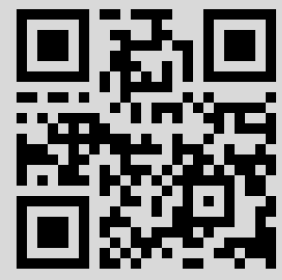




\author{
С. Д. Глызин, А. Ю. Колесов, Н. Х. Розов
}

\title{
Теория неклассических релаксационных колебаний в сингулярно возмущенных системах с запаздыванием
}

\begin{abstract}
В настоящей работе вводятся в рассмотрение некоторые специальные классы релаксационных систем с одной медленной и одной быстрой переменными. При этом эволюция во времени медленной компоненты $x(t)$ описывается обыкновенным дифференциальным уравнением, а быстрой компоненты $y(t)$ - дифференциальным уравнением вольтерровского типа с запаздыванием $y(t-h), h=$ const $>0$, и малым параметром $\varepsilon>0$ при производной по времени. Исследуются вопросы о существовании и устойчивости в указанных системах периодических решений импульсного типа, т.е. решений, у которых координата $x$ при $\varepsilon \rightarrow 0$ сходится поточечно к некоторой разрывной функции, а координата $y$ является $\delta$-образной. Полученные результаты иллюстрируются на ряде примеров из экологии и теории лазеров.

Библиография: 11 названий.
\end{abstract}

Ключевые слова: неклассические релаксационные колебания, сингулярно возмущенные системы с запаздыванием, асимптотика, устойчивость.

DOI: $10.4213 / \mathrm{sm} 8271$

\section{$\S 1$. Введение}

Имеющуюся к настоящему времени общую теорию релаксационных колебаний в многомерных системах обыкновенных дифференциальных уравнений с некоторой долей условности можно разделить на классическую и неклассическую. Классическая теория, разработанная А. А. Дородницыным, Л. С. Понтрягиным, Е.Ф. Мищенко и Н.Х. Розовым, носит достаточно законченный характер и в суммированном виде содержится в монографиях [1], [2]. Что же касается неклассической теории, то в случае релаксационных систем на плоскости она изложена в статье [3], результаты которой в расширенном варианте вошли в книгу [2]. Следует также отметить статью [4], посвященную исследованию неклассических релаксационных колебаний в математической модели реакции Белоусова.

Суть неклассической теории проиллюстрируем на простейшем содержательном примере. В качестве такового возьмем систему

$$
\dot{x}=\alpha-x(y+1), \quad \varepsilon \dot{y}=\left[x-1-\frac{\beta}{1+\gamma y}\right] y,
$$


возникающую в теории лазеров [5]. Здесь $0<\varepsilon \ll 1$, положительные параметры $\alpha, \beta, \gamma$ имеют порядок единицы и, кроме того, выполняется условие

$$
\alpha>\beta+1
$$

Для отыскания циклов системы (1.1) зафиксируем произвольно $x_{0} \in[\beta+1, \alpha]$ и обозначим через

$$
\Gamma(\varepsilon)=\left\{(x, y): x=x\left(t, x_{0}, \varepsilon\right), y=y\left(t, x_{0}, \varepsilon\right), t \geqslant 0\right\}
$$

ее траекторию с начальными условиями $x\left(0, x_{0}, \varepsilon\right)=x_{0}, y\left(0, x_{0}, \varepsilon\right)=1$. Далее, рассмотрим второй положительный корень $t=T\left(x_{0}, \varepsilon\right)$ уравнения $y\left(t, x_{0}, \varepsilon\right)=1$ (если он существует) и определим оператор последования Пуанкаре посредством равенства

$$
\left.x_{0} \rightarrow \Pi\left(x_{0}, \varepsilon\right) \stackrel{\text { def }}{=} x\left(t, x_{0}, \varepsilon\right)\right|_{t=T\left(x_{0}, \varepsilon\right)} .
$$

Наша ближайшая задача - выяснить асимптотическое поведение при $\varepsilon \rightarrow 0$ оператора (1.4).

Перед формулировкой строгих утверждений изложим ряд соображений эвристического характера. С этой целью обратим внимание, что в силу выбора $x_{0}$ и условия (1.2) справедливо неравенство

$$
x_{0}-1-\frac{\beta}{1+\gamma y}>0 \quad \forall y \geqslant 0 .
$$

А отсюда и из (1.1) заключаем, что сначала движение фазовой точки $(x, y)$ происходит в асимптотически малой окрестности луча $\left\{(x, y): x=x_{0}, y \geqslant 1\right\}$, причем компонента $y\left(t, x_{0}, \varepsilon\right)$ за асимптотически малое время (порядка $\varepsilon \ln (1 / \varepsilon)$ ) достигает значения $y=\varepsilon^{-3 / 4}$. Соответствующий участок траектории $\Gamma(\varepsilon)$ обозначим через $\Gamma_{1}(\varepsilon)$ (см. рис. 1$)$ и назовем участком взлета.

Следующий участок, лежащий в полуплоскости $y \geqslant \varepsilon^{-3 / 4}$, обозначим через $\Gamma_{2}(\varepsilon)$ и будем называть участком поворота. Для выявления его асимптотического поведения сделаем в (1.1) замену $u=\varepsilon y$ и возьмем $x$ за новое время. В результате после отбрасывания асимптотически малых добавок для отыскания $u=u(x)$ получаем задачу Коши

$$
\frac{d u}{d x}=-\frac{x-1}{x},\left.\quad u\right|_{x=x_{0}}=0 .
$$

Нетрудно увидеть, что решение задачи (1.6) задается равенством

$$
u(x)=-\left(x-x_{0}\right)+\ln \frac{x}{x_{0}}, \quad 0<x \leqslant x_{0} .
$$

Далее, поскольку $u\left(x_{0}\right)=0, u^{\prime}(x)<0$ при $1<x \leqslant x_{0}, u^{\prime}(x)>0$ при $0<x<1$, $u(x) \rightarrow-\infty$ при $x \rightarrow+0$, то на интервале $(0,1)$ уравнение $u(x)=0$ допускает единственное решение $x=x_{1}$, причем $u(x)>0$ при $x_{1}<x<x_{0}$. А это значит, что, двигаясь по кривой $\Gamma_{2}(\varepsilon)$, фазовая точка системы (1.1) сначала покидает прямую $y=\varepsilon^{-3 / 4}$, а затем снова возвращается на нее, т.е. совершает поворот 


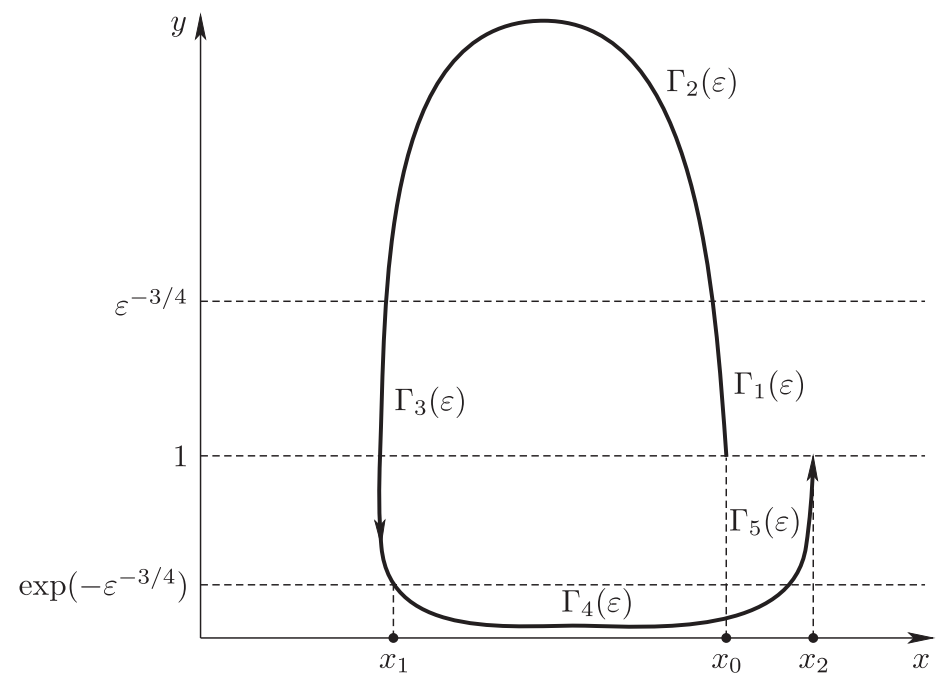

Рис. 1

(см. рис. 1). Более того, после перехода к переменным $(x, u)$ интересующий нас участок $\Gamma_{2}(\varepsilon) \subset \Gamma(\varepsilon)$ имеет своим пределом при $\varepsilon \rightarrow 0$ кривую

$$
\Gamma_{2}^{0}=\left\{(x, u): u=u(x), x_{1} \leqslant x \leqslant x_{0}\right\} .
$$

Вид этой кривой представлен на рис. 2.

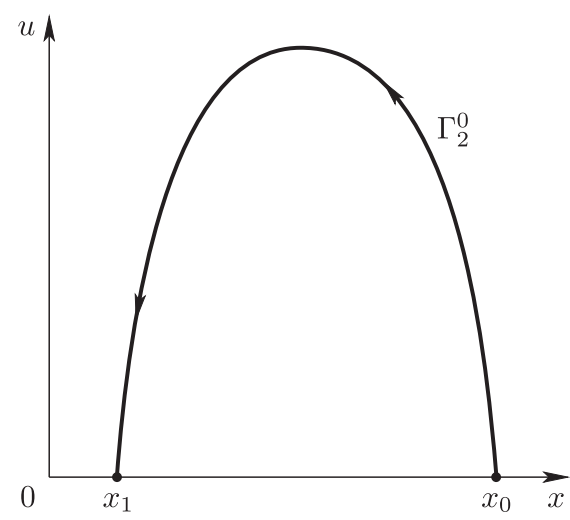

Рис. 2

Добавим еще, что время движения по участку $\Gamma_{2}(\varepsilon)$ асимптотически мало, а точнее говоря, является величиной порядка $\varepsilon \ln (1 / \varepsilon)$. Любой же кусок кривой $\Gamma_{2}(\varepsilon)$, отвечающий значениям $x \in[a, b] \subset\left(x_{1}, x_{0}\right)$, где $a, b=$ const $>0$, фазовая точка $(x, y)$ проходит за время порядка $\varepsilon$. 
Следующий участок $\Gamma_{3}(\varepsilon)$ кривой $\Gamma(\varepsilon)$, соответствующий значениям переменной $y$ из отрезка $\exp \left(-\varepsilon^{-3 / 4}\right) \leqslant y \leqslant \varepsilon^{-3 / 4}$, аналогичен уже рассмотренному случаю $\Gamma_{1}(\varepsilon)$. Действительно, так как $x_{1}-1-\beta /(1+\gamma y)<0$ для всех $y \geqslant 0$, то кривая $\Gamma_{3}(\varepsilon)$ асимптотически близка к отрезку

$$
\left\{(x, y): x=x_{1}, \exp \left(-\varepsilon^{-3 / 4}\right) \leqslant y \leqslant \varepsilon^{-3 / 4}\right\}
$$

(см. рис. 1). Однако поскольку здесь переменная у убывает, то в отличие от $\Gamma_{1}(\varepsilon)$ данный фрагмент кривой $\Gamma(\varepsilon)$ уместно назвать участком падения. Что же касается времени "падения", то оно имеет порядок $\varepsilon^{1 / 4}$.

Очередной участок $\Gamma_{4}(\varepsilon)$, лежащий в полуплоскости $y \leqslant \exp \left(-\varepsilon^{-3 / 4}\right)$ (см. рис. 1), назовем участком медленного движения. Для его описания перейдем в системе (1.1) к новой переменной $v=\varepsilon \ln y$ и возьмем $x$ за новое время. В результате для нахождения $v=v(x)$ после отбрасывания асимптотически малых слагаемых приходим к задаче Коши

$$
\frac{d v}{d x}=\frac{x-1-\beta}{\alpha-x},\left.\quad v\right|_{x=x_{1}}=0,
$$

из которой, в свою очередь, выводим

$$
v(x)=-\left(x-x_{1}\right)-(\alpha-1-\beta) \ln \frac{\alpha-x}{\alpha-x_{1}}, \quad x_{1} \leqslant x<\alpha .
$$

Остановимся на некоторых свойствах функции (1.9). Несложная проверка показывает, что

$$
\begin{gathered}
v^{\prime}(x)<0 \quad \text { при } x_{1} \leqslant x<\beta+1, \quad v^{\prime}(x)>0 \quad \text { при } \beta+1<x<\alpha, \\
v(x) \rightarrow+\infty \quad \text { при } \quad x \rightarrow \alpha-0 .
\end{gathered}
$$

Следовательно, у уравнения $v(x)=0$ на интервале $\beta+1<x<\alpha$ существует единственное решение $x=x_{2}$, причем $v(x)<0$ при $x_{1}<x<x_{2}$.

Перечисленные свойства $v(x)$ позволяют утверждать, что график кривой $\Gamma_{4}(\varepsilon)$ действительно имеет вид, показанный на рис. 1. Кроме того, как и в случае $\Gamma_{2}(\varepsilon)$, после перехода к переменным $(x, v)$ эта кривая стремится при $\varepsilon \rightarrow 0$ к пределу

$$
\Gamma_{4}^{0}=\left\{(x, v): v=v(x), x_{1} \leqslant x \leqslant x_{2}\right\}
$$

(см. рис. 3).

Отдельного упоминания заслуживает вопрос о времени движения по кривой $\Gamma_{4}(\varepsilon)$. В отличие от всех предыдущих участков здесь оно асимптотически конечно, т.е. допускает при $\varepsilon \rightarrow 0$ конечный предел

$$
T_{0}=\ln \frac{\alpha-x_{1}}{\alpha-x_{2}}
$$

Таким образом, прояснилась причина, по которой данный участок был назван участком медленного движения. 


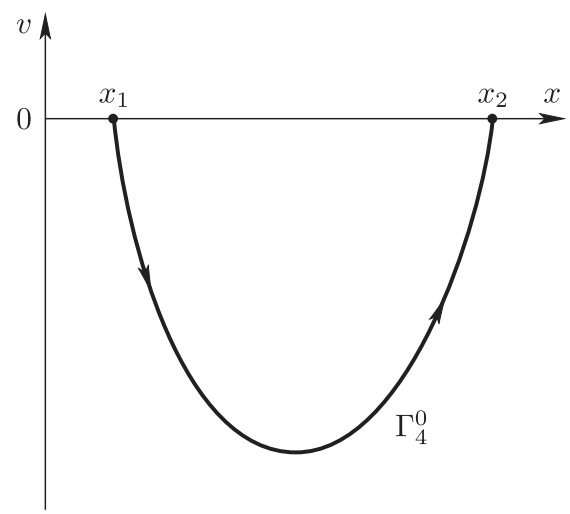

Рис. 3

На заключительном этапе обратимся к так называемому участку подъема $\Gamma_{5}(\varepsilon) \subset \Gamma(\varepsilon)$, расположенному в полосе $\exp \left(-\varepsilon^{-3 / 4}\right) \leqslant y \leqslant 1$ (см. рис. 1$)$. Заметим, что поскольку в данном случае справедливо неравенство, получающееся из (1.5) при замене $x_{0}$ на $x_{2}$, то кривая $\Gamma_{5}(\varepsilon)$ асимптотически близка к отрезку $\left\{(x, y): x=x_{2}, \exp \left(-\varepsilon^{-3 / 4}\right) \leqslant y \leqslant 1\right\}$, причем при движении по $\Gamma_{5}(\varepsilon)$ переменная $y$ возрастает (отсюда и название - участок подъема). Добавим еще, что время "подъема" асимптотически мало (имеет порядок $\varepsilon^{1 / 4}$ ).

Строгое обоснование всех перечисленных фактов, касающихся асимптотического поведения траектории (1.3), базируется на общей теории неклассических релаксационных колебаний, развитой в [2]-[4]. Из основных результатов этой теории вытекает, в частности, следующее утверждение.

Лемма 1.1. При всех достаточно мальх $\varepsilon>0$ оператор (1.4) определен на отрезке $\beta+1 \leqslant x_{0} \leqslant \alpha$ и удовлетворяет пределъным равенствам

$$
\begin{aligned}
& \lim _{\varepsilon \rightarrow 0} \max _{\beta+1 \leqslant x_{0} \leqslant \alpha}\left|\Pi\left(x_{0}, \varepsilon\right)-\Pi\left(x_{0}\right)\right|=0, \\
& \lim _{\varepsilon \rightarrow 0} \max _{\beta+1 \leqslant x_{0} \leqslant \alpha}\left|\Pi^{\prime}\left(x_{0}, \varepsilon\right)-\Pi^{\prime}\left(x_{0}\right)\right|=0 .
\end{aligned}
$$

Здесъ штрих - производная по $x_{0}$, а оператор $\Pi\left(x_{0}\right)$ задается соотношениями

$$
\Pi=\Pi_{2} \circ \Pi_{1}, \quad \Pi_{1}: x_{0} \rightarrow x_{1}=x_{1}\left(x_{0}\right), \quad \Pi_{2}: x_{1} \rightarrow x_{2}=x_{2}\left(x_{1}\right),
$$

где $x_{1}$ и $x_{2}$ - введенные выше корни уравнений $u(x)=0$ u $v(x)=0$ соответственно.

Итак, вопрос о существовании и устойчивости циклов системы (1.1) сводится к аналогичному вопросу для неподвижных точек предельного оператора (1.12). В связи с этим становится актуальной следующая

Лемма 1.2. Отображение (1.12) имеет на отрезке $\beta+1 \leqslant x_{0} \leqslant \alpha$ единственную экспоненциально устойчивую неподвижную точку $x_{0}=x_{0}^{*}$. 
ДокАЗАтЕльство. Заметим прежде всего, что отображение (1.12) преобразует отрезок $\beta+1 \leqslant x_{0} \leqslant \alpha$ строго в себя и, следовательно, имеет на интервале $\beta+1<x_{0}<\alpha$ хотя бы одну неподвижную точку $x_{0}=x_{0}^{*}$. Кроме того, из включений $x_{0} \in[\beta+1, \alpha], x_{1} \in(0,1), x_{2} \in(\beta+1, \alpha)$ и формул (1.6)-(1.9) вытекает, что

$$
\Pi^{\prime}\left(x_{0}\right)=\frac{x_{0}-1}{x_{0}} \cdot \frac{x_{1}}{1-x_{1}} \cdot \frac{\alpha-x_{2}}{\alpha-x_{1}} \cdot \frac{1+\beta-x_{1}}{x_{2}-1-\beta}>0 \quad \forall x_{0} \in[\beta+1, \alpha] .
$$

Таким образом, для обоснования леммы достаточно убедиться, что неподвижная точка $x_{0}^{*}$ единственна и удовлетворяет неравенству

$$
\Pi^{\prime}\left(x_{0}^{*}\right)<1
$$

Отыскание возможных неподвижных точек отображения П $\left(x_{0}\right)$ проводится по следующей схеме. Сначала обратимся к соотношениям $u\left(x_{1}\right)=0, v\left(x_{2}\right)=0$ для нахождения функций $x_{1}=x_{1}\left(x_{0}\right), x_{2}=x_{2}\left(x_{1}\right)$ и положим во втором из них $x_{2}=x_{0}$. В результате, опираясь на формулы (1.7), (1.9), для определения неизвестных $x_{0}, x_{1}$ получим систему уравнений

$$
x_{1}-x_{0}=\ln \frac{x_{1}}{x_{0}}, \quad x_{0}-x_{1}=(\alpha-1-\beta) \ln \frac{\alpha-x_{1}}{\alpha-x_{0}} .
$$

Как оказывается, систему (1.15) можно свести к одному скалярному уравнению. Действительно, введем в рассмотрение переменную

$$
z=\frac{\alpha-x_{1}}{\alpha-x_{0}}>1
$$

и попытаемся выразить $x_{0}, x_{1}$ через $z$. На этом пути последовательно убеждаемся, что

$$
x_{0}=\frac{\alpha(z-1) z^{\alpha-\beta-1}}{z^{\alpha-\beta}-1}, \quad x_{1}=\frac{\alpha(z-1)}{z^{\alpha-\beta}-1},
$$

а сама переменная (1.16) удовлетворяет уравнению

$$
P(z) \stackrel{\text { def }}{=}(\alpha-\beta-1)\left(z^{\alpha-\beta}-1\right) \ln z+\alpha(z-1)\left(1-z^{\alpha-\beta-1}\right)=0 .
$$

Итак, мы показали, что если $x_{0}=x_{0}^{*}-$ неподвижная точка отображения (1.12), то величина $z_{*}$, вычисленная по формуле (1.16) при $x_{0}=x_{0}^{*}, x_{1}=x_{1}^{*}$, где $x_{1}^{*}=\Pi_{1}\left(x_{0}^{*}\right)$, является корнем уравнения $(1.18)$, лежащим на полуоси $z>1$. Тем самым, для доказательства единственности неподвижной точки $x_{0}^{*}$ остается убедиться в единственности такого корня.

Вопрос о количестве корней уравнения (1.18) изучим сначала на полуоси $z>0$. Непосредственная проверка показывает, что

$$
\begin{gathered}
P(1)=P^{\prime}(1)=0, \quad P^{\prime \prime}(1)=-2 \beta(\alpha-\beta-1)<0, \\
\lim _{z \rightarrow+0} P(z)=\lim _{z \rightarrow+\infty} P(z)=+\infty .
\end{gathered}
$$


Из свойств (1.19), в свою очередь, следует, что общее количество нулей функции $P(z)$ четно и помимо двукратного корня $z=1$ на множествах $0<z<1$ и $z>1$ имеется еще по крайней мере по одному из них.

Заметим далее, что $\Phi(t)=P(\exp t), t \in \mathbb{R}$, является линейной комбинацией функций

$$
\exp (\alpha-\beta) t, \quad t \exp (\alpha-\beta) t, \quad 1, \quad t, \quad \exp t, \quad \exp (\alpha-\beta-1) t .
$$

Тем самым, $\Phi(t)$ удовлетворяет некоторому линейному дифференциальному уравнению шестого порядка с постоянными коэффициентами и с вещественными корнями характеристического полинома, а значит, допускает на всей оси $t \in \mathbb{R}$ не более пяти корней. Но поскольку, напомним, общее число всех корней должно быть четным, то на самом деле их ровно четыре. В частности, на интересующей нас полуоси $z>1$ лежит единственный корень $z=z_{*}$, причем в силу (1.19) с необходимостью

$$
P^{\prime}\left(z_{*}\right)>0
$$

На завершающем этапе доказательства леммы установим неравенство (1.14). С этой целью подставим соотношение $x_{2}=x_{0}$ вместе с формулами (1.17) при $z=z_{*}$ в (1.13). В результате после некоторых преобразований и сокращения на $z_{*}^{\alpha-\beta}-1>0$ приходим к выводу, что интересующее нас свойство (1.14) эквивалентно оценке

$$
\alpha\left(z_{*}-1\right)\left(z_{*}^{2(\alpha-\beta)-1}-1\right)-\alpha(\alpha-\beta-1)\left(z_{*}-1\right)^{2} z_{*}^{\alpha-\beta-1}-(1+\beta)\left(z_{*}^{\alpha-\beta}-1\right)^{2}>0 .
$$

Для проверки требования (1.21) обратимся к уже известному соотношению (1.20), подставим в него формулы

$$
\begin{gathered}
P^{\prime}\left(z_{*}\right)=(\alpha-\beta-1)(\alpha-\beta) z_{*}^{\alpha-\beta-1} \ln z_{*}+(\alpha-\beta-1)\left(z_{*}^{\alpha-\beta}-1\right) \frac{1}{z_{*}} \\
+\alpha\left(1-z_{*}^{\alpha-\beta-1}\right)-\alpha\left(z_{*}-1\right)(\alpha-\beta-1) z_{*}^{\alpha-\beta-2} \\
(\alpha-\beta-1) \ln z_{*}=\frac{\alpha\left(z_{*}-1\right)\left(z_{*}^{\alpha-\beta-1}-1\right)}{z_{*}^{\alpha-\beta}-1}
\end{gathered}
$$

и умножим получившееся неравенство на $z_{*}\left(z_{*}^{\alpha-\beta}-1\right)$. В итоге, как нетрудно убедиться, оно преобразуется к виду (1.21). Лемма 1.2 доказана.

Проделанный анализ позволяет разобраться с интересующим нас вопросом о периодических движениях системы (1.1). Действительно, из предельных равенств (1.11) вытекает, что отображение последования Пуанкаре (1.4) при всех достаточно малых $\varepsilon>0$ допускает единственную экспоненциально устойчивую неподвижную точку $x_{0}=x_{0}^{*}(\varepsilon)$, асимптотически близкую к $x_{0}^{*}$. В системе (1.1) указанной неподвижной точке отвечает устойчивый цикл $\left(x_{*}(t, \varepsilon), y_{*}(t, \varepsilon)\right)$, $y_{*}(0, \varepsilon) \equiv 1$ периода $T_{*}(\varepsilon)$ со следующими свойствами.

Во-первых, справедливо соотношение

$$
\lim _{\varepsilon \rightarrow 0} T_{*}(\varepsilon)=T_{*},
$$

где $T_{*}$ - величина, получающаяся из (1.10) при $x_{0}=x_{0}^{*}, x_{1}=x_{1}^{*}$. 
Во-вторых, компонента $y_{*}(t, \varepsilon)$ при $\varepsilon \rightarrow 0$ ведет себя $\delta$-образно. А именно, на промежутке $0 \leqslant t \leqslant t_{*}(\varepsilon)$, где $t_{*}(\varepsilon)=O(\varepsilon \ln (1 / \varepsilon))$ - первый положительный корень уравнения $y_{*}(t, \varepsilon)=1$, происходит асимптотически высокий (порядка $\varepsilon^{-1}$ ) всплеск, причем

$$
\lim _{\varepsilon \rightarrow 0} \int_{0}^{t_{*}(\varepsilon)} y_{*}(t, \varepsilon) d t=\ln \frac{x_{0}^{*}}{x_{1}^{*}}>0 .
$$

Все же остальное время функция $y_{*}(t, \varepsilon)$ экспоненциально мала. Точнее говоря, для любых $\delta_{1}, \delta_{2} \in\left(0, T_{*} / 2\right)$ на отрезке $\delta_{1} \leqslant t \leqslant T_{*}(\varepsilon)-\delta_{2}$ выполняется оценка вида

$$
y_{*}(t, \varepsilon) \leqslant M_{1} \exp \left(-\frac{M_{2}}{\varepsilon}\right), \quad M_{1}, M_{2}=\text { const }>0 .
$$

В третьих, компонента $x_{*}(t, \varepsilon)$ при $\varepsilon \rightarrow 0$ сходится равномерно по $t \in\left[\delta_{1}\right.$, $\left.T_{*}(\varepsilon)-\delta_{2}\right]$, где, как и выше, $\delta_{1}, \delta_{2}=$ const $\in\left(0, T_{*} / 2\right)$, к функции $x_{*}(t)$, являющейся решением задачи Коши $\dot{x}=\alpha-x,\left.x\right|_{t=0}=x_{1}^{*}$. Подчеркнем, что, будучи продолженной на всю ось $t$ по закону периодичности с периодом $T_{*}$, функция $x_{*}(t)$ оказывается разрывной в точках $t=k T_{*}, k \in \mathbb{Z}$.

В дальнейшем релаксационный цикл с перечисленными свойствами будем называть неклассическим или циклом импульсного типа. Наглядное представление о нем дает рис. 4, на котором приведены графики компонент этого цикла при значениях параметров $\alpha=4, \beta=1, \gamma=1, \varepsilon=0.01$ (сплошной линией показан график $y_{*}(t+c, \varepsilon)$, а пунктирной - график $x_{*}(t+c, \varepsilon)$, где $c \in \mathbb{R}-$ некоторый фазовый сдвиг).

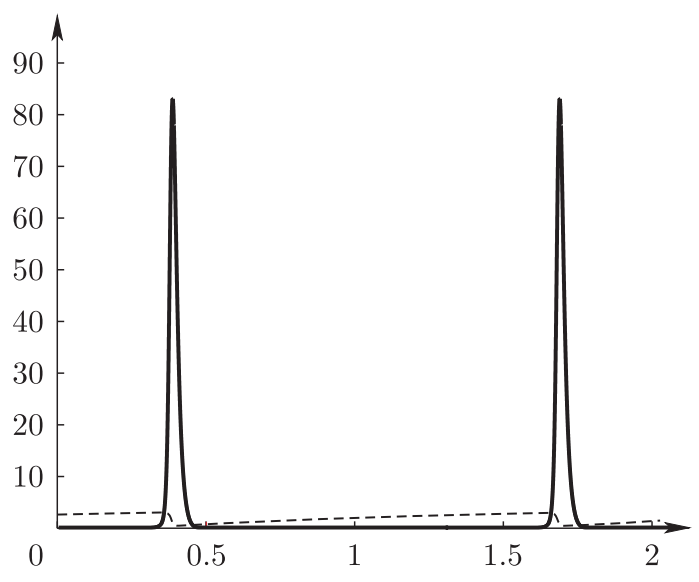

Рис. 4

В настоящей статье теория неклассических релаксационных колебаний распространяется на системы с запаздыванием вида

$$
\dot{x}=f(x, y), \quad \varepsilon \dot{y}=[g(x, y)-y(t-h)] y,
$$


где $0<\varepsilon \ll 1, h=\mathrm{const}>0 ; f, g \in C^{\infty}(K), K=\left\{(x, y): x>-\delta_{0}, y>-\delta_{0}\right\}$, $\delta_{0}>0$. Точнее говоря, в дальнейшем будет установлено, что при некоторых дополнительных ограничениях на функции $f, g$ и запаздывание $h$ в системе $(1.25)$ существует устойчивый релаксационный цикл, координаты $x_{*}(t, \varepsilon), y_{*}(t, \varepsilon)$ которого и период $T_{*}(\varepsilon)$ обладают свойствами, аналогичными перечисленным выше (см. (1.22)-(1.24)).

\section{§ 2. Постановка задачи и основной результат}

2.1. Описание рассматриваемого класса уравнений. Поскольку нас интересуют релаксационные циклы системы (1.25) с $\delta$-образной компонентой $y$, то в первую очередь сформулируем серию ограничений, касающихся поведения функций $f, g$ при больших значениях $y$ и при $y=0$.

Условие 2.1. При $y \rightarrow+\infty$ равномерно по $x$ из любого замкнутого и ограниченного подмножества полуоси $x \geqslant 0$ справедливы асимптотические разложения

$$
\begin{gathered}
f(x, y)=y\left[a_{0}(x)+\frac{a_{1}(x)}{y}+\frac{a_{2}(x)}{y^{2}}+\cdots\right], \\
g(x, y)=b_{0}(x)+\frac{b_{1}(x)}{y}+\frac{b_{2}(x)}{y^{2}}+\cdots,
\end{gathered}
$$

сохраняющие силу при дифференцировании по $x, y$ в любом порядке и любое число раз.

Условие 2.2. Фигурирующие в $(2.1)$ коэффициенты $a_{0}(x), b_{0}(x)$ удовлетворяют неравенствам

$$
\begin{gathered}
a_{0}(x)>0 \quad \forall x>0, \\
b_{0}(x)>0 \quad \text { при } x \in\left(0, q_{1}\right), \quad b_{0}(x)<0 \quad \text { при } \quad x>q_{1},
\end{gathered}
$$

где $q_{1}$ - некоторая положительная постоянная.

Условие 2.3. Считаем, что

$$
\begin{gathered}
f(x, 0)<0 \quad \forall x>0, \quad f(0,0)=0, \\
g(x, 0)>0 \quad \text { при } 0 \leqslant x<q_{2}, \quad g(x, 0)<0 \quad \text { при } x>q_{2},
\end{gathered}
$$

где $q_{2}=$ const $>0$.

Интересно отметить, что приведенные условия допускают наглядную биологическую интерпретацию. Действительно, если предположить, что $x, y-$ плотности численностей популяций хищника и жертвы соответственно, то тогда требованиям (2.2)-(2.5) можно придать следующий смысл.

Первое из них, а именно неравенство (2.2), означает, что при больших $y$ (т.е. когда пищи много) численность хищника $x$ должна расти. Интерпретация ограничений $(2.3),(2.5)$ также не вызывает затруднений. Оба они свидетельствуют о том, что в случае, когда численность хищника $x$ меньше некоторого 
порогового значения, скорость изменения численности жертвы положительна, а когда больше - отрицательна. Добавим еще, что при $y \gg 1$ и $y=0$ соответствующие пороговые значения $x=q_{1}$ и $x=q_{2}$, вообще говоря, различны.

Смысл оставшихся требований (2.4) очевиден: в случае $y=0$ имеем $x(t) \rightarrow 0$, $t \rightarrow+\infty$, т.е. при отсутствии жертвы хищник вымирает. Биологическими причинами продиктован и характер зависимости второго уравнения системы (1.25) от $y(t-h)$. Такого рода зависимость уместно назвать хатчинсоновской, так как при фиксированном $x$ это уравнение представляет собой некоторую модификацию известного уравнения Хатчинсона (см. [6])

$$
\varepsilon \dot{y}=(1-y(t-h)) y,
$$

описывающего колебания плотности численности млекопитающих.

Для формулировки следующей серии ограничений нам потребуются функции

$$
\gamma_{0}\left(x, x_{0}\right)=\int_{x_{0}}^{x} \frac{b_{0}(s)}{a_{0}(s)} d s, \quad \gamma_{1}\left(x, x_{1}\right)=\int_{x_{1}}^{x} \frac{g(s, 0)}{f(s, 0)} d s .
$$

УсловиЕ 2.4. Найдется такое $x_{0}=x_{0}^{*} \in\left(0, \min \left(q_{1}, q_{2}\right)\right)$, что уравнение

$$
\gamma_{0}\left(x, x_{0}\right)=0
$$

при $x_{0}=x_{0}^{*}$ допускает решение $x=x_{1}^{*}>\max \left(q_{1}, q_{2}\right)$.

Приведенное условие позволяет определить некоторое одномерное отображение, аналогичное рассмотренному в предыдущем параграфе (см. (1.12)). Построение этого отображения проведем в два этапа.

На первом этапе применим к уравнению $(2.8)$ в точке $\left(x, x_{0}\right)=\left(x_{1}^{*}, x_{0}^{*}\right)$ теорему о неявной функции по переменной $x$. Подчеркнем, что условия этой теоремы здесь заведомо выполняются, поскольку $\gamma_{0}\left(x_{1}^{*}, x_{0}^{*}\right)=0$ и в силу вытекающих из $(2.2),(2.3),(2.7)$ свойств

$$
\frac{\partial \gamma_{0}}{\partial x}\left(x, x_{0}\right)>0 \quad \text { при } x \in\left(0, q_{1}\right), \quad \frac{\partial \gamma_{0}}{\partial x}\left(x, x_{0}\right)<0 \quad \text { при } \quad x>q_{1}
$$

справедливо неравенство

$$
\left.\frac{\partial \gamma_{0}}{\partial x}\right|_{\left(x, x_{0}\right)=\left(x_{1}^{*}, x_{0}^{*}\right)}<0
$$

Таким образом, из (2.8) однозначно определяется функция

$$
x=x_{1}\left(x_{0}\right), \quad x_{1}\left(x_{0}^{*}\right)=x_{1}^{*},
$$

причем

$$
x_{1}\left(x_{0}\right)>\max \left(q_{1}, q_{2}\right) \quad \forall x_{0} \in[a, b] .
$$

Здесь $[a, b] \subset\left(0, \min \left(q_{1}, q_{2}\right)\right)$ - некоторый отрезок достаточно малой длины, имеющий $x_{0}^{*}$ своей внутренней точкой. 
На втором этапе обратимся к уравнению

$$
\gamma_{1}\left(x, x_{1}\right)=\int_{x_{0}}^{x_{1}} \frac{d s}{a_{0}(s)},
$$

считая, что $x_{0}$ пробегает фигурирующий в (2.10) отрезок $[a, b]$, а $x_{1}=x_{1}\left(x_{0}\right)$ функция из (2.10). Привлекая, далее, соотношения (2.4), (2.5), (2.7), нетрудно увидеть, что

$$
\begin{gathered}
\frac{\partial \gamma_{1}}{\partial x}\left(x, x_{1}\right)>0 \quad \text { при } x>q_{2}, \quad \frac{\partial \gamma_{1}}{\partial x}\left(x, x_{1}\right)<0 \quad \text { при } x \in\left(0, q_{2}\right), \\
\gamma_{1}\left(x, x_{1}\right) \rightarrow+\infty \quad \text { при } x \rightarrow+0 .
\end{gathered}
$$

А отсюда с учетом вытекающего из (2.2) неравенства

$$
\int_{x_{0}}^{x_{1}} \frac{d s}{a_{0}(s)}>0
$$

и оценки $x_{1}>q_{2}$ (см. (2.10)) заключаем, что уравнение $(2.11)$ имеет на интервале $0<x<q_{2}$ единственное решение $x=x_{2}\left(x_{0}\right)$. Тем самым, на отрезке $a \leqslant x_{0} \leqslant b$ корректно определено одномерное отображение

$$
x_{0} \rightarrow \psi\left(x_{0}\right) \stackrel{\text { def }}{=} x_{2}\left(x_{0}\right)
$$

причем, как нетрудно проверить (см. аналогичное место в случае отображения $(1.12))$, справедливы свойства $\psi^{\prime}\left(x_{0}\right)>0, x_{1}^{\prime}\left(x_{0}\right)<0$ для всех $x_{0} \in[a, b]$.

УСловие 2.5. Предполагаем, что значение $x_{0}=x_{0}^{*}$ из условия 2.4 является экспоненциально устойчивой неподвижной точкой отображения (2.14), т.е.

$$
\psi\left(x_{0}^{*}\right)=x_{0}^{*}, \quad \psi^{\prime}\left(x_{0}^{*}\right)<1 .
$$

Сформулируем теперь ограничение на величину запаздывания $h$ из (1.25). С этой целью обратимся к аналогичному (2.11) уравнению

$$
\gamma_{1}\left(x, x_{1}\right)=0
$$

при $x_{1}=x_{1}\left(x_{0}\right), x_{0} \in[a, b]$. Из отмеченных выше свойств $(2.12),(2.13)$ вытекает, что уравнение (2.16) имеет на интервале $0<x<q_{2}$ единственное решение $x=x_{3}\left(x_{0}\right)$ такое, что $x_{3}\left(x_{0}\right)>x_{2}\left(x_{0}\right) \forall x_{0} \in[a, b]$.

Условие 2.6. Считаем, что

$$
h<h_{*} \stackrel{\text { def }}{=}-\int_{x_{3}^{*}}^{x_{1}^{*}} \frac{d s}{f(s, 0)},
$$

где $x_{3}^{*}=x_{3}\left(x_{0}^{*}\right)$.

Обратим внимание, что все перечисленные ограничения на $f, g$ относятся к случаям $y \gg 1$ и $y=0$. Что же касается заключительного условия, то оно представляет собой некое связующее звено между большими и малыми значениями $y$. 
УСловие 2.7. В точках $x=x_{0}^{*}$ и $x=x_{1}^{*}$, о которых говорится в условии 2.4 , имеют место неравенства

$$
g\left(x_{0}^{*}, y\right)>0, \quad g\left(x_{1}^{*}, y\right)<0 \quad \forall y \geqslant 0 .
$$

Подчеркнем, что в силу соотношений $(2.1),(2.3),(2.5)$ оценки (2.18) заведомо справедливы при $y \ll 1$ и $y \gg 1$. При остальных же значениях $y$ их выполнения приходится требовать.

2.2. Основной результат. Перейдем к вопросу о существовании у системы (1.25) экспоненциально орбитально устойчивого (в метрике фазового пространства $\mathbb{R} \times C[-h, 0])$ релаксационного цикла импульсного типа. Перед формулировкой соответствующего строгого утверждения введем в рассмотрение величины

$$
T_{*}=-\int_{x_{0}^{*}}^{x_{1}^{*}} \frac{d s}{f(s, 0)}>h_{*}, \quad y_{\mathrm{int}}^{*}=\int_{x_{0}^{*}}^{x_{1}^{*}} \frac{d s}{a_{0}(s)}>0, \quad y_{\max }^{*}=\gamma_{0}\left(q_{1}, x_{0}^{*}\right)>0
$$

и функцию $x_{*}(t)$, являющуюся решением задачи Коши

$$
\dot{x}=f(x, 0),\left.\quad x\right|_{t=0}=x_{1}^{*} .
$$

ТеОрема 2.1. Пусть выполнены условия 2.1-2.7. Тогда найдется такое достаточно малое $\varepsilon_{0}>0$, что при всех $0<\varepsilon \leqslant \varepsilon_{0}$ система (1.25) имеет экспоненциально орбитально устойчивый релаксационный цикл $\left(x_{*}(t, \varepsilon), y_{*}(t, \varepsilon)\right)$ периода $T_{*}(\varepsilon)$, причем $y_{*}(t, \varepsilon)>0, y_{*}(0, \varepsilon) \equiv 1$. Для этого иикла справедливы предельные равенства

$$
\begin{gathered}
\lim _{\varepsilon \rightarrow 0} T_{*}(\varepsilon)=T_{*}, \\
\lim _{\varepsilon \rightarrow 0} \int_{0}^{t_{*}(\varepsilon)} y_{*}(t, \varepsilon) d t=y_{\text {int }}^{*}, \quad \lim _{\varepsilon \rightarrow 0} \max _{t}\left(\varepsilon y_{*}(t, \varepsilon)\right)=y_{\max }^{*}, \\
\lim _{\varepsilon \rightarrow 0} \max _{\delta_{1} \leqslant t \leqslant T_{*}(\varepsilon)-\delta_{2}}\left|x_{*}(t, \varepsilon)-x_{*}(t)\right|=0
\end{gathered}
$$

и оценка вида

$$
\max _{\delta_{1} \leqslant t \leqslant T_{*}(\varepsilon)-\delta_{2}} y_{*}(t, \varepsilon) \leqslant M_{1} \exp \left(-\frac{M_{2}}{\varepsilon}\right), \quad M_{1}, M_{2}=\text { const }>0 .
$$

Здесъ момент времени $t_{*}(\varepsilon)=O(\varepsilon \ln (1 / \varepsilon))$ - первый положительный корень уравнения $y_{*}(t, \varepsilon)=1$, а постоянные $\delta_{1}, \delta_{2} \in\left(0, T_{*} / 2\right)$ произвольно фиксированы.

Доказательство сформулированной теоремы, приводящееся в следующих двух параграфах, опирается на некоторые дополнительные конструкции. Для их описания введем в рассмотрение замкнутое ограниченное выпуклое множество $S \subset \mathbb{R} \times C[-h, 0]$, состоящее из таких пар $\left(x_{0}, \varphi(t)\right)$, что

$$
m_{2} t \leqslant \varphi(t) \leqslant m_{1} t \quad \forall t \in[-h, 0], \quad a \leqslant x_{0} \leqslant b .
$$


Здесь $[a, b]$ - отрезок, фигурирующий в (2.10), а универсальные (не зависящие от $\varphi(t))$ постоянные $m_{2}>m_{1}>0$ пока произвольны. Далее, для каждого элемента $\left(x_{0}, \varphi\right) \in S$ рассмотрим решение $(x, y)=\left(x_{\left(x_{0}, \varphi\right)}(t, \varepsilon), y_{\left(x_{0}, \varphi\right)}(t, \varepsilon)\right), t \geqslant 0$, системы (1.25) с начальными условиями

$$
\left.x\right|_{t=0}=x_{0}, \quad y=\exp \frac{\varphi(t)}{\varepsilon} \quad \text { при } \quad-h \leqslant t \leqslant 0,
$$

а через $t_{\left(x_{0}, \varphi\right)}^{(1)}(\varepsilon)$ и $t_{\left(x_{0}, \varphi\right)}^{(2)}(\varepsilon)$ обозначим первый и второй положительные корни уравнения

$$
y_{\left(x_{0}, \varphi\right)}(t, \varepsilon)=1
$$

соответственно (если они существуют). И, наконец, зададим оператор последования Пуанкаре $\Pi_{\varepsilon}: S \rightarrow \mathbb{R} \times C[-h, 0]$ с помощью равенства

$$
\begin{gathered}
\Pi_{\varepsilon}\left[\left(x_{0}, \varphi\right)\right]=\left(x_{\left(x_{0}, \varphi\right)}\left(t_{\left(x_{0}, \varphi\right)}^{(2)}(\varepsilon), \varepsilon\right), \varepsilon \ln y_{\left(x_{0}, \varphi\right)}\left(t+t_{\left(x_{0}, \varphi\right)}^{(2)}(\varepsilon), \varepsilon\right)\right), \\
-h \leqslant t \leqslant 0 .
\end{gathered}
$$

Последующий план действий таков. Сначала мы уточним выбор отрезка $[a, b]$ из $(2.25)$ и получим равномерные по $\left(x_{0}, \varphi\right) \in S$ асимптотические формулы для компонент $x_{\left(x_{0}, \varphi\right)}(t, \varepsilon), y_{\left(x_{0}, \varphi\right)}(t, \varepsilon)$ решения задачи Коши $(1.25),(2.26)$ на различных промежутках изменения $t$. Затем покажем, что при подходящем выборе параметров $m_{1}, m_{2}$ оператор (2.28) преобразует множество $S$ в себя. После этого убедимся, что интересующее нас периодическое решение $\left(x_{*}(t, \varepsilon), y_{*}(t, \varepsilon)\right)$ (существование которого вытекает из принципа Шаудера) обладает требуемыми асимптотическими свойствами (2.21)-(2.24), в которых постоянные $T_{*}, y_{\text {int }}^{*}$, $y_{\max }^{*}$ и функция $x_{*}(t)$ заданы равенствами $(2.19),(2.20)$. И, наконец, проводя асимптотическое интегрирование системы в вариациях на рассматриваемом цикле, установим факт его экспоненциальной орбитальной устойчивости.

\section{§ 3. Построение асимптотики задачи Коши}

3.1. Основная геометрическая конструкция. Прежде чем приступить к асимптотическому анализу функций $x_{\left(x_{0}, \varphi\right)}(t, \varepsilon), y_{\left(x_{0}, \varphi\right)}(t, \varepsilon)$, сформулируем ряд дополнительных условий, которым должен удовлетворять отрезок $[a, b]$ из $(2.10),(2.25)$. Будем предполагать, что, во-первых, в дополнение к (2.15) имеют место соотношения

$$
\psi([a, b]) \subset(a, b), \quad \psi^{\prime}\left(x_{0}\right)<1 \quad \forall x_{0} \in[a, b]
$$

где $\psi$ - отображение (2.14). Во-вторых, запаздывание $h$ удовлетворяет аналогичному (2.17) неравенству

$$
h<h\left(x_{0}\right) \stackrel{\text { def }}{=}-\int_{x_{3}\left(x_{0}\right)}^{x_{1}\left(x_{0}\right)} \frac{d s}{f(s, 0)} \quad \forall x_{0} \in[a, b],
$$


где, напомним, $x_{1}\left(x_{0}\right), x_{3}\left(x_{0}\right)$ - функции, определяющиеся как неявные из уравнений (2.8) и (2.16) соответственно. В-третьих, выполняются аналогичные (2.18) требования

$$
g\left(x_{0}, y\right)>0, \quad g\left(x_{1}\left(x_{0}\right), y\right)<0 \quad \forall y \geqslant 0, \quad \forall x_{0} \in[a, b] .
$$

Подчеркнем, что в силу базовых условий 2.1-2.7 свойства (3.1)-(3.3) заведомо справедливы в точке $x_{0}=x_{0}^{*}$. Поэтому они сохраняются и на отрезке $x_{0} \in[a, b]$, если величины $a, b$ выбраны достаточно близкими к $x_{0}^{*}$.

Основная трудность при построении асимптотики пары функций $x_{\left(x_{0}, \varphi\right)}(t, \varepsilon)$, $y_{\left(x_{0}, \varphi\right)}(t, \varepsilon)$ связана с рассмотрением отрезка $0 \leqslant t \leqslant h$, на котором они меняются наиболее сложно. Для преодоления этой трудности ниже развивается некоторый специальный геометрический подход, сводящий проблему асимптотического интегрирования задачи Коши (1.25), (2.26) при $0 \leqslant t \leqslant h$ к анализу фазовой траектории некоторой вспомогательной автономной системы на плоскости.

Суть упомянутого геометрического подхода состоит в следующем. Обратимся к задаче Коши

$$
\dot{x}=f(x, y), \quad \varepsilon \dot{y}=\left[g(x, y)-\exp \frac{\varphi(t-h)}{\varepsilon}\right] y,\left.\quad x\right|_{t=0}=x_{0},\left.\quad y\right|_{t=0}=1
$$

для отыскания функций $x_{\left(x_{0}, \varphi\right)}(t, \varepsilon), y_{\left(x_{0}, \varphi\right)}(t, \varepsilon)$ при $0 \leqslant t \leqslant h$ и заметим, что в силу оценок, наложенных на $\varphi(t)$ (см. (2.25)), при любом фиксированном $\delta \in(0, h)$ справедливы неравенства

$$
\begin{gathered}
\max _{0 \leqslant t \leqslant h-\delta} \exp \frac{\varphi(t-h)}{\varepsilon} \leqslant \exp \left(-\frac{m_{1} \delta}{\varepsilon}\right), \\
\int_{h-\delta}^{h} \exp \frac{\varphi(t-h)}{\varepsilon} d t \leqslant \frac{\varepsilon}{m_{1}} .
\end{gathered}
$$

Отметим далее, что на эвристическом уровне строгости, опираясь на свойства $(3.5),(3.6)$, мы можем отбросить в (3.4) слагаемое, содержащее $\varphi(t)$, и перейти к рассмотрению более простой задачи

$$
\dot{x}=f(x, y), \quad \varepsilon \dot{y}=g(x, y) y,\left.\quad x\right|_{t=0}=x_{0},\left.\quad y\right|_{t=0}=1 .
$$

Как оказывается, эти эвристические соображения верны. А именно, ниже будет показано, что кривая

$$
\Gamma(\varepsilon)=\left\{(t, x, y): 0 \leqslant t \leqslant h, x=x_{\left(x_{0}, \varphi\right)}(t, \varepsilon), y=y_{\left(x_{0}, \varphi\right)}(t, \varepsilon)\right\}
$$

при $\varepsilon \rightarrow 0$ асимптотически близка к кривой

$$
\Lambda(\varepsilon)=\left\{(t, x, y): 0 \leqslant t \leqslant h, x=x\left(t, x_{0}, \varepsilon\right), y=y\left(t, x_{0}, \varepsilon\right)\right\},
$$

где $x\left(t, x_{0}, \varepsilon\right), y\left(t, x_{0}, \varepsilon\right)$ - компоненты решения задачи Коши (3.7).

Сравнительный асимптотический анализ кривых (3.8), (3.9) начнем с их участков $\Gamma_{1}(\varepsilon)$ и $\Lambda_{1}(\varepsilon)$, отвечающих значениям времени $0 \leqslant t \leqslant \tau_{\left(x_{0}, \varphi\right)}^{(1)}(\varepsilon)$ и 


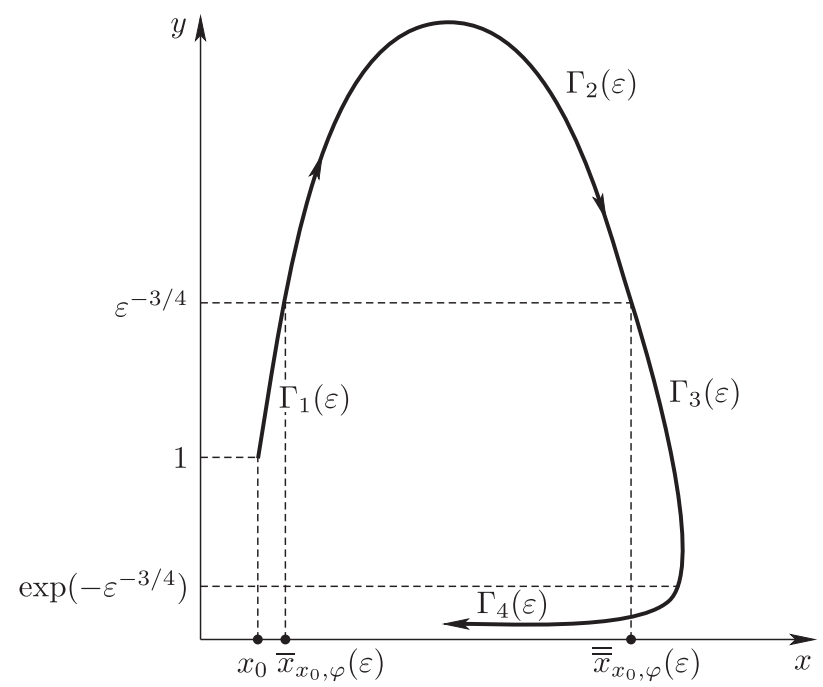

Рис. 5

$0 \leqslant t \leqslant \tau_{1}\left(x_{0}, \varepsilon\right)$, где $t=\tau_{\left(x_{0}, \varphi\right)}^{(1)}(\varepsilon), t=\tau_{1}\left(x_{0}, \varepsilon\right)$ - первые положительные корни уравнений

$$
y_{\left(x_{0}, \varphi\right)}(t, \varepsilon)=\varepsilon^{-3 / 4}, \quad y\left(t, x_{0}, \varepsilon\right)=\varepsilon^{-3 / 4} .
$$

Поскольку, как будет показано ниже, моменты времени $\tau_{\left(x_{0}, \varphi\right)}^{(1)}(\varepsilon), \tau_{1}\left(x_{0}, \varepsilon\right)$ асимптотически малы и на рассматриваемых промежутках изменения $t$ компоненты $y_{\left(x_{0}, \varphi\right)}(t, \varepsilon), y\left(t, x_{0}, \varepsilon\right)$ монотонно растут от 1 до $\varepsilon^{-3 / 4}$, то участки $\Gamma_{1}(\varepsilon)$, $\Lambda_{1}(\varepsilon)$ будем называть участками взлета. Примерный вид $\Gamma_{1}(\varepsilon)$ показан на рис. 5 , где представлена проекция кривой $\Gamma(\varepsilon)$ на плоскость $(x, y)$.

В формулируемом ниже утверждении в качестве параметра на $\Lambda_{1}(\varepsilon)$ вместо времени $t$ взята переменная $y \in\left[1, \varepsilon^{-3 / 4}\right]$.

ЛЕмма 3.1. Участок взлета $\Lambda_{1}(\varepsilon)$ задается равенством

$$
\Lambda_{1}(\varepsilon)=\left\{(t, x, y): t=\theta_{1}\left(y, x_{0}, \varepsilon\right), x=\widetilde{x}\left(y, x_{0}, \varepsilon\right), 1 \leqslant y \leqslant \varepsilon^{-3 / 4}\right\},
$$

где функиии $\theta_{1}\left(y, x_{0}, \varepsilon\right), \widetilde{x}\left(y, x_{0}, \varepsilon\right)$ при $\varepsilon \rightarrow 0$ допускают равномерные по $x_{0} \in$ $[a, b], y \in\left[1, \varepsilon^{-3 / 4}\right]$ асимптотические представления

$$
\begin{gathered}
\theta_{1}\left(y, x_{0}, \varepsilon\right)=\varepsilon \int_{1}^{y} \frac{d s}{s g\left(x_{0}, s\right)}+O\left(\varepsilon^{5 / 4} \ln \frac{1}{\varepsilon}\right), \\
\frac{\partial \theta_{1}}{\partial x_{0}}\left(y, x_{0}, \varepsilon\right)=\varepsilon\left(\frac{\partial}{\partial x_{0}} \int_{1}^{y} \frac{d s}{s g\left(x_{0}, s\right)}\right)+O\left(\varepsilon^{5 / 4} \ln \frac{1}{\varepsilon}\right), \\
\frac{\partial \theta_{1}}{\partial y}\left(y, x_{0}, \varepsilon\right)=\frac{\varepsilon}{y}\left(\frac{1}{g\left(x_{0}, y\right)}+O\left(\varepsilon^{1 / 4}\right)\right), \\
\frac{\partial^{2} \theta_{1}}{\partial x_{0} \partial y}\left(y, x_{0}, \varepsilon\right)=\frac{\varepsilon}{y}\left(\frac{\partial}{\partial x_{0}}\left(\frac{1}{g\left(x_{0}, y\right)}\right)+O\left(\varepsilon^{1 / 4}\right)\right),
\end{gathered}
$$




$$
\begin{gathered}
\widetilde{x}\left(y, x_{0}, \varepsilon\right)=x_{0}+O\left(\varepsilon^{1 / 4}\right), \quad \frac{\partial}{\partial x_{0}} \widetilde{x}\left(y, x_{0}, \varepsilon\right)=1+O\left(\varepsilon^{1 / 4}\right), \\
\frac{\partial}{\partial y} \widetilde{x}\left(y, x_{0}, \varepsilon\right)=O(\varepsilon) .
\end{gathered}
$$

ДокАЗАТЕЛЬство. Из (3.7) очевидным образом следует, что на кривой $\Lambda_{1}(\varepsilon)$ переменная $x$ как функция от $y$ должна удовлетворять задаче Коши

$$
\frac{d x}{d y}=\varepsilon F(x, y),\left.\quad x\right|_{y=1}=x_{0}
$$

с правой частью

$$
F(x, y)=\frac{f(x, y)}{y g(x, y)} .
$$

Для выявления асимптотических свойств решения этой задачи проделаем некоторые предварительные построения.

Фиксируем постоянные $a^{\prime}<a, b^{\prime}>b$, достаточно близкие к $a, b$ и такие, что на отрезке $a^{\prime} \leqslant x \leqslant b^{\prime}$ сохраняется первое неравенство (3.3), т.е.

$$
g(x, y)>0 \quad \forall y \geqslant 0, \quad \forall x \in\left[a^{\prime}, b^{\prime}\right] .
$$

Рассмотрим, далее, множество

$$
\Sigma=\left\{(x, y): a^{\prime} \leqslant x \leqslant b^{\prime}, 1 \leqslant y \leqslant \varepsilon^{-3 / 4}\right\} .
$$

Из явного вида (3.18) функции $F$ и свойств $(2.1)-(2.3),(3.19)$ вытекает, что

$$
|F(x, y)| \leqslant M_{1}, \quad\left|F_{x}^{\prime}(x, y)\right| \leqslant M_{2}, \quad\left|F_{x x}^{\prime \prime}(x, y)\right| \leqslant M_{3} \quad \forall(x, y) \in \Sigma .
$$

Здесь и ниже через $M, M_{1}, M_{2}$ и т.д. обозначаются различные универсальные положительные постоянные, точные значения которых несущественны. В частности, фигурирующие в (3.21) константы $M_{1}, M_{2}, M_{3}$ не зависят от $x, y, \varepsilon$, но зависят от $a^{\prime}, b^{\prime}$ из (3.19).

Кроме множества (3.20) нам потребуется еще банахово пространство $X$. Его элементами являются функции $x\left(y, x_{0}\right)$, непрерывные по совокупности переменных $\left(y, x_{0}\right) \in\left[1, \varepsilon^{-3 / 4}\right] \times[a, b]$ вместе с частной производной $\partial x / \partial x_{0}$. Норму в $X$ зададим равенством

$$
\|x\|=\max _{\left(y, x_{0}\right) \in\left[1, \varepsilon^{-3 / 4}\right] \times[a, b]}\left(\left|x\left(y, x_{0}\right)\right|+\left|\frac{\partial x}{\partial x_{0}}\left(y, x_{0}\right)\right|\right) .
$$

Обратимся теперь непосредственно к задаче Коши (3.17) и будем искать ее решение $x=\widetilde{x}\left(y, x_{0}, \varepsilon\right)$ как неподвижную точку оператора

$$
\mathscr{L}_{0}(x)\left(y, x_{0}\right)=x_{0}+\varepsilon \int_{1}^{y} F\left(x\left(s, x_{0}\right), s\right) d s,
$$

определенного на множестве функций

$$
\left\{x\left(y, x_{0}\right): a^{\prime} \leqslant x\left(y, x_{0}\right) \leqslant b^{\prime},\left|\frac{\partial x}{\partial x_{0}}\left(y, x_{0}\right)\right| \leqslant 2\right\} \subset X
$$


и принимающего значения в $X$. Несложная проверка показывает, что для любых элементов $x\left(y, x_{0}\right), x_{1}\left(y, x_{0}\right), x_{2}\left(y, x_{0}\right)$ из области определения (3.23) оператора (3.22) справедливы оценки

$$
\begin{gathered}
\left|\mathscr{L}_{0}(x)\left(y, x_{0}\right)-x_{0}\right| \leqslant \varepsilon M_{1}(y-1), \quad\left|\frac{\partial}{\partial x_{0}} \mathscr{L}_{0}(x)\left(y, x_{0}\right)-1\right| \leqslant 2 \varepsilon M_{2}(y-1), \\
\left|\mathscr{L}_{0}\left(x_{1}\right)\left(y, x_{0}\right)-\mathscr{L}_{0}\left(x_{2}\right)\left(y, x_{0}\right)\right| \leqslant \varepsilon M_{2} \int_{1}^{y}\left|x_{1}\left(s, x_{0}\right)-x_{2}\left(s, x_{0}\right)\right| d s \\
\left|\frac{\partial}{\partial x_{0}} \mathscr{L}_{0}\left(x_{1}\right)\left(y, x_{0}\right)-\frac{\partial}{\partial x_{0}} \mathscr{L}_{0}\left(x_{2}\right)\left(y, x_{0}\right)\right| \leqslant \varepsilon M_{2} \int_{1}^{y}\left|\frac{\partial x_{1}}{\partial x_{0}}\left(s, x_{0}\right)-\frac{\partial x_{2}}{\partial x_{0}}\left(s, x_{0}\right)\right| d s \\
+2 \varepsilon M_{3} \int_{1}^{y}\left|x_{1}\left(s, x_{0}\right)-x_{2}\left(s, x_{0}\right)\right| d s \quad \forall y \in\left[1, \varepsilon^{-3 / 4}\right], \quad \forall x_{0} \in[a, b],
\end{gathered}
$$

где $M_{1}, M_{2}, M_{3}$ - постоянные из (3.21). А отсюда очевидным образом вытекают следующие факты, имеющие место при всех достаточно малых $\varepsilon>0$.

Во-первых, оператор $\mathscr{L}_{0}$ преобразует множество (3.23) в себя и является сжимающим (с константой сжатия порядка $\left.\varepsilon^{1 / 4}\right)$. Во-вторых, для его неподвижной точки $\widetilde{x}\left(y, x_{0}, \varepsilon\right)$ (существование и единственность которой гарантирует принцип сжимающих отображений) равномерно по $y \in\left[1, \varepsilon^{-3 / 4}\right], x_{0} \in[a, b]$ справедливы требуемые асимптотические представления (3.16). Что же касается функции $\theta_{1}\left(y, x_{0}, \varepsilon\right)$, то она задается явным выражением

$$
\theta_{1}\left(y, x_{0}, \varepsilon\right)=\varepsilon \int_{1}^{y} \frac{d s}{s g\left(\widetilde{x}\left(s, x_{0}, \varepsilon\right), s\right)},
$$

из которого с учетом равенств (3.16) и свойств $(2.1),(2.3)$ функции $g(x, y)$ асимптотические формулы (3.12)-(3.15) выводятся автоматически. Лемма 3.1 доказана.

Перейдем теперь от кривой $\Lambda_{1}(\varepsilon)$ к участку $\Gamma_{1}(\varepsilon)$ кривой (3.8). Как оказывается, он экспоненциально близок к $\Lambda_{1}(\varepsilon)$. Точнее говоря, справедливо следующее утверждение.

ЛЕмма 3.2. Участок $\Gamma_{1}(\varepsilon)$ задается аналогичным (3.11) равенством

$$
\Gamma_{1}(\varepsilon)=\left\{(t, x, y): t=\theta_{x_{0}, \varphi}^{(1)}(y, \varepsilon), x=\widetilde{x}_{x_{0}, \varphi}(y, \varepsilon), 1 \leqslant y \leqslant \varepsilon^{-3 / 4}\right\}
$$

где функиии $\theta_{x_{0}, \varphi}^{(1)}(y, \varepsilon), \widetilde{x}_{x_{0}, \varphi}(y, \varepsilon)$ таковы, что при $\varepsilon \rightarrow 0$ равномерно по начальным условиям $\left(x_{0}, \varphi\right) \in S$ и по $y \in\left[1, \varepsilon^{-3 / 4}\right]$

$$
\begin{gathered}
\left(\theta_{x_{0}, \varphi}^{(1)}(y, \varepsilon), \frac{\partial}{\partial y} \theta_{x_{0}, \varphi}^{(1)}(y, \varepsilon)\right)=\left(\theta_{1}\left(y, x_{0}, \varepsilon\right), \frac{\partial}{\partial y} \theta_{1}\left(y, x_{0}, \varepsilon\right)\right)+O\left(\exp \left(-\frac{M}{\varepsilon}\right)\right), \\
\left(\widetilde{x}_{x_{0}, \varphi}(y, \varepsilon), \frac{\partial}{\partial y} \widetilde{x}_{x_{0}, \varphi}(y, \varepsilon)\right)=\left(\widetilde{x}\left(y, x_{0}, \varepsilon\right), \frac{\partial}{\partial y} \widetilde{x}\left(y, x_{0}, \varepsilon\right)\right)+O\left(\exp \left(-\frac{M}{\varepsilon}\right)\right), \\
M=\mathrm{const}>0 .
\end{gathered}
$$


ДокАЗАтЕльство. Предположим, что на кривой $\Gamma_{1}(\varepsilon)$ переменная $t$ связана с $у$ соотношением

$$
t=\theta(y)
$$

где $\theta(y)$ - пока произвольная (подлежащая определению) функция из множества

$$
\Omega=\left\{\theta(y) \in C\left[1, \varepsilon^{-3 / 4}\right]: 0 \leqslant \theta(y) \leqslant M \varepsilon \ln \frac{1}{\varepsilon}, \theta(1)=0\right\},
$$

а $M>0$ - некоторая универсальная константа. Тогда для определения $x$ как функции от $y$ приходим к аналогичной (3.17) задаче Коши

$$
\frac{d x}{d y}=\varepsilon F(x, y, \theta(y), \varepsilon),\left.\quad x\right|_{y=1}=x_{0},
$$

где

$$
F(x, y, \theta, \varepsilon)=\frac{f(x, y)}{y[g(x, y)-\exp (\varphi(\theta-h) / \varepsilon)]} .
$$

Как и выше, остановимся сначала на некоторых необходимых для анализа задачи (3.29) свойствах функции (3.30). Из ее явного вида и соотношений (2.1)-(2.3), (3.5), (3.19) вытекает, что $\forall(x, y) \in \Sigma, \forall \theta \in \Omega$, где $\Sigma, \Omega$ - множества (3.20), (3.28), и при всех достаточно малых $\varepsilon>0$ выполняются оценки вида

$$
\begin{gathered}
|F(x, y, \theta(y), \varepsilon)| \leqslant M_{1}, \quad\left|F_{x}^{\prime}(x, y, \theta(y), \varepsilon)\right| \leqslant M_{2}, \\
|F(x, y, \theta(y), \varepsilon)-F(x, y)| \leqslant M_{3} \exp \left(-\frac{M_{4}}{\varepsilon}\right) .
\end{gathered}
$$

Здесь $M_{j}=$ const $>0, j=1, \ldots, 4$, а $F(x, y)$ - функция (3.18).

Перейдем затем непосредственно к рассмотрению задачи Коши (3.29). В данном случае ее решение $x=x_{x_{0}, \varphi, \theta}(y, \varepsilon)$ будем искать как неподвижную точку аналогичного (3.22) оператора

$$
\mathscr{L}_{0}(x)(y)=x_{0}+\varepsilon \int_{1}^{y} F(x(s), s, \theta(s), \varepsilon) d s,
$$

действующего в пространстве $C\left[1, \varepsilon^{-3 / 4}\right]$ функций $x(y)$ одной переменной $y$ ( $x_{0}$ здесь рассматривается как параметр). Из неравенств (3.31) очевидным образом следует, что, во-первых, оператор (3.32) преобразует в себя замкнутое множество

$$
\left\{x(y): a^{\prime} \leqslant x(y) \leqslant b^{\prime}\right\} \subset C\left[1, \varepsilon^{-3 / 4}\right]
$$

и является сжимающим; во-вторых, для его неподвижной точки $x_{x_{0}, \varphi, \theta}(y, \varepsilon)$ справедливы оценки

$$
\begin{aligned}
& \left|\frac{\partial}{\partial y} x_{x_{0}, \varphi, \theta}(y, \varepsilon)-\frac{\partial}{\partial y} \widetilde{x}\left(y, x_{0}, \varepsilon\right)\right| \\
& \quad \leqslant \varepsilon M_{2}\left|x_{x_{0}, \varphi, \theta}(y, \varepsilon)-\widetilde{x}\left(y, x_{0}, \varepsilon\right)\right|+\varepsilon M_{3} \exp \left(-\frac{M_{4}}{\varepsilon}\right), \\
& \quad\left|x_{x_{0}, \varphi, \theta}(y, \varepsilon)-\widetilde{x}\left(y, x_{0}, \varepsilon\right)\right| \\
& \quad \leqslant \varepsilon M_{2} \int_{1}^{y}\left|x_{x_{0}, \varphi, \theta}(s, \varepsilon)-\widetilde{x}\left(s, x_{0}, \varepsilon\right)\right| d s+\varepsilon(y-1) M_{3} \exp \left(-\frac{M_{4}}{\varepsilon}\right),
\end{aligned}
$$


где $\widetilde{x}\left(y, x_{0}, \varepsilon\right)$ - функция из (3.11), $M_{2}, M_{3}$ - константы из (3.31). Опираясь, далее, на лемму Гронуолла-Беллмана, окончательно получаем

$$
\begin{gathered}
\left|x_{x_{0}, \varphi, \theta}(y, \varepsilon)-\widetilde{x}\left(y, x_{0}, \varepsilon\right)\right|+\left|\frac{\partial}{\partial y} x_{x_{0}, \varphi, \theta}(y, \varepsilon)-\frac{\partial}{\partial y} \widetilde{x}\left(y, x_{0}, \varepsilon\right)\right| \leqslant \exp \left(-\frac{M}{\varepsilon}\right), \\
M=\text { const }>0, \quad y \in\left[1, \varepsilon^{-3 / 4}\right] .
\end{gathered}
$$

Разберемся теперь с вопросом об отыскании фигурирующей в (3.27) функции $\theta(y)$. Из уравнения для компоненты $y$ (см. (3.4)) следует, что она должна удовлетворять интегральному уравнению

$$
\theta(y)=\mathscr{L}_{1}(\theta)(y) \stackrel{\text { def }}{=} \varepsilon \int_{1}^{y} \frac{1}{s} G\left(x_{x_{0}, \varphi, \theta}(s, \varepsilon), s, \theta(s), \varepsilon\right) d s,
$$

где функция $G(x, y, \theta, \varepsilon)$ задается аналогичной (3.30) формулой

$$
G(x, y, \theta, \varepsilon)=\frac{1}{g(x, y)-\exp (\varphi(\theta-h) / \varepsilon)}
$$

и удовлетворяет аналогичным (3.31) неравенствам

$$
\begin{gathered}
|G(x, y, \theta(y), \varepsilon)| \leqslant M_{1}, \quad\left|G_{x}^{\prime}(x, y, \theta(y), \varepsilon)\right| \leqslant M_{2} \\
\left|G(x, y, \theta(y), \varepsilon)-\frac{1}{g(x, y)}\right| \leqslant M_{3} \exp \left(-\frac{M_{4}}{\varepsilon}\right) \quad \forall(x, y) \in \Sigma, \quad \forall \theta(y) \in \Omega .
\end{gathered}
$$

Опираясь на свойства (3.39) функции (3.38), нетрудно убедиться, что

$$
\begin{aligned}
& \left|G\left(x_{x_{0}, \varphi, \theta}(y, \varepsilon), y, \theta(y), \varepsilon\right)-\frac{1}{g\left(\widetilde{x}\left(y, x_{0}, \varepsilon\right), y\right)}\right| \\
& \quad \leqslant M_{2}\left|x_{x_{0}, \varphi, \theta}(y, \varepsilon)-\widetilde{x}\left(y, x_{0}, \varepsilon\right)\right|+M_{3} \exp \left(-\frac{M_{4}}{\varepsilon}\right) \quad \forall y \in\left[1, \varepsilon^{-3 / 4}\right],
\end{aligned}
$$

где постоянные $M_{2}, M_{3}$ те же, что и в (3.39). Объединяя, далее, оценки (3.40), (3.36), приходим к выводу, что равномерно по $y \in\left[1, \varepsilon^{-3 / 4}\right], \theta(y) \in \Omega,\left(x_{0}, \varphi\right) \in S$

$$
\begin{gathered}
\mathscr{L}_{1}(\theta)(y)=\theta_{1}\left(y, x_{0}, \varepsilon\right)+O\left(\exp \left(-\frac{M}{\varepsilon}\right)\right), \\
\frac{\partial}{\partial y} \mathscr{L}_{1}(\theta)(y)=\frac{\partial \theta_{1}}{\partial y}\left(y, x_{0}, \varepsilon\right)+O\left(\exp \left(-\frac{M}{\varepsilon}\right)\right), \quad \varepsilon \rightarrow 0, \quad M=\text { const }>0,
\end{gathered}
$$

где, напомним, $\theta_{1}\left(y, x_{0}, \varepsilon\right)$ - функция (3.24).

Для завершения обоснования леммы 3.2 заметим, что в силу (3.41) и уже установленных асимптотических представлений (3.12)-(3.15) оператор $\mathscr{L}_{1}$ из (3.37), очевидным образом являющийся компактным, при подходящем выборе постоянной $M$ в (3.28), а именно, при

$$
M>\frac{3}{4 b_{0}\left(x_{0}\right)},
$$


преобразует в себя замкнутое ограниченное выпуклое множество функций $\Omega \subset$ $C\left[1, \varepsilon^{-3 / 4}\right]$. Тем самым, согласно принципу Шаудера он допускает в $\Omega$ хотя бы одну неподвижную точку $\theta_{x_{0}, \varphi}^{(1)}(y, \varepsilon)$, для которой заведомо справедлива нужная асимптотическая формула из (3.26). Что же касается фигурирующей в (3.25) функции $\widetilde{x}_{x_{0}, \varphi}(y, \varepsilon)$, то она задается равенством

$$
\widetilde{x}_{x_{0}, \varphi}(y, \varepsilon)=\left.x_{x_{0}, \varphi, \theta}(y, \varepsilon)\right|_{\theta=\theta_{x_{0}, \varphi}^{(1)}}
$$

и в силу (3.36) также обладает требуемым свойством из (3.26). Лемма 3.2 доказана.

Уместно отметить, что на самом деле оператор $\mathscr{L}_{1}$ имеет в $\Omega$ единственную неподвижную точку, поскольку единственна функция $y_{\left(x_{0}, \varphi\right)}(t, \varepsilon)$, определяющаяся как неявная из уравнения $t=\theta_{x_{0}, \varphi}^{(1)}(y, \varepsilon)$.

Завершая рассмотрение участков взлета $\Gamma_{1}(\varepsilon), \Lambda_{1}(\varepsilon)$, напомним, что им соответствуют значения $t \in\left[0, \tau_{\left(x_{0}, \varphi\right)}^{(1)}(\varepsilon)\right]$ и $t \in\left[0, \tau_{1}\left(x_{0}, \varepsilon\right)\right]$, где $\tau_{\left(x_{0}, \varphi\right)}^{(1)}(\varepsilon), \tau_{1}\left(x_{0}, \varepsilon\right)-$ первые положительные корни уравнений (3.10). Из формул (3.12), (3.13), (3.26) следует, что для этих корней справедливы равномерные по $\left(x_{0}, \varphi\right) \in S$ асимптотические представления

$$
\begin{gathered}
\left.\tau_{1}\left(x_{0}, \varepsilon\right) \stackrel{\text { def }}{=} \theta_{1}\left(y, x_{0}, \varepsilon\right)\right|_{y=\varepsilon^{-3 / 4}}=\frac{3}{4 b_{0}\left(x_{0}\right)} \varepsilon \ln \frac{1}{\varepsilon}+O(\varepsilon), \\
\frac{\partial}{\partial x_{0}} \tau_{1}\left(x_{0}, \varepsilon\right)=\frac{\partial}{\partial x_{0}}\left(\frac{3}{4 b_{0}\left(x_{0}\right)} \varepsilon \ln \frac{1}{\varepsilon}\right)+O(\varepsilon), \\
\left.\tau_{\left(x_{0}, \varphi\right)}^{(1)}(\varepsilon) \stackrel{\text { def }}{=} \theta_{x_{0}, \varphi}^{(1)}(y, \varepsilon)\right|_{y=\varepsilon^{-3 / 4}}=\tau_{1}\left(x_{0}, \varepsilon\right)+O\left(\exp \left(-\frac{M}{\varepsilon}\right)\right), \\
M=\text { const }>0 .
\end{gathered}
$$

Обратимся далее к очередным участкам $\Gamma_{2}(\varepsilon), \Lambda_{2}(\varepsilon)$ кривых (3.8), (3.9), отвечающим значениям $t \in\left[\tau_{\left(x_{0}, \varphi\right)}^{(1)}(\varepsilon), \tau_{\left(x_{0}, \varphi\right)}^{(2)}(\varepsilon)\right]$ и $t \in\left[\tau_{1}\left(x_{0}, \varepsilon\right), \tau_{2}\left(x_{0}, \varepsilon\right)\right]$, где $\tau_{\left(x_{0}, \varphi\right)}^{(2)}(\varepsilon), \tau_{2}\left(x_{0}, \varepsilon\right)$ - вторые положительные корни уравнений (3.10). Данные участки характерны тем, что они целиком лежат в полупространстве $\{(t, x, y)$ : $\left.y \geqslant \varepsilon^{-3 / 4}\right\}$ и при движении по ним, которое длится время порядка $\varepsilon \ln (1 / \varepsilon)$, точка $(t, x, y)$ сначала покидает плоскость $y=\varepsilon^{-3 / 4}$, а затем снова возвращается на нее (см. рис. 5). По аналогии со случаем системы (1.1) эти участки будем называть участками поворота, а в качестве параметра на них возьмем переменную $x$.

Лемма 3.3. Для участка поворота $\Lambda_{2}(\varepsilon)$ имеет место равенство

$$
\Lambda_{2}(\varepsilon)=\left\{(t, x, y): t=\theta_{2}\left(x, x_{0}, \varepsilon\right), y=\frac{u\left(x, x_{0}, \varepsilon\right)}{\varepsilon}, \bar{x}\left(x_{0}, \varepsilon\right) \leqslant x \leqslant \overline{\bar{x}}\left(x_{0}, \varepsilon\right)\right\}
$$

где

$$
\begin{gathered}
\left.u\left(x, x_{0}, \varepsilon\right)\right|_{x=\bar{x}\left(x_{0}, \varepsilon\right), \overline{\bar{x}}\left(x_{0}, \varepsilon\right)}=\varepsilon^{1 / 4}, \quad u\left(x, x_{0}, \varepsilon\right)>\varepsilon^{1 / 4} \\
n p u \quad x \in\left(\bar{x}\left(x_{0}, \varepsilon\right), \overline{\bar{x}}\left(x_{0}, \varepsilon\right)\right),\left.\quad \theta_{2}\left(x, x_{0}, \varepsilon\right)\right|_{x=\bar{x}\left(x_{0}, \varepsilon\right)}=\tau_{1}\left(x_{0}, \varepsilon\right) .
\end{gathered}
$$


Кроме того, при $\varepsilon \rightarrow 0$ для функиий $\bar{x}\left(x_{0}, \varepsilon\right), \overline{\bar{x}}\left(x_{0}, \varepsilon\right)$ справедливы равномерные по $x_{0} \in[a, b]$ асимптотические представления

$$
\begin{gathered}
\bar{x}\left(x_{0}, \varepsilon\right)=\left.\left(x+\frac{a_{0}(x)}{b_{0}(x)} \varepsilon^{1 / 4}\right)\right|_{x=x_{0}}+O(\sqrt{\varepsilon}) \\
\frac{\partial}{\partial x_{0}} \bar{x}\left(x_{0}, \varepsilon\right)=\frac{\partial}{\partial x_{0}}\left[\left.\left(x+\frac{a_{0}(x)}{b_{0}(x)} \varepsilon^{1 / 4}\right)\right|_{x=x_{0}}\right]+O(\sqrt{\varepsilon}), \\
\overline{\bar{x}}\left(x_{0}, \varepsilon\right)=\left.\left(x+\frac{a_{0}(x)}{b_{0}(x)} \varepsilon^{1 / 4}\right)\right|_{x=x_{1}\left(x_{0}\right)}+O(\sqrt{\varepsilon}), \\
\frac{\partial}{\partial x_{0}} \overline{\bar{x}}\left(x_{0}, \varepsilon\right)=\frac{\partial}{\partial x_{0}}\left[\left.\left(x+\frac{a_{0}(x)}{b_{0}(x)} \varepsilon^{1 / 4}\right)\right|_{x=x_{1}\left(x_{0}\right)}\right]+O(\sqrt{\varepsilon}),
\end{gathered}
$$

а для функиий $u\left(x, x_{0}, \varepsilon\right), \theta_{2}\left(x, x_{0}, \varepsilon\right)$ - равномерные по переменным $x_{0} \in[a, b]$, $x \in\left[\bar{x}\left(x_{0}, \varepsilon\right), \overline{\bar{x}}\left(x_{0}, \varepsilon\right)\right]$ представления

$$
\begin{gathered}
u\left(x, x_{0}, \varepsilon\right)=\gamma_{0}\left(x, x_{0}\right)+O(\sqrt{\varepsilon}), \quad \frac{\partial}{\partial x} u\left(x, x_{0}, \varepsilon\right)=\frac{b_{0}(x)}{a_{0}(x)}+O(\sqrt{\varepsilon}), \\
\frac{\partial}{\partial x_{0}} u\left(x, x_{0}, \varepsilon\right)=-\frac{b_{0}\left(x_{0}\right)}{a_{0}\left(x_{0}\right)}+O(\sqrt{\varepsilon}) \\
\theta_{2}\left(x, x_{0}, \varepsilon\right)=\tau_{1}\left(x_{0}, \varepsilon\right)+\varepsilon \int_{\bar{x}\left(x_{0}, \varepsilon\right)}^{x} \frac{d s}{a_{0}(s) \gamma_{0}\left(s, x_{0}\right)}+O\left(\varepsilon^{5 / 4}\right) \\
\frac{\partial}{\partial x_{0}} \theta_{2}\left(x, x_{0}, \varepsilon\right)=\frac{\partial}{\partial x_{0}}\left(\tau_{1}\left(x_{0}, \varepsilon\right)+\varepsilon \int_{\bar{x}\left(x_{0}, \varepsilon\right)}^{x} \frac{d s}{a_{0}(s) \gamma_{0}\left(s, x_{0}\right)}\right)+O(\varepsilon) \\
\frac{\partial}{\partial x} \theta_{2}\left(x, x_{0}, \varepsilon\right)=\frac{\varepsilon}{a_{0}(x) \gamma_{0}\left(x, x_{0}\right)}+O\left(\frac{\varepsilon^{3 / 2}}{\gamma_{0}^{2}\left(x, x_{0}\right)}\right)>0 \\
\forall x \in\left[\bar{x}\left(x_{0}, \varepsilon\right), \overline{\bar{x}}\left(x_{0}, \varepsilon\right)\right] \\
\frac{\partial}{\partial x \partial x_{0}} \theta_{2}\left(x, x_{0}, \varepsilon\right)=\varepsilon \frac{\partial}{\partial x_{0}}\left(\frac{1}{a_{0}(x) \gamma_{0}\left(x, x_{0}\right)}\right)+O\left(\frac{\varepsilon^{3 / 2}}{\gamma_{0}^{3}\left(x, x_{0}\right)}\right)
\end{gathered}
$$

где $\gamma_{0}\left(x, x_{0}\right)$ - функиия из $(2.7)$.

ДокАзАтельство. Отметим прежде всего, что фигурирующее в (3.45) значение $x=\bar{x}\left(x_{0}, \varepsilon\right)$ определяется посредством равенства

$$
\bar{x}\left(x_{0}, \varepsilon\right)=\left.\widetilde{x}\left(y, x_{0}, \varepsilon\right)\right|_{y=\varepsilon^{-3 / 4}},
$$

где $\widetilde{x}\left(y, x_{0}, \varepsilon\right)$ - функция из (3.11). Однако для обоснования асимптотических формул (3.47), (3.48) полученной ранее информации (3.16) о данной функции очевидным образом недостаточно. В связи с этим обратимся к интегральному уравнению

$$
\widetilde{x}\left(y, x_{0}, \varepsilon\right)=x_{0}+\varepsilon \int_{1}^{y} F\left(\widetilde{x}\left(s, x_{0}, \varepsilon\right), s\right) d s,
$$

где $F(x, y)$ - функция (3.18), и учтем в его правой части соотношения (3.16). В результате после несложных преобразований получаем уточненные версии 
асимптотических формул для $\widetilde{x}\left(y, x_{0}, \varepsilon\right)$, имеющие вид

$$
\begin{gathered}
\widetilde{x}\left(y, x_{0}, \varepsilon\right)=x_{0}+\varepsilon \int_{1}^{y} \frac{f\left(x_{0}, s\right)}{s g\left(x_{0}, s\right)} d s+O\left(\varepsilon^{5 / 4} y\right), \\
\frac{\partial}{\partial x_{0}} \widetilde{x}\left(y, x_{0}, \varepsilon\right)=1+\varepsilon \int_{1}^{y} \frac{\partial}{\partial x_{0}}\left(\frac{f\left(x_{0}, s\right)}{s g\left(x_{0}, s\right)}\right) d s+O\left(\varepsilon^{5 / 4} y\right), \\
1 \leqslant y \leqslant \varepsilon^{-3 / 4} .
\end{gathered}
$$

Полагая затем в (3.57) $y=\varepsilon^{-3 / 4}$ и опираясь на свойства $(2.1)-(2.3)$, для функции (3.56) приходим к нужным асимптотическим представлениям $(3.47),(3.48)$. Добавим еще, что так как $a_{0}\left(x_{0}\right) / b_{0}\left(x_{0}\right)>0$, то автоматически $\bar{x}\left(x_{0}, \varepsilon\right)>x_{0}$.

Для отыскания оставшихся функций $u\left(x, x_{0}, \varepsilon\right), \overline{\bar{x}}\left(x_{0}, \varepsilon\right), \theta_{2}\left(x, x_{0}, \varepsilon\right)$ обратимся к системе из (3.7), выполним в ней замену $y=u / \varepsilon$ и переразложим ее правые части по $\varepsilon$ с учетом свойств (2.1). В результате убедимся, что $u=u\left(x, x_{0}, \varepsilon\right)$ удовлетворяет задаче Коши вида

$$
\frac{d u}{d x}=\frac{b_{0}(x)}{a_{0}(x)}+\Phi(u, x, \varepsilon),\left.\quad u\right|_{x=\bar{x}\left(x_{0}, \varepsilon\right)}=\varepsilon^{1 / 4} .
$$

При этом явное выражение для функции $\Phi(u, x, \varepsilon)$ опустим, так как в дальнейшем оно не потребуется. Отметим только, что $\forall(x, u) \in \Sigma$, где

$$
\begin{array}{r}
\Sigma=\left\{(x, u): x \in J, u \geqslant d \varepsilon^{1 / 4}\right\}, \quad d=\text { const } \in(0,1), \\
J=\left[x_{0}+c_{1} \varepsilon^{1 / 4}, x_{1}\left(x_{0}\right)-c_{2} \varepsilon^{1 / 4}\right], \quad c_{1}, c_{2}=\text { const }>0, \\
0<c_{1}<\min _{a \leqslant x_{0} \leqslant b} \frac{a_{0}\left(x_{0}\right)}{b_{0}\left(x_{0}\right)}, \quad 0<c_{2}<\min _{a \leqslant x_{0} \leqslant b}\left(-\left.\frac{a_{0}(x)}{b_{0}(x)}\right|_{x=x_{1}\left(x_{0}\right)}\right),
\end{array}
$$

имеют место оценки вида

$$
|\Phi(u, x, \varepsilon)| \leqslant M_{1} \frac{\varepsilon}{u}, \quad\left|\Phi_{u}^{\prime}(u, x, \varepsilon)\right| \leqslant M_{2} \frac{\varepsilon}{u^{2}} .
$$

Свойства (3.62) свидетельствуют о том, что задача (3.58) представляет собой регулярное возмущение системы

$$
\frac{d u}{d x}=\frac{b_{0}(x)}{a_{0}(x)},\left.\quad u\right|_{x=\bar{x}\left(x_{0}, \varepsilon\right)}=\varepsilon^{1 / 4} .
$$

Тем самым, решение $u\left(x, x_{0}, \varepsilon\right)$ этой задачи при тех значениях $x \in J$, для которых

$$
\left(x, u\left(x, x_{0}, \varepsilon\right)\right) \in \Sigma,
$$

допускает асимптотические представления

$$
\begin{gathered}
u\left(x, x_{0}, \varepsilon\right)=\varepsilon^{1 / 4}+\int_{\bar{x}\left(x_{0}, \varepsilon\right)}^{x} \frac{b_{0}(s)}{a_{0}(s)} d s+O\left(\varepsilon^{3 / 4}\right), \\
\frac{\partial}{\partial x} u\left(x, x_{0}, \varepsilon\right)=\frac{b_{0}(x)}{a_{0}(x)}+O\left(\varepsilon^{3 / 4}\right), \\
\frac{\partial}{\partial x_{0}} u\left(x, x_{0}, \varepsilon\right)=-\left(\left.\frac{b_{0}(x)}{a_{0}(x)}\right|_{x=\bar{x}\left(x_{0}, \varepsilon\right)}\right) \frac{\partial \bar{x}}{\partial x_{0}}\left(x_{0}, \varepsilon\right)+O(\sqrt{\varepsilon}) .
\end{gathered}
$$


Следует отметить, что соотношения (3.64)-(3.66) преобразуются к требуемому виду (3.51). Для того чтобы убедиться в этом, достаточно подставить в (3.64), (3.66) уже установленные асимптотические формулы $(3.47),(3.48)$ и переразложить результат по $\varepsilon$.

Зададимся теперь вопросом о том, при каких же именно значениях $x$ выполняется априорное условие (3.63). В связи с этим обратим внимание, что в силу (2.9)

$$
\begin{gathered}
\gamma_{0}\left(x, x_{0}\right)>0 \quad \text { при } x_{0}<x<x_{1}\left(x_{0}\right), \quad \gamma_{0}\left(x_{0}, x_{0}\right)=\gamma_{0}\left(x_{1}\left(x_{0}\right), x_{0}\right)=0, \\
\frac{\partial \gamma_{0}}{\partial x}\left(x_{0}, x_{0}\right)>0, \quad \frac{\partial \gamma_{0}}{\partial x}\left(x_{1}\left(x_{0}\right), x_{0}\right)<0 .
\end{gathered}
$$

Объединяя, далее, свойства (3.51), (3.67), приходим к выводу о существовании у уравнения $u\left(x, x_{0}, \varepsilon\right)=\varepsilon^{1 / 4}$ такого корня $x=\overline{\bar{x}}\left(x_{0}, \varepsilon\right)$, что, во-первых, при $\varepsilon \rightarrow 0$ равномерно по $x_{0} \in[a, b]$ для него справедливы асимптотические равенства (3.49), (3.50); во-вторых, на интервале $\bar{x}\left(x_{0}, \varepsilon\right)<x<\overline{\bar{x}}\left(x_{0}, \varepsilon\right)$ имеет место требуемая в (3.46) оценка

$$
u\left(x, x_{0}, \varepsilon\right)>\varepsilon^{1 / 4} .
$$

Следует также добавить, что в силу неравенств

$$
\frac{a_{0}\left(x_{0}\right)}{b_{0}\left(x_{0}\right)}>0, \quad \frac{a_{0}\left(x_{1}\right)}{b_{0}\left(x_{1}\right)}<0
$$

и способа выбора постоянных $c_{1}, c_{2}$ (см. (3.61)) точки $x=\bar{x}\left(x_{0}, \varepsilon\right), x=\overline{\bar{x}}\left(x_{0}, \varepsilon\right)$ принадлежат отрезку (3.60).

Неравенство (3.68) и оценка $d<1$ (см. (3.59)) гарантируют выполнение условия (3.63) на множестве

$$
\left[\bar{x}\left(x_{0}, \varepsilon\right), \overline{\bar{x}}\left(x_{0}, \varepsilon\right)\right] \subset J .
$$

Более того, уменьшая, если это необходимо, константу $d$ в (3.59), можно добиться справедливости данного условия на всем отрезке $J$. Таким образом, при $x \in J$ обретают законную силу как сами равенства $(3.51)$, так и вытекающие из них соотношения (3.49), (3.50), (3.68).

Завершая обоснование леммы 3.3 , найдем оставшуюся функцию $\theta_{2}\left(x, x_{0}, \varepsilon\right)$. Как и в случае (3.24), она задается явным выражением

$$
\theta_{2}\left(x, x_{0}, \varepsilon\right)=\tau_{1}\left(x_{0}, \varepsilon\right)+\int_{\bar{x}\left(x_{0}, \varepsilon\right)}^{x} \frac{d s}{f\left(s, u\left(s, x_{0}, \varepsilon\right) / \varepsilon\right)} .
$$

Заметим далее, что в силу уже установленных формул (3.51) и свойств (2.1) функции $f(x, y)$ имеют место равномерные по $x_{0} \in[a, b], x \in J$ асимптотические представления

$$
\begin{gathered}
\frac{1}{f\left(x, u\left(x, x_{0}, \varepsilon\right) / \varepsilon\right)}=\frac{\varepsilon}{a_{0}(x) \gamma_{0}\left(x, x_{0}\right)}+O\left(\frac{\varepsilon^{3 / 2}}{\gamma_{0}^{2}\left(x, x_{0}\right)}\right) \\
\frac{\partial}{\partial x_{0}}\left(\frac{1}{f\left(x, u\left(x, x_{0}, \varepsilon\right) / \varepsilon\right)}\right)=\varepsilon \frac{\partial}{\partial x_{0}}\left(\frac{1}{a_{0}(x) \gamma_{0}\left(x, x_{0}\right)}\right)+O\left(\frac{\varepsilon^{3 / 2}}{\gamma_{0}^{3}\left(x, x_{0}\right)}\right) .
\end{gathered}
$$


Интегрируя затем указанные соотношения по $x$ и учитывая, что

$$
\begin{gathered}
\varepsilon^{3 / 2} \int_{\bar{x}\left(x_{0}, \varepsilon\right)}^{x} \frac{d s}{\gamma_{0}^{2}\left(s, x_{0}\right)}=O\left(\frac{\varepsilon^{3 / 2}}{\gamma_{0}\left(x, x_{0}\right)}\right)=O\left(\varepsilon^{5 / 4}\right), \\
\varepsilon^{3 / 2} \int_{\bar{x}\left(x_{0}, \varepsilon\right)}^{x} \frac{d s}{\gamma_{0}^{3}\left(s, x_{0}\right)}=O\left(\frac{\varepsilon^{3 / 2}}{\gamma_{0}^{2}\left(x, x_{0}\right)}\right), \quad \varepsilon^{3 / 2} \frac{\partial}{\partial x_{0}}\left(\int_{\bar{x}\left(x_{0}, \varepsilon\right)}^{x} \frac{d s}{\gamma_{0}^{2}\left(s, x_{0}\right)}\right)=O(\varepsilon),
\end{gathered}
$$

получаем нужные равенства (3.52)-(3.55). Лемма 3.3 доказана.

Переход от кривой $\Lambda_{2}(\varepsilon)$ к $\Gamma_{2}(\varepsilon)$ осуществляется по той же схеме, что и в случае участков взлета. Как будет показано ниже, эти кривые экспоненциально близки.

Лемма 3.4. Участок поворота $\Gamma_{2}(\varepsilon)$ имеет аналогичный (3.45) вид

$$
\Gamma_{2}(\varepsilon)=\left\{(t, x, y): t=\theta_{x_{0}, \varphi}^{(2)}(x, \varepsilon), y=\frac{u_{x_{0}, \varphi}(x, \varepsilon)}{\varepsilon}, \bar{x}_{x_{0}, \varphi}(\varepsilon) \leqslant x \leqslant \overline{\bar{x}}_{x_{0}, \varphi}(\varepsilon)\right\}
$$

где функции $u_{x_{0}, \varphi}(x, \varepsilon), \theta_{x_{0}, \varphi}^{(2)}(x, \varepsilon)$ обладают аналогичными (3.46) свойствами

$$
\begin{gathered}
\left.u_{x_{0}, \varphi}(x, \varepsilon)\right|_{x=\bar{x}_{x_{0}, \varphi}(\varepsilon), \overline{\bar{x}}_{x_{0}, \varphi}(\varepsilon)}=\varepsilon^{1 / 4}, \quad u_{x_{0}, \varphi}(x, \varepsilon)>\varepsilon^{1 / 4} \\
n p u \quad x \in\left(\bar{x}_{x_{0}, \varphi}(\varepsilon), \overline{\bar{x}}_{x_{0}, \varphi}(\varepsilon)\right),\left.\quad \theta_{x_{0}, \varphi}^{(2)}(x, \varepsilon)\right|_{x=\bar{x}_{x_{0}, \varphi}(\varepsilon)}=\tau_{\left(x_{0}, \varphi\right)}^{(1)}(\varepsilon) .
\end{gathered}
$$

Более того, при $\varepsilon \rightarrow 0$ для этих функиий справедливы равномерные по начальным данным $\left(x_{0}, \varphi\right) \in S$ и по $\bar{x}_{x_{0}, \varphi}(\varepsilon) \leqslant x \leqslant \overline{\bar{x}}_{x_{0}, \varphi}(\varepsilon)$ асимптотические представления

$$
\begin{gathered}
\left(u_{x_{0}, \varphi}(x, \varepsilon), \frac{\partial}{\partial x} u_{x_{0}, \varphi}(x, \varepsilon)\right)=\left(u\left(x, x_{0}, \varepsilon\right), \frac{\partial}{\partial x} u\left(x, x_{0}, \varepsilon\right)\right)+O\left(\exp \left(-\frac{M}{\varepsilon}\right)\right), \\
\left(\theta_{x_{0}, \varphi}^{(2)}(x, \varepsilon), \frac{\partial}{\partial x} \theta_{x_{0}, \varphi}^{(2)}(x, \varepsilon)\right)=\left(\theta_{2}\left(x, x_{0}, \varepsilon\right), \frac{\partial}{\partial x} \theta_{2}\left(x, x_{0}, \varepsilon\right)\right)+O\left(\exp \left(-\frac{M}{\varepsilon}\right)\right), \\
M=\mathrm{const}>0
\end{gathered}
$$

а для значений $x=\bar{x}_{x_{0}, \varphi}(\varepsilon), \overline{\bar{x}}_{x_{0}, \varphi}(\varepsilon)$ - равномерные по $\left(x_{0}, \varphi\right) \in S$ представления

$$
\begin{gathered}
\bar{x}_{x_{0}, \varphi}(\varepsilon)=\bar{x}\left(x_{0}, \varepsilon\right)+O\left(\exp \left(-\frac{M}{\varepsilon}\right)\right), \\
\overline{\bar{x}}_{x_{0}, \varphi}(\varepsilon)=\overline{\bar{x}}\left(x_{0}, \varepsilon\right)+O\left(\exp \left(-\frac{M}{\varepsilon}\right)\right), \quad M=\text { const }>0 .
\end{gathered}
$$

ДокАЗАТЕЛЬство. Как и при обосновании леммы 3.3, заметим сначала, что значение $x=\bar{x}_{x_{0}, \varphi}(\varepsilon)$ задается аналогичным (3.56) равенством

$$
\bar{x}_{x_{0}, \varphi}(\varepsilon)=\left.\widetilde{x}_{x_{0}, \varphi}(y, \varepsilon)\right|_{y=\varepsilon^{-3 / 4}}
$$

А отсюда и из (3.26), (3.56) первое соотношение (3.73) вытекает автоматически. 
Перейдем теперь к отысканию фигурирующих в выражениях (3.70), (3.71) функций $u_{x_{0}, \varphi}(x, \varepsilon), \theta_{x_{0}, \varphi}^{(2)}(x, \varepsilon)$. С этой целью предположим, что на кривой $\Gamma_{2}(\varepsilon)$ переменные $t, x$ связаны формулой

$$
t=\theta(x)
$$

где $\theta(x)$ - пока произвольная функция, определенная на отрезке $J$ (см. (3.60), (3.61)) и принадлежащая множеству

$$
\Omega=\left\{\theta(x) \in C(J): 0 \leqslant \theta(x) \leqslant M \varepsilon \ln \frac{1}{\varepsilon},\left.\theta\right|_{x=\bar{x}_{x_{0}, \varphi}(\varepsilon)}=\tau_{\left(x_{0}, \varphi\right)}^{(1)}(\varepsilon)\right\} .
$$

Здесь, как и в (3.28), $M>0$ - универсальная константа, выбором которой распорядимся в дальнейшем.

Подставим соотношение (3.75) в правые части системы из (3.4) и выполним в ней замену $y=u / \varepsilon$. В результате для отыскания переменной $u$ как функции от $x$ получим аналогичную (3.58) задачу Коши

$$
\frac{d u}{d x}=\frac{b_{0}(x)}{a_{0}(x)}+\Phi(u, x, \theta(x), \varepsilon),\left.\quad u\right|_{x=\bar{x}_{x_{0}, \varphi}(\varepsilon)}=\varepsilon^{1 / 4} .
$$

Как и выше, явная формула для функции $\Phi(u, x, \theta, \varepsilon)$ нам не понадобится. Заметим лишь, что $\forall(x, u) \in \Sigma, \forall \theta \in \Omega$, где $\Sigma, \Omega$ - множества (3.59), (3.76), справедливы аналогичные (3.62) оценки

$$
\begin{gathered}
|\Phi(u, x, \theta(x), \varepsilon)| \leqslant M_{1} \varepsilon^{3 / 4}, \quad\left|\Phi_{u}^{\prime}(u, x, \theta(x), \varepsilon)\right| \leqslant M_{2} \sqrt{\varepsilon}, \\
|\Phi(u, x, \theta(x), \varepsilon)-\Phi(u, x, \varepsilon)| \leqslant M_{3} \exp \left(-\frac{M_{4}}{\varepsilon}\right),
\end{gathered}
$$

где $\Phi(u, x, \varepsilon)$ - функция из (3.58).

Неравенства (3.78) и уже известная асимптотическая формула для $\bar{x}_{x_{0}, \varphi}(\varepsilon)$ (см. (3.73)) позволяют применить при исследовании задачи Коши (3.77) ту же методику, что и в случае задачи (3.58). Таким образом, повторяя практически дословно соответствующий фрагмент доказательства леммы 3.3, получаем следующие факты.

Во-первых, решение $u_{x_{0}, \varphi, \theta}(x, \varepsilon)$ задачи Коши $(3.77)$ при $\varepsilon \rightarrow 0$ допускает равномерную по $\left(x_{0}, \varphi\right) \in S, \theta \in \Omega, x \in J$ асимптотику

$$
\left(u_{x_{0}, \varphi, \theta}(x, \varepsilon), \frac{\partial}{\partial x} u_{x_{0}, \varphi, \theta}(x, \varepsilon)\right)=\left(u\left(x, x_{0}, \varepsilon\right), \frac{\partial}{\partial x} u\left(x, x_{0}, \varepsilon\right)\right)+O\left(\exp \left(-\frac{M}{\varepsilon}\right)\right),
$$

где $M=$ const $>0$; во-вторых, уравнение $u_{x_{0}, \varphi, \theta}(x, \varepsilon)=\varepsilon^{1 / 4}$ имеет такой корень $x=\overline{\bar{x}}_{x_{0}, \varphi, \theta}(\varepsilon)$, что равномерно по $\left(x_{0}, \varphi\right) \in S, \theta \in \Omega$

$$
\overline{\bar{x}}_{x_{0}, \varphi, \theta}(\varepsilon)=\overline{\bar{x}}\left(x_{0}, \varepsilon\right)+O\left(\exp \left(-\frac{M}{\varepsilon}\right)\right), \quad \varepsilon \rightarrow 0, \quad M=\text { const }>0
$$

и $u_{x_{0}, \varphi, \theta}(x, \varepsilon)>\varepsilon^{1 / 4}$ при $x \in\left(\bar{x}_{x_{0}, \varphi}(\varepsilon), \overline{\bar{x}}_{x_{0}, \varphi, \theta}(\varepsilon)\right)$. 
Заключительный этап доказательства леммы 3.4 связан с нахождением функции $\theta(x)$ из (3.75). Обращаясь в очередной раз к системе (3.4), убеждаемся, что $\theta(x)$ с необходимостью удовлетворяет интегральному уравнению

$$
\theta(x)=\mathscr{L}_{2}(\theta)(x) \stackrel{\text { def }}{=} \tau_{\left(x_{0}, \varphi\right)}^{(1)}(\varepsilon)+\int_{\bar{x}_{x_{0}, \varphi}(\varepsilon)}^{x} \frac{d s}{f\left(s, u_{x_{0}, \varphi, \theta}(s, \varepsilon) / \varepsilon\right)} .
$$

Свойства (2.1) функции $f(x, y)$, формула (3.69) и уже известные асимптотические равенства $(3.44),(3.73)$ (для $\left.\bar{x}_{x_{0}, \varphi}(\varepsilon)\right),(3.79)$ позволяют заключить, что равномерно по $x \in J, \theta(x) \in \Omega,\left(x_{0}, \varphi\right) \in S$

$$
\begin{gathered}
\left(\mathscr{L}_{2}(\theta)(x), \frac{\partial}{\partial x} \mathscr{L}_{2}(\theta)(x)\right)=\left(\theta_{2}\left(x, x_{0}, \varepsilon\right), \frac{\partial}{\partial x} \theta_{2}\left(x, x_{0}, \varepsilon\right)\right)+O\left(\exp \left(-\frac{M}{\varepsilon}\right)\right), \\
\varepsilon \rightarrow 0,
\end{gathered}
$$

где $M=$ const $>0$. А отсюда и из (3.42)-(3.44), (3.52) вытекает, что

$$
\max _{x \in J}\left|\mathscr{L}_{2}(\theta)(x)\right|=\left(\frac{1}{b_{0}\left(x_{0}\right)}-\frac{1}{4 b_{0}\left(x_{1}\left(x_{0}\right)\right)}\right) \varepsilon \ln \frac{1}{\varepsilon}+O(\varepsilon)
$$

(при выводе этой формулы использовалась процедура регуляризации по Адамару интеграла

$$
\int \frac{d x}{a_{0}(x) \gamma_{0}\left(x, x_{0}\right)}
$$

в точках $x=x_{0}$ и $\left.x=x_{1}\left(x_{0}\right)\right)$.

Из установленных соотношений (3.82), (3.83) следует, что фигурирующий в (3.81) компактный оператор $\mathscr{L}_{2}$ при подходящем выборе постоянной $M$ в (3.76), а точнее говоря, при

$$
M>\frac{1}{b_{0}\left(x_{0}\right)}-\frac{1}{4 b_{0}\left(x_{1}\left(x_{0}\right)\right)},
$$

переводит замкнутое ограниченное выпуклое множество $\Omega \subset C(J)$ в себя. Тем самым, он имеет в $\Omega$ хотя бы одну неподвижную точку

$$
\begin{aligned}
\theta=\theta_{x_{0}, \varphi}^{(2)}(x, \varepsilon), \\
\left(\theta_{x_{0}, \varphi}^{(2)}(x, \varepsilon), \frac{\partial}{\partial x} \theta_{x_{0}, \varphi}^{(2)}(x, \varepsilon)\right)=\left(\theta_{2}\left(x, x_{0}, \varepsilon\right), \frac{\partial}{\partial x} \theta_{2}\left(x, x_{0}, \varepsilon\right)\right)+O\left(\exp \left(-\frac{M}{\varepsilon}\right)\right), \\
\varepsilon \rightarrow 0, \quad M=\text { const }>0 .
\end{aligned}
$$

Добавим еще, что на самом деле эта неподвижная точка единственна, так как единственна функция $x_{\left(x_{0}, \varphi\right)}(t, \varepsilon)$, определяющаяся как неявная из уравнения $t=\theta_{x_{0}, \varphi}^{(2)}(x, \varepsilon)$.

На завершающем этапе положим

$$
u_{x_{0}, \varphi}(x, \varepsilon)=\left.u_{x_{0}, \varphi, \theta}(x, \varepsilon)\right|_{\theta=\theta_{x_{0}, \varphi}^{(2)}}, \quad \overline{\bar{x}}_{x_{0}, \varphi}(\varepsilon)=\left.\overline{\bar{x}}_{x_{0}, \varphi, \theta}(\varepsilon)\right|_{\theta=\theta_{x_{0}, \varphi}^{(2)}}
$$

и заметим, что получившийся в итоге набор функций (3.74), (3.84), (3.85) удовлетворяет всем требованиям (3.71)-(3.73). Лемма 3.4 доказана. 
Заканчивая исследование участков поворота $\Gamma_{2}(\varepsilon), \Lambda_{2}(\varepsilon)$, отметим, что вторые положительные корни $\tau_{\left(x_{0}, \varphi\right)}^{(2)}(\varepsilon), \tau_{2}\left(x_{0}, \varepsilon\right)$ уравнений $(3.10)$, о которых говорилось выше, в силу равенств (3.42)-(3.44), (3.47)-(3.50), (3.52), (3.53), (3.72), (3.73) допускают равномерные по $\left(x_{0}, \varphi\right) \in S$ асимптотические представления

$$
\begin{gathered}
\left.\tau_{2}\left(x_{0}, \varepsilon\right) \stackrel{\text { def }}{=} \theta_{2}\left(x, x_{0}, \varepsilon\right)\right|_{x=\overline{\bar{x}}\left(x_{0}, \varepsilon\right)}=\left(\frac{1}{b_{0}\left(x_{0}\right)}-\frac{1}{4 b_{0}\left(x_{1}\left(x_{0}\right)\right)}\right) \varepsilon \ln \frac{1}{\varepsilon}+O(\varepsilon), \\
\frac{\partial}{\partial x_{0}} \tau_{2}\left(x_{0}, \varepsilon\right)=\frac{\partial}{\partial x_{0}}\left[\left(\frac{1}{b_{0}\left(x_{0}\right)}-\frac{1}{4 b_{0}\left(x_{1}\left(x_{0}\right)\right)}\right) \varepsilon \ln \frac{1}{\varepsilon}\right]+O(\varepsilon) \\
\left.\tau_{\left(x_{0}, \varphi\right)}^{(2)}(\varepsilon) \stackrel{\text { def }}{=} \theta_{x_{0}, \varphi}^{(2)}(x, \varepsilon)\right|_{x=\bar{x}_{x_{0}, \varphi}(\varepsilon)}=\tau_{2}\left(x_{0}, \varepsilon\right)+O\left(\exp \left(-\frac{M}{\varepsilon}\right)\right) \\
M=\text { const }>0 .
\end{gathered}
$$

Отметим еще, что проекция участка $\Gamma_{2}(\varepsilon)$ на плоскость $(x, u)$, где $u=\varepsilon y$, при $\varepsilon \rightarrow 0$ стремится к кривой

$$
\Gamma_{2}^{0}=\left\{(x, u): u=\gamma_{0}\left(x, x_{0}\right), x_{0} \leqslant x \leqslant x_{1}\left(x_{0}\right)\right\}
$$

График этой кривой аналогичен показанному на рис. 2 (необходимо поменять местами точки $x_{0}, x_{1}$ и сменить направление движения).

Следующие участки $\Gamma_{3}(\varepsilon), \Lambda_{3}(\varepsilon)$ кривых (3.8), (3.9) соответствуют значениям $t$ из отрезков

$$
\left[\tau_{\left(x_{0}, \varphi\right)}^{(2)}(\varepsilon), \tau_{\left(x_{0}, \varphi\right)}^{(3)}(\varepsilon)\right], \quad\left[\tau_{2}\left(x_{0}, \varepsilon\right), \tau_{3}\left(x_{0}, \varepsilon\right)\right]
$$

где $\tau_{\left(x_{0}, \varphi\right)}^{(3)}(\varepsilon), \tau_{3}\left(x_{0}, \varepsilon\right)$ - первые положительные корни уравнений

$$
y_{\left(x_{0}, \varphi\right)}(t, \varepsilon)=\exp \left(-\varepsilon^{-3 / 4}\right), \quad y\left(t, x_{0}, \varepsilon\right)=\exp \left(-\varepsilon^{-3 / 4}\right) .
$$

В дальнейшем будет показано, что длины данных отрезков асимптотически малы, причем за это время компоненты $y_{\left(x_{0}, \varphi\right)}(t, \varepsilon), y\left(t, x_{0}, \varepsilon\right)$ монотонно убывают от $\varepsilon^{-3 / 4}$ до асимптотически малого значения $\exp \left(-\varepsilon^{-3 / 4}\right)$ (см. рис. 5). Тем самым, участки $\Gamma_{3}(\varepsilon), \Lambda_{3}(\varepsilon)$ уместно назвать участками падения. Как и в случае $\Gamma_{1}(\varepsilon), \Lambda_{1}(\varepsilon)$, в качестве параметра на них возьмем переменную $y$.

Лемма 3.5. Для участка падения $\Lambda_{3}(\varepsilon)$ справедливо аналогичное (3.11) представление

$$
\Lambda_{3}(\varepsilon)=\left\{(t, x, y): t=\theta_{3}\left(y, x_{0}, \varepsilon\right), x=\widetilde{\widetilde{x}}\left(y, x_{0}, \varepsilon\right), \exp \left(-\varepsilon^{-3 / 4}\right) \leqslant y \leqslant \varepsilon^{-3 / 4}\right\} .
$$

Здесъ функиии $\theta_{3}\left(y, x_{0}, \varepsilon\right), \widetilde{\widetilde{x}}\left(y, x_{0}, \varepsilon\right)$ таковы, что, во-первых,

$$
\left.\theta_{3}\left(y, x_{0}, \varepsilon\right)\right|_{y=\varepsilon^{-3 / 4}}=\tau_{2}\left(x_{0}, \varepsilon\right),\left.\quad \widetilde{\widetilde{x}}\left(y, x_{0}, \varepsilon\right)\right|_{y=\varepsilon^{-3 / 4}}=\overline{\bar{x}}\left(x_{0}, \varepsilon\right)
$$


во-вторых, при $\varepsilon \rightarrow 0$ равномерно по $x_{0} \in[a, b], y \in\left[\exp \left(-\varepsilon^{-3 / 4}\right), \varepsilon^{-3 / 4}\right]$ выполняются асимптотические равенства

$$
\begin{gathered}
\theta_{3}\left(y, x_{0}, \varepsilon\right)=\tau_{2}\left(x_{0}, \varepsilon\right)+\varepsilon \int_{\varepsilon^{-3 / 4}}^{y} \frac{d s}{s g\left(x_{1}\left(x_{0}\right), s\right)}+O\left(\varepsilon^{5 / 4} \ln \frac{\varepsilon^{-3 / 4}}{y}\right) \\
\frac{\partial}{\partial x_{0}} \theta_{3}\left(y, x_{0}, \varepsilon\right)=\frac{\partial}{\partial x_{0}}\left(\tau_{2}\left(x_{0}, \varepsilon\right)+\varepsilon \int_{\varepsilon^{-3 / 4}}^{y} \frac{d s}{s g\left(x_{1}\left(x_{0}\right), s\right)}\right)+O\left(\varepsilon^{5 / 4} \ln \frac{\varepsilon^{-3 / 4}}{y}\right), \\
\frac{\partial}{\partial y} \theta_{3}\left(y, x_{0}, \varepsilon\right)=\frac{\varepsilon}{y}\left(\frac{1}{g\left(x_{1}\left(x_{0}\right), y\right)}+O\left(\varepsilon^{1 / 4}\right)\right) \\
\frac{\partial^{2}}{\partial x_{0} \partial y} \theta_{3}\left(y, x_{0}, \varepsilon\right)=\frac{\varepsilon}{y}\left(\frac{\partial}{\partial x_{0}}\left(\frac{1}{g\left(x_{1}\left(x_{0}\right), y\right)}\right)+O\left(\varepsilon^{1 / 4}\right)\right) \\
\widetilde{x}\left(y, x_{0}, \varepsilon\right)=x_{1}\left(x_{0}\right)+O\left(\varepsilon^{1 / 4}\right), \frac{\partial}{\partial y} \widetilde{\widetilde{x}}\left(y, x_{0}, \varepsilon\right)=O\left(\varepsilon \frac{y+1}{y}\right) \\
\frac{\partial}{\partial x_{0}} \widetilde{\widetilde{x}}\left(y, x_{0}, \varepsilon\right)=x_{1}^{\prime}\left(x_{0}\right)+O\left(\varepsilon^{1 / 4}\right) .
\end{gathered}
$$

ДокАЗАТЕльство. Поскольку обоснование леммы 3.5 во многом аналогично изложенному выше доказательству леммы 3.1, то здесь опустим ряд технических деталей.

В данном случае для определения фигурирующей в (3.90), (3.91) функции $x=\widetilde{\widetilde{x}}\left(y, x_{0}, \varepsilon\right)$ имеем дело с аналогичной (3.17) задачей Коши

$$
\frac{d x}{d y}=\varepsilon F(x, y),\left.\quad x\right|_{y=\varepsilon^{-3 / 4}}=\overline{\bar{x}}\left(x_{0}, \varepsilon\right),
$$

где $F(x, y)$ - функция (3.18). При ее исследовании нам потребуется аналогичное (3.20) множество

$$
\Sigma=\left\{(x, y): x_{1}\left(b^{\prime}\right) \leqslant x \leqslant x_{1}\left(a^{\prime}\right), \exp \left(-\varepsilon^{-3 / 4}\right) \leqslant y \leqslant \varepsilon^{-3 / 4}\right\},
$$

где постоянные $a^{\prime}<a, b^{\prime}>b$ выбраны настолько близкими к $a, b$, что при $x_{1}\left(b^{\prime}\right) \leqslant x \leqslant x_{1}\left(a^{\prime}\right)$ сохраняется второе неравенство (3.3), т.е.

$$
g(x, y)<0 \quad \forall y \geqslant 0, \quad \forall x \in\left[x_{1}\left(b^{\prime}\right), x_{1}\left(a^{\prime}\right)\right] .
$$

Объединяя, далее, свойство (3.99) с (2.1)-(2.3), здесь вместо (3.21) получаем оценки вида

$$
\begin{gathered}
|F(x, y)| \leqslant M_{1} \frac{y+1}{y}, \quad\left|F_{x}^{\prime}(x, y)\right| \leqslant M_{2} \frac{y+1}{y}, \quad\left|F_{x x}^{\prime \prime}(x, y)\right| \leqslant M_{3} \frac{y+1}{y} \\
\forall(x, y) \in \Sigma .
\end{gathered}
$$

Последующие рассуждения повторяют соответствующий фрагмент доказательства леммы 3.1. А именно, в данном случае через $X$ обозначаем банахово пространство функций $x\left(y, x_{0}\right)$, непрерывных по совокупности переменных 
$\left(y, x_{0}\right) \in\left[\exp \left(-\varepsilon^{-3 / 4}\right), \varepsilon^{-3 / 4}\right] \times[a, b]$ вместе с частной производной $\partial x / \partial x_{0}, \mathrm{a}$ вместо (3.22) рассматриваем оператор

$$
\mathscr{L}_{0}(x)\left(y, x_{0}\right)=\overline{\bar{x}}\left(x_{0}, \varepsilon\right)+\varepsilon \int_{\varepsilon^{-3 / 4}}^{y} F\left(x\left(s, x_{0}\right), s\right) d s .
$$

Опираясь, далее, на оценки (3.100) и формулы (3.49), (3.50), убеждаемся, что, во-первых, этот оператор переводит аналогичное (3.23) множество функций

$$
\left\{x\left(y, x_{0}\right): x_{1}\left(b^{\prime}\right) \leqslant x\left(y, x_{0}\right) \leqslant x_{1}\left(a^{\prime}\right),\left|\frac{\partial x}{\partial x_{0}}\left(y, x_{0}\right)\right| \leqslant 2 \max _{a \leqslant x_{0} \leqslant b}\left|x_{1}^{\prime}\left(x_{0}\right)\right|\right\} \subset X
$$

в себя и является сжимающим; во-вторых, для его неподвижной точки $x=$ $\widetilde{\widetilde{x}}\left(y, x_{0}, \varepsilon\right)$ имеют место равномерные по $x_{0} \in[a, b], y \in\left[\exp \left(-\varepsilon^{-3 / 4}\right), \varepsilon^{-3 / 4}\right]$ асимптотические равенства

$$
\begin{aligned}
\widetilde{\widetilde{x}}\left(y, x_{0}, \varepsilon\right)=\overline{\bar{x}}\left(x_{0}, \varepsilon\right)+ & \varepsilon \int_{\varepsilon^{-3 / 4}}^{y} \frac{f\left(x_{1}\left(x_{0}\right), s\right)}{s g\left(x_{1}\left(x_{0}\right), s\right)} d s+O\left(\varepsilon^{5 / 4} \int_{y}^{\varepsilon^{-3 / 4}} \frac{s+1}{s} d s\right), \\
\frac{\partial}{\partial x_{0}} \widetilde{\widetilde{x}}\left(y, x_{0}, \varepsilon\right)= & \frac{\partial}{\partial x_{0}}\left(\overline{\bar{x}}\left(x_{0}, \varepsilon\right)+\varepsilon \int_{\varepsilon^{-3 / 4}}^{y} \frac{f\left(x_{1}\left(x_{0}\right), s\right)}{s g\left(x_{1}\left(x_{0}\right), s\right)} d s\right) \\
& +O\left(\varepsilon^{5 / 4} \int_{y}^{\varepsilon^{-3 / 4}} \frac{s+1}{s} d s\right)
\end{aligned}
$$

из которых в силу (2.1), (3.49), (3.50) вытекают требуемые формулы (3.96). Что же касается оставшихся равенств (3.92)-(3.95), то они получаются из соответствующего явного выражения

$$
\theta_{3}\left(y, x_{0}, \varepsilon\right)=\tau_{2}\left(x_{0}, \varepsilon\right)+\varepsilon \int_{\varepsilon^{-3 / 4}}^{y} \frac{d s}{s g\left(\widetilde{\widetilde{x}}\left(s, x_{0}, \varepsilon\right), s\right)}
$$

с учетом уже известных фактов (3.86), (3.87), (3.96). Лемма 3.5 доказана.

Покажем теперь, что, как и в предыдущих случаях, участки $\Lambda_{3}(\varepsilon)$ и $\Gamma_{3}(\varepsilon)$ экспоненциально близки.

ЛЕмма 3.6. Участок $\Gamma_{3}(\varepsilon)$ имеет вид

$$
\Gamma_{3}(\varepsilon)=\left\{(t, x, y): t=\theta_{x_{0}, \varphi}^{(3)}(y, \varepsilon), x=\widetilde{\widetilde{x}}_{x_{0}, \varphi}(y, \varepsilon), \exp \left(-\varepsilon^{-3 / 4}\right) \leqslant y \leqslant \varepsilon^{-3 / 4}\right\},
$$

¿əe

$$
\left.\theta_{x_{0}, \varphi}^{(3)}(y, \varepsilon)\right|_{y=\varepsilon^{-3 / 4}}=\tau_{\left(x_{0}, \varphi\right)}^{(2)}(\varepsilon),\left.\quad \widetilde{\widetilde{x}}_{x_{0}, \varphi}(y, \varepsilon)\right|_{y=\varepsilon^{-3 / 4}}=\overline{\bar{x}}_{x_{0}, \varphi}(\varepsilon)
$$

и равномерно по $\left(x_{0}, \varphi\right) \in S, y \in\left[\exp \left(-\varepsilon^{-3 / 4}\right), \varepsilon^{-3 / 4}\right]$

$$
\begin{gathered}
\left(\widetilde{\widetilde{x}}_{x_{0}, \varphi}(y, \varepsilon), \frac{\partial}{\partial y} \widetilde{\widetilde{x}}_{x_{0}, \varphi}(y, \varepsilon)\right)=\left(\widetilde{\widetilde{x}}\left(y, x_{0}, \varepsilon\right), \frac{\partial}{\partial y} \widetilde{\widetilde{x}}\left(y, x_{0}, \varepsilon\right)\right)+O\left(\exp \left(-\frac{M}{\varepsilon}\right)\right), \\
\left(\theta_{x_{0}, \varphi}^{(3)}(y, \varepsilon), \frac{\partial}{\partial y} \theta_{x_{0}, \varphi}^{(3)}(y, \varepsilon)\right)=\left(\theta_{3}\left(y, x_{0}, \varepsilon\right), \frac{\partial}{\partial y} \theta_{3}\left(y, x_{0}, \varepsilon\right)\right)+O\left(\exp \left(-\frac{M}{\varepsilon}\right)\right), \\
M=\text { const }>0 .
\end{gathered}
$$


ДокАЗАТЕЛЬСтво. Обоснование сформулированной леммы проводится по той же схеме, что и доказательство леммы 3.2. Поэтому имеет смысл опустить некоторые детали технического характера.

Как и в случае участка $\Gamma_{1}(\varepsilon)$, будем считать, что переменные $t, y$ на $\Gamma_{3}(\varepsilon)$ связаны соотношением (3.27), где функция $\theta(y)$ принадлежит аналогичному (3.28) множеству

$$
\Omega=\left\{\theta(y) \in C\left[\exp \left(-\varepsilon^{-3 / 4}\right), \varepsilon^{-3 / 4}\right]: 0 \leqslant \theta(y) \leqslant M \varepsilon^{1 / 4},\left.\theta\right|_{y=\varepsilon^{-3 / 4}}=\tau_{\left(x_{0}, \varphi\right)}^{(2)}(\varepsilon)\right\} .
$$

В этом случае переменная $x$, рассматриваемая как функция от $y$, удовлетворяет аналогичной (3.29) задаче Коши

$$
\frac{d x}{d y}=\varepsilon F(x, y, \theta(y), \varepsilon),\left.\quad x\right|_{y=\varepsilon^{-3 / 4}}=\overline{\bar{x}}_{x_{0}, \varphi}(\varepsilon),
$$

где $F(x, y, \theta, \varepsilon)$ - функция (3.30). Сохраняются здесь и аналогичные (3.31), (3.100) оценки

$$
\begin{gathered}
|F(x, y, \theta(y), \varepsilon)| \leqslant M_{1} \frac{y+1}{y}, \quad\left|F_{x}^{\prime}(x, y, \theta(y), \varepsilon)\right| \leqslant M_{2} \frac{y+1}{y}, \\
|F(x, y, \theta(y), \varepsilon)-F(x, y)| \leqslant M_{3} \frac{y+1}{y} \exp \left(-\frac{M_{4}}{\varepsilon}\right)
\end{gathered}
$$

справедливые $\forall(x, y) \in \Sigma, \forall \theta(y) \in \Omega$, где $\Sigma, \Omega$ - множества (3.98), (3.107).

Последующий ход рассуждений стандартен: вводим в рассмотрение аналогичный (3.32) оператор

$$
\mathscr{L}_{0}(x)(y)=\overline{\bar{x}}_{x_{0}, \varphi}(\varepsilon)+\varepsilon \int_{\varepsilon^{-3 / 4}}^{y} F(x(s), s, \theta(s), \varepsilon) d s
$$

и, опираясь на неравенства (3.109), убеждаемся, что на аналогичном (3.33) множестве функций

$$
\left\{x(y): x_{1}\left(b^{\prime}\right) \leqslant x(y) \leqslant x_{1}\left(a^{\prime}\right)\right\} \subset C\left[\exp \left(-\varepsilon^{-3 / 4}\right), \varepsilon^{-3 / 4}\right]
$$

он удовлетворяет условиям принципа сжимающих отображений. Кроме того, нетрудно показать, что для его неподвижной точки $x=x_{x_{0}, \varphi, \theta}(y, \varepsilon)$ выполняются аналогичные (3.34), (3.35) оценки

$$
\begin{aligned}
& \left|\frac{\partial}{\partial y} x_{x_{0}, \varphi, \theta}(y, \varepsilon)-\frac{\partial}{\partial y} \widetilde{\widetilde{x}}\left(y, x_{0}, \varepsilon\right)\right| \\
& \leqslant \varepsilon M_{2} \frac{y+1}{y}\left|x_{x_{0}, \varphi, \theta}(y, \varepsilon)-\widetilde{\widetilde{x}}\left(y, x_{0}, \varepsilon\right)\right|+\varepsilon M_{3} \frac{y+1}{y} \exp \left(-\frac{M_{4}}{\varepsilon}\right), \\
& \left|x_{x_{0}, \varphi, \theta}(y, \varepsilon)-\widetilde{\widetilde{x}}\left(y, x_{0}, \varepsilon\right)\right| \\
& \leqslant\left|\overline{\bar{x}}_{x_{0}, \varphi}(\varepsilon)-\overline{\bar{x}}\left(x_{0}, \varepsilon\right)\right|+\varepsilon M_{2} \int_{y}^{\varepsilon^{-3 / 4}} \frac{s+1}{s}\left|x_{x_{0}, \varphi, \theta}(s, \varepsilon)-\widetilde{\widetilde{x}}\left(s, x_{0}, \varepsilon\right)\right| d s \\
& \quad+\varepsilon M_{3} \int_{y}^{\varepsilon^{-3 / 4}} \frac{s+1}{s} d s \exp \left(-\frac{M_{4}}{\varepsilon}\right)
\end{aligned}
$$


где $\widetilde{\widetilde{x}}\left(y, x_{0}, \varepsilon\right)$ - функция из (3.90). Учитывая, далее, в (3.110), (3.111) второе соотношение (3.73), приходим к аналогичному (3.36) неравенству

$$
\begin{gathered}
\left|x_{x_{0}, \varphi, \theta}(y, \varepsilon)-\widetilde{\widetilde{x}}\left(y, x_{0}, \varepsilon\right)\right|+\left|\frac{\partial}{\partial y} x_{x_{0}, \varphi, \theta}(y, \varepsilon)-\frac{\partial}{\partial y} \widetilde{\widetilde{x}}\left(y, x_{0}, \varepsilon\right)\right| \leqslant \exp \left(-\frac{M}{\varepsilon}\right), \\
M=\mathrm{const}>0, \quad y \in\left[\exp \left(-\varepsilon^{-3 / 4}\right), \varepsilon^{-3 / 4}\right] .
\end{gathered}
$$

Вопрос об отыскании функции $\theta(y)$ связан с рассмотрением аналогичного (3.37) интегрального уравнения

$$
\theta(y)=\mathscr{L}_{1}(\theta)(y) \stackrel{\text { def }}{=} \tau_{\left(x_{0}, \varphi\right)}^{(2)}(\varepsilon)+\varepsilon \int_{\varepsilon^{-3 / 4}}^{y} \frac{1}{s} G\left(x_{x_{0}, \varphi, \theta}(s, \varepsilon), s, \theta(s), \varepsilon\right) d s,
$$

где $G(x, y, \theta, \varepsilon)$ - функция (3.38), обладающая прежними свойствами (3.39) (в которых теперь $\Sigma, \Omega$ - множества (3.98), (3.107)). Сохраняется здесь и аналогичная (3.40) оценка

$$
\begin{gathered}
\left|G\left(x_{x_{0}, \varphi, \theta}(y, \varepsilon), y, \theta(y), \varepsilon\right)-\frac{1}{g\left(\widetilde{\widetilde{x}}\left(y, x_{0}, \varepsilon\right), y\right)}\right| \leqslant M_{2}\left|x_{x_{0}, \varphi, \theta}(y, \varepsilon)-\widetilde{\widetilde{x}}\left(y, x_{0}, \varepsilon\right)\right| \\
+M_{3} \exp \left(-\frac{M_{4}}{\varepsilon}\right) \quad \forall y \in\left[\exp \left(-\varepsilon^{-3 / 4}\right), \varepsilon^{-3 / 4}\right],
\end{gathered}
$$

из которой с учетом соотношений $(3.88),(3.103),(3.112)$ получаем аналогичные (3.41) равномерные по $y \in\left[\exp \left(-\varepsilon^{-3 / 4}\right), \varepsilon^{-3 / 4}\right], \theta(y) \in \Omega,\left(x_{0}, \varphi\right) \in S$ асимптотические представления

$$
\begin{gathered}
\mathscr{L}_{1}(\theta)(y)=\theta_{3}\left(y, x_{0}, \varepsilon\right)+O\left(\exp \left(-\frac{M}{\varepsilon}\right)\right) \\
\frac{\partial}{\partial y} \mathscr{L}_{1}(\theta)(y)=\frac{\partial}{\partial y} \theta_{3}\left(y, x_{0}, \varepsilon\right)+O\left(\exp \left(-\frac{M}{\varepsilon}\right)\right), \quad \varepsilon \rightarrow 0, \quad M=\text { const }>0 .
\end{gathered}
$$

Заключительный блок рассуждений повторяет соответствующее место из доказательства леммы 3.2. А именно, отталкиваясь от формул (3.113), нетрудно проверить, что оператор $\mathscr{L}_{1}$ при подходящем выборе константы $M$ в (3.107) преобразует множество $\Omega$ в себя, а его неподвижная точка $\theta_{x_{0}, \varphi}^{(3)}(y, \varepsilon)$, существующая в силу принципа Шаудера, на самом деле единственна и обладает требуемым свойством из (3.106). Добавим еще, что функция $\widetilde{\widetilde{x}}_{x_{0}, \varphi}(y, \varepsilon)$ из формул (3.104), (3.105), как и в лемме 3.2, задается соотношением

$$
\widetilde{\widetilde{x}}_{x_{0}, \varphi}(y, \varepsilon)=\left.x_{x_{0}, \varphi, \theta}(y, \varepsilon)\right|_{\theta=\theta_{x_{0}, \varphi}^{(3)}}
$$

и в силу (3.112) также удовлетворяет соответствующему асимптотическому равенству из (3.106). Лемма 3.6 доказана.

Как и в двух предыдущих случаях, отдельного рассмотрения требует вопрос об асимптотике очередных моментов времени $\tau_{\left(x_{0}, \varphi\right)}^{(3)}(\varepsilon), \tau_{3}\left(x_{0}, \varepsilon\right)$, которые, напомним, являются первыми положительными корнями уравнений (3.89). Из 
соотношений (3.86), (3.87), (3.92), (3.93), (3.106) следует, что при $\varepsilon \rightarrow 0$ равномерно по $\left(x_{0}, \varphi\right) \in S$

$$
\begin{gathered}
\left.\tau_{3}\left(x_{0}, \varepsilon\right) \stackrel{\text { def }}{=} \theta_{3}\left(y, x_{0}, \varepsilon\right)\right|_{y=\exp \left(-\varepsilon^{-3 / 4}\right)}=-\frac{\varepsilon^{1 / 4}}{g\left(x_{1}\left(x_{0}\right), 0\right)}+O(\sqrt{\varepsilon}), \\
\frac{\partial}{\partial x_{0}} \tau_{3}\left(x_{0}, \varepsilon\right)=-\frac{\partial}{\partial x_{0}}\left(\frac{\varepsilon^{1 / 4}}{g\left(x_{1}\left(x_{0}\right), 0\right)}\right)+O(\sqrt{\varepsilon}), \\
\left.\tau_{\left(x_{0}, \varphi\right)}^{(3)}(\varepsilon) \stackrel{\text { def }}{=} \theta_{x_{0}, \varphi}^{(3)}(y, \varepsilon)\right|_{y=\exp \left(-\varepsilon^{-3 / 4}\right)}=\tau_{3}\left(x_{0}, \varepsilon\right)+O\left(\exp \left(-\frac{M}{\varepsilon}\right)\right), \\
M=\text { const }>0 .
\end{gathered}
$$

Кроме того, теперь мы можем найти и первый положительный корень $t_{\left(x_{0}, \varphi\right)}^{(1)}(\varepsilon)$ уравнения (2.27). Этот корень задается равенством

$$
t_{\left(x_{0}, \varphi\right)}^{(1)}(\varepsilon)=\left.\theta_{x_{0}, \varphi}^{(3)}(y, \varepsilon)\right|_{y=1}
$$

и в силу $(3.86),(3.92),(3.106)$ допускает при $\varepsilon \rightarrow 0$ равномерное по $\left(x_{0}, \varphi\right) \in S$ асимптотическое представление

$$
t_{\left(x_{0}, \varphi\right)}^{(1)}(\varepsilon)=\left(\frac{1}{b_{0}\left(x_{0}\right)}-\frac{1}{b_{0}\left(x_{1}\left(x_{0}\right)\right)}\right) \varepsilon \ln \frac{1}{\varepsilon}+O(\varepsilon) .
$$

Оставшиеся участки $\Gamma_{4}(\varepsilon), \Lambda_{4}(\varepsilon)$ кривых (3.8), (3.9), отвечающие значениям $t$ из отрезков $\left[\tau_{\left(x_{0}, \varphi\right)}^{(3)}(\varepsilon), h\right],\left[\tau_{3}\left(x_{0}, \varepsilon\right), h\right]$, по аналогии с фрагментом $\Gamma_{4}(\varepsilon)$ траектории (1.3) назовем участками медленного движения. Данные участки характерны тем, что на них переменная $y$ экспоненциально мала (точнее говоря, $\left.y \leqslant \exp \left(-\varepsilon^{-3 / 4}\right)\right)$, а переменная $x$ монотонно убывает (см. рис. 5$)$. В отличие от трех предыдущих участков в качестве параметра на $\Gamma_{4}(\varepsilon), \Lambda_{4}(\varepsilon)$ оставим $t$, а вместо переменной $y$ будем рассматривать $v=\varepsilon \ln y$. В этом случае справедливо следующее утверждение.

Лемма 3.7. Для участка медленного движения $\Lambda_{4}(\varepsilon)$ имеет место равенcmso

$$
\Lambda_{4}(\varepsilon)=\left\{(t, x, y): t \in\left[\tau_{3}\left(x_{0}, \varepsilon\right), h\right], x=x\left(t, x_{0}, \varepsilon\right), y=\exp \frac{v\left(t, x_{0}, \varepsilon\right)}{\varepsilon}\right\}
$$

где функиии $x\left(t, x_{0}, \varepsilon\right), v\left(t, x_{0}, \varepsilon\right)$ при $\varepsilon \rightarrow 0$ допускают равномерные по начальному условию $x_{0} \in[a, b]$ и по $t \in\left[\tau_{3}\left(x_{0}, \varepsilon\right), h\right]$ асимптотические представления

$$
\begin{gathered}
x\left(t, x_{0}, \varepsilon\right)=\left.x(t, s)\right|_{s=x_{1}\left(x_{0}\right)}+O(\sqrt{\varepsilon}), \\
\frac{\partial x}{\partial x_{0}}\left(t, x_{0}, \varepsilon\right)=\left.\frac{f(x(t, s), 0)}{f(s, 0)}\right|_{s=x_{1}\left(x_{0}\right)} \cdot x_{1}^{\prime}\left(x_{0}\right)+O(\sqrt{\varepsilon}), \\
v\left(t, x_{0}, \varepsilon\right)=v\left(t, x_{0}\right)+O(\sqrt{\varepsilon}), \\
\frac{\partial v}{\partial x_{0}}\left(t, x_{0}, \varepsilon\right)=\left.\frac{g(x(t, s), 0)-g(s, 0)}{f(s, 0)}\right|_{s=x_{1}\left(x_{0}\right)} \cdot x_{1}^{\prime}\left(x_{0}\right)+O(\sqrt{\varepsilon}) .
\end{gathered}
$$


Здесь

$$
v\left(t, x_{0}\right)=\left.\int_{0}^{t} g(x(\tau, s), 0) d \tau\right|_{s=x_{1}\left(x_{0}\right)}=\left.\gamma_{1}\left(x(t, s), x_{1}\right)\right|_{s=x_{1}\left(x_{0}\right), x_{1}=x_{1}\left(x_{0}\right)},
$$

$\gamma_{1}\left(x, x_{1}\right), x_{1}\left(x_{0}\right)$ - функции из $(2.7),(2.10)$, а $x(t, s)$ - решение задачи Коши

$$
\dot{x}=f(x, 0),\left.\quad x\right|_{t=0}=s, \quad s>0 .
$$

ДокАЗАТЕЛЬство. Из (3.7) следует, что фигурирующие в (3.118) функции $x\left(t, x_{0}, \varepsilon\right), v\left(t, x_{0}, \varepsilon\right)$ удовлетворяют задаче Коши

$$
\begin{gathered}
\dot{x}=f\left(x, \exp \frac{v}{\varepsilon}\right), \quad \dot{v}=g\left(x, \exp \frac{v}{\varepsilon}\right), \\
\left.x\right|_{t=\tau_{3}\left(x_{0}, \varepsilon\right)}=\left.\widetilde{\widetilde{x}}\left(y, x_{0}, \varepsilon\right)\right|_{y=\exp \left(-\varepsilon^{-3 / 4}\right)},\left.\quad v\right|_{t=\tau_{3}\left(x_{0}, \varepsilon\right)}=-\varepsilon^{1 / 4} .
\end{gathered}
$$

Асимптотический анализ этой задачи проведем при априорном условии

$$
v\left(t, x_{0}, \varepsilon\right) \leqslant-\varepsilon^{1 / 4} \quad \forall t \in\left[\tau_{3}\left(x_{0}, \varepsilon\right), h\right] .
$$

Опираясь на оценку (3.125) и отбрасывая в правых частях системы (3.123) слагаемые порядка $\exp \left(-\varepsilon^{-3 / 4}\right)$, получаем более простую систему

$$
\dot{x}=f(x, 0), \quad \dot{v}=g(x, 0) .
$$

Далее, из (3.126) очевидным образом следует, что с точностью до экспоненциально малых добавок компонента $x\left(t, x_{0}, \varepsilon\right)$ определяется из задачи Коши

$$
\dot{x}=f(x, 0),\left.\quad x\right|_{t=\tau_{3}\left(x_{0}, \varepsilon\right)}=\left.\widetilde{\widetilde{x}}\left(y, x_{0}, \varepsilon\right)\right|_{y=\exp \left(-\varepsilon^{-3 / 4}\right)},
$$

а компонента $v\left(t, x_{0}, \varepsilon\right)$ задается равенством

$$
v\left(t, x_{0}, \varepsilon\right)=-\varepsilon^{1 / 4}+\int_{\tau_{3}\left(x_{0}, \varepsilon\right)}^{t} g\left(x\left(\tau, x_{0}, \varepsilon\right), 0\right) d \tau .
$$

Для анализа задачи (3.127) привлечем известные формулы (3.114), (3.115) и вытекающие из (3.101), (3.102), (3.49), (3.50) асимптотические представления

$$
\begin{aligned}
\left.\widetilde{\widetilde{x}}\left(y, x_{0}, \varepsilon\right)\right|_{y=\exp \left(-\varepsilon^{-3 / 4}\right)} & =\left.\left(x-\frac{f(x, 0)}{g(x, 0)} \varepsilon^{1 / 4}\right)\right|_{x=x_{1}\left(x_{0}\right)}+O(\sqrt{\varepsilon}), \\
\left.\frac{\partial}{\partial x_{0}} \widetilde{\widetilde{x}}\left(y, x_{0}, \varepsilon\right)\right|_{y=\exp \left(-\varepsilon^{-3 / 4}\right)} & =\frac{\partial}{\partial x_{0}}\left[\left.\left(x-\frac{f(x, 0)}{g(x, 0)} \varepsilon^{1 / 4}\right)\right|_{x=x_{1}\left(x_{0}\right)}\right]+O(\sqrt{\varepsilon}) .
\end{aligned}
$$

В результате убеждаемся, что функция $x\left(t, x_{0}, \varepsilon\right)$ при $\varepsilon \rightarrow 0$ равномерно по $x_{0} \in[a, b]$ и по $t \in\left[\tau_{3}\left(x_{0}, \varepsilon\right), h\right]$ обладает требуемой асимптотикой (3.119). Учитывая затем уже установленные равенства (3.119) вместе со свойствами (3.114), $(3.115)$ в правой части из (3.128), для компоненты $v\left(t, x_{0}, \varepsilon\right)$ получаем нужные асимптотические формулы (3.120). 
Итак, мы показали, что при условии (3.125) асимптотические представления (3.119), (3.120) действительно выполняются. Покажем теперь, что справедливо и само условие (3.125). В связи с этим остановимся на некоторых свойствах функции (3.121).

В первую очередь обратим внимание, что в силу $(2.10),(2.12)$ имеют место соотношения

$$
\begin{gathered}
\gamma_{1}\left(x, x_{1}\left(x_{0}\right)\right)<0 \quad \text { при } \quad x_{3}\left(x_{0}\right)<x<x_{1}\left(x_{0}\right), \\
\frac{\partial \gamma_{1}}{\partial x}\left(x_{1}\left(x_{0}\right), x_{1}\left(x_{0}\right)\right)>0, \quad \frac{\partial \gamma_{1}}{\partial x}\left(x_{3}\left(x_{0}\right), x_{1}\left(x_{0}\right)\right)<0,
\end{gathered}
$$

где, напомним, $x_{3}\left(x_{0}\right) \in\left(0, q_{2}\right)$ - корень уравнения $(2.16)$ при $x_{1}=x_{1}\left(x_{0}\right)$. Кроме того, согласно условию $(2.4)$ решение $\left.x(t, s)\right|_{s=x_{1}\left(x_{0}\right)}$ задачи Коши (3.122) монотонно убывает и при $t=h\left(x_{0}\right)$ (см. (3.2)) достигает значения $x_{3}\left(x_{0}\right)$.

Суммируя перечисленные факты, приходим к выводу, что $v\left(t, x_{0}\right)<0$ при

$$
0<t<h\left(x_{0}\right), \quad v\left(0, x_{0}\right)=v\left(h\left(x_{0}\right), x_{0}\right)=0,\left.\quad \frac{\partial v}{\partial t}\right|_{t=0}=g\left(x_{1}\left(x_{0}\right), 0\right)<0 .
$$

А так как $h<h\left(x_{0}\right)$ (см. (3.2)), то на интересующем нас отрезке $0 \leqslant t \leqslant h$ функция (3.121) обладает свойствами

$$
v\left(0, x_{0}\right)=0, \quad \frac{\partial v}{\partial t}\left(0, x_{0}\right)<0, \quad v\left(t, x_{0}\right)<0 \quad \forall t \in(0, h] .
$$

Остается добавить, что из (3.120), (3.124), (3.130) требуемое неравенство (3.125) вытекает очевидным образом. Лемма 3.7 доказана.

Переход от участка $\Lambda_{4}(\varepsilon)$ к $\Gamma_{4}(\varepsilon)$ в отличие от предыдущих случаев не требует каких-либо дополнительных усилий. Действительно, функции $x_{\left(x_{0}, \varphi\right)}(t, \varepsilon)$, $v_{\left(x_{0}, \varphi\right)}(t, \varepsilon)=\varepsilon \ln y_{\left(x_{0}, \varphi\right)}(t, \varepsilon)$ на отрезке $t \in\left[\tau_{\left(x_{0}, \varphi\right)}^{(3)}(\varepsilon), h\right]$ определяются из задачи Коши

$$
\begin{gathered}
\dot{x}=f\left(x, \exp \frac{v}{\varepsilon}\right), \quad \dot{v}=g\left(x, \exp \frac{v}{\varepsilon}\right)-\exp \frac{\varphi(t-h)}{\varepsilon}, \\
\left.x\right|_{t=\tau_{\left(x_{0}, \varphi\right)}^{(3)}(\varepsilon)}=\left.\widetilde{\widetilde{x}}_{x_{0}, \varphi}(y, \varepsilon)\right|_{y=\exp \left(-\varepsilon^{-3 / 4}\right)},\left.\quad v\right|_{t=\tau_{\left(x_{0}, \varphi\right)}^{(3)}(\varepsilon)}=-\varepsilon^{1 / 4},
\end{gathered}
$$

которая асимптотически близка к (3.123), (3.124). Точнее говоря, согласно (3.106), (3.116) начальные условия (3.124) и (3.132) близки с точностью до величин порядка $\exp (-M / \varepsilon), M=$ const $>0$, а фигурирующее в (3.131) дополнительное слагаемое $\exp (\varphi(t-h) / \varepsilon)$ интегрально мало (см. (3.5), (3.6)). Тем самым, для задачи (3.131), (3.132) остаются в силе все построения, проделанные при доказательстве леммы 3.7, а значит, справедливо следующее утверждение.

ЛЕмма 3.8. Участок медленного движения $\Gamma_{4}(\varepsilon)$ задается аналогичным (3.118) равенством

$$
\Gamma_{4}(\varepsilon)=\left\{(t, x, y): \tau_{\left(x_{0}, \varphi\right)}^{(3)}(\varepsilon) \leqslant t \leqslant h, x=x_{\left(x_{0}, \varphi\right)}(t, \varepsilon), y=\exp \frac{v_{\left(x_{0}, \varphi\right)}(t, \varepsilon)}{\varepsilon}\right\},
$$


причем для любого фиксированного $\delta \in(0, h)$ при $\varepsilon \rightarrow 0$ выполняются равномерные по начальным условиям $\left(x_{0}, \varphi\right) \in S$ u nо $t \in\left[\tau_{\left(x_{0}, \varphi\right)}^{(3)}(\varepsilon), h\right]$ асимптотические представления

$$
\begin{aligned}
& x_{\left(x_{0}, \varphi\right)}(t, \varepsilon)=x\left(t, x_{0}, \varepsilon\right)+ \begin{cases}O\left(\exp \left(-\frac{M}{\varepsilon}\right)\right) & \text { npu } \tau_{\left(x_{0}, \varphi\right)}^{(3)}(\varepsilon) \leqslant t \leqslant h-\delta, \\
O(\varepsilon) & n p u h-\delta \leqslant t \leqslant h,\end{cases} \\
& v_{\left(x_{0}, \varphi\right)}(t, \varepsilon)=v\left(t, x_{0}, \varepsilon\right)+ \begin{cases}O\left(\exp \left(-\frac{M}{\varepsilon}\right)\right) & n p u \tau_{\left(x_{0}, \varphi\right)}^{(3)}(\varepsilon) \leqslant t \leqslant h-\delta, \\
O(\varepsilon) & n p u h-\delta \leqslant t \leqslant h .\end{cases}
\end{aligned}
$$

Суммируя результаты лемм 3.1-3.8, мы можем утверждать, что на отрезке $t \in[0, h-\delta]$ для компонент $x_{\left(x_{0}, \varphi\right)}(t, \varepsilon), y_{\left(x_{0}, \varphi\right)}(t, \varepsilon)$ решения задачи Коши $(1.25)$, (2.26) справедливы равномерные по $\left(x_{0}, \varphi\right) \in S$ и $t$ асимптотические равенства

$$
\begin{gathered}
x_{\left(x_{0}, \varphi\right)}(t, \varepsilon)=x\left(t, x_{0}, \varepsilon\right)+O\left(\exp \left(-\frac{M}{\varepsilon}\right)\right), \\
y_{\left(x_{0}, \varphi\right)}(t, \varepsilon)=y\left(t, x_{0}, \varepsilon\right)\left(1+O\left(\exp \left(-\frac{M}{\varepsilon}\right)\right)\right), \quad M=\text { const }>0,
\end{gathered}
$$

где $x\left(t, x_{0}, \varepsilon\right), y\left(t, x_{0}, \varepsilon\right)$ - компоненты решения задачи Коши (3.7). В случае же, когда $t$ принадлежит отрезку $[h-\delta, h]$, остатки в $(3.136)$ становятся величинами порядка $\varepsilon$.

3.2. Завершение асимптотического анализа задачи Коши. Заключительный этап построения асимптотики функций $x_{\left(x_{0}, \varphi\right)}(t, \varepsilon), y_{\left(x_{0}, \varphi\right)}(t, \varepsilon)$ связан с рассмотрением промежутка времени $h \leqslant t \leqslant t_{\left(x_{0}, \varphi\right)}^{(2)}(\varepsilon)$, где, напомним, $t_{\left(x_{0}, \varphi\right)}^{(2)}(\varepsilon)$ - второй положительный корень уравнения (2.27) (существование которого еще предстоит доказать). Для удобства последующего анализа разобьем упомянутый промежуток на несколько частей.

Обратимся сначала к отрезку $h \leqslant t \leqslant h+\delta$, где постоянная $\delta>0$ подходящим образом мала. А именно, будем считать, что

$$
\delta<\frac{1}{2} \min \left\{h, \min _{a \leqslant x_{0} \leqslant b} T\left(x_{0}\right)-h\right\} .
$$

Здесь

$$
T\left(x_{0}\right)=-\int_{x_{2}\left(x_{0}\right)}^{x_{1}\left(x_{0}\right)} \frac{d s}{f(s, 0)}>h\left(x_{0}\right), \quad T\left(x_{0}^{*}\right)=T_{*},
$$

где, напомним, $x_{2}=x_{2}\left(x_{0}\right)$ - корень уравнения $(2.11)$ при $x_{1}=x_{1}\left(x_{0}\right)$, а $T_{*}-$ величина из (2.19). Кроме того, предполагаем, что

$$
\left.x(t, s)\right|_{s=x_{1}\left(x_{0}\right)} \in(a, b) \quad \forall t \in\left[T\left(x_{0}\right)-\delta, T\left(x_{0}\right)+\delta\right], \quad \forall x_{0} \in[a, b],
$$


где $\left.x(t, s)\right|_{s=x_{1}\left(x_{0}\right)}-$ функция из (3.119). Подчеркнем, что при $\delta=0$ требование (3.139) заведомо выполняется, так как в силу (3.1), (3.138) имеем

$$
\left.x(t, s)\right|_{t=T\left(x_{0}\right), s=x_{1}\left(x_{0}\right)}=x_{2}\left(x_{0}\right)=\psi\left(x_{0}\right) \in(a, b) .
$$

При $t \in[h, h+\delta]$ функции $x_{\left(x_{0}, \varphi\right)}(t, \varepsilon), v_{\left(x_{0}, \varphi\right)}(t, \varepsilon)=\varepsilon \ln y_{\left(x_{0}, \varphi\right)}(t, \varepsilon)$ определяются из аналогичной (3.131), (3.132) задачи Коши

$$
\begin{gathered}
\dot{x}=f\left(x, \exp \frac{v}{\varepsilon}\right), \quad \dot{v}=g\left(x, \exp \frac{v}{\varepsilon}\right)-y_{\left(x_{0}, \varphi\right)}(t-h, \varepsilon), \\
\left.x\right|_{t=h}=x_{\left(x_{0}, \varphi\right)}(h, \varepsilon),\left.\quad v\right|_{t=h}=v_{\left(x_{0}, \varphi\right)}(h, \varepsilon),
\end{gathered}
$$

начальные условия в которой и функция $y_{\left(x_{0}, \varphi\right)}(t-h, \varepsilon)$ уже известны. Анализ этой задачи будем проводить при аналогичном (3.125) априорном предположении

$$
v_{\left(x_{0}, \varphi\right)}(t, \varepsilon) \leqslant-M, \quad M=\text { const }>0 .
$$

Из оценки (3.141) очевидным образом следует, что фигурирующие в системе $(3.140)$ функции $f(x, \exp (v / \varepsilon)), g(x, \exp (v / \varepsilon))$ с точностью до величин порядка $\exp (-M / \varepsilon), M=$ const $>0$ близки к $f(x, 0), g(x, 0)$. А отсюда и из асимптотических равенств для $x_{\left(x_{0}, \varphi\right)}(h, \varepsilon), v_{\left(x_{0}, \varphi\right)}(h, \varepsilon)($ см. $(3.119),(3.120),(3.133)-(3.135))$ заключаем, что при $\varepsilon \rightarrow 0$ равномерно по $\left(x_{0}, \varphi\right) \in S, t \in[h, h+\delta]$ имеют место представления

$$
\begin{gathered}
x_{\left(x_{0}, \varphi\right)}(t, \varepsilon)=\left.x(t, s)\right|_{s=x_{1}\left(x_{0}\right)}+O(\sqrt{\varepsilon}) \\
v_{\left(x_{0}, \varphi\right)}(t, \varepsilon)=v\left(t, x_{0}\right)-\int_{0}^{t-h} y_{\left(x_{0}, \varphi\right)}(\tau, \varepsilon) d \tau+O(\sqrt{\varepsilon}) .
\end{gathered}
$$

Отдельного рассмотрения требует вопрос об асимптотическом поведении интегрального слагаемого из (3.142). Справедливо следующее утверждение.

Лемма 3.9. При $\varepsilon \rightarrow 0$ равномерно по $\left(x_{0}, \varphi\right) \in S, t \in\left[\tau_{\left(x_{0}, \varphi\right)}^{(3)}(\varepsilon)+h, h+\delta\right]$ выполняется асимптотическое равенство

$$
\int_{0}^{t-h} y_{\left(x_{0}, \varphi\right)}(\tau, \varepsilon) d \tau=\int_{x_{0}}^{x_{1}\left(x_{0}\right)} \frac{d s}{a_{0}(s)}+O(\sqrt{\varepsilon}) .
$$

ДокАЗАтЕльСтво. Обозначим через $I_{\left(x_{0}, \varphi\right)}^{(k)}(\varepsilon), k=1,2,3$, интегралы от функции $y_{\left(x_{0}, \varphi\right)}(t, \varepsilon)$ по отрезкам

$$
0 \leqslant t \leqslant \tau_{\left(x_{0}, \varphi\right)}^{(1)}(\varepsilon), \quad \tau_{\left(x_{0}, \varphi\right)}^{(1)}(\varepsilon) \leqslant t \leqslant \tau_{\left(x_{0}, \varphi\right)}^{(2)}(\varepsilon), \quad \tau_{\left(x_{0}, \varphi\right)}^{(2)}(\varepsilon) \leqslant t \leqslant \tau_{\left(x_{0}, \varphi\right)}^{(3)}(\varepsilon)
$$

соответственно. Для первого из них согласно формулам (3.11)-(3.16), (3.25), (3.26) имеем

$$
\begin{aligned}
I_{\left(x_{0}, \varphi\right)}^{(1)}(\varepsilon) & =\int_{1}^{\varepsilon^{-3 / 4}} y \frac{\partial}{\partial y} \theta_{x_{0}, \varphi}^{(1)}(y, \varepsilon) d y=\varepsilon \int_{1}^{\varepsilon^{-3 / 4}}\left(\frac{1}{g\left(x_{0}, y\right)}+O\left(\varepsilon^{1 / 4}\right)\right) d y \\
& =\frac{\varepsilon^{1 / 4}}{b_{0}\left(x_{0}\right)}+O(\sqrt{\varepsilon}) .
\end{aligned}
$$


Далее, соотношения (3.45)-(3.55), (3.70)-(3.73) приводят к равенствам

$$
\begin{aligned}
I_{\left(x_{0}, \varphi\right)}^{(2)}(\varepsilon) & =\frac{1}{\varepsilon} \int_{\bar{x}_{x_{0}, \varphi}(\varepsilon)}^{\overline{\bar{x}}_{x_{0}, \varphi}(\varepsilon)} u_{x_{0}, \varphi}(x, \varepsilon) \frac{d x}{f\left(x, u_{x_{0}, \varphi}(x, \varepsilon) / \varepsilon\right)} \\
& =\int_{\bar{x}_{x_{0}, \varphi}(\varepsilon)}^{\bar{x}_{x_{0}, \varphi}(\varepsilon)} \frac{1}{a_{0}(x)}\left(1+O\left(\frac{\varepsilon}{\gamma_{0}\left(x, x_{0}\right)}\right)\right) d x \\
& =\int_{x_{0}}^{x_{1}\left(x_{0}\right)} \frac{d s}{a_{0}(s)}+\left(\frac{1}{b_{0}\left(x_{1}\left(x_{0}\right)\right)}-\frac{1}{b_{0}\left(x_{0}\right)}\right) \varepsilon^{1 / 4}+O(\sqrt{\varepsilon})
\end{aligned}
$$

а из формул (3.90)-(3.96), (3.104)-(3.106) вытекает, что

$$
\begin{aligned}
I_{\left(x_{0}, \varphi\right)}^{(3)}(\varepsilon) & =\int_{\varepsilon^{-3 / 4}}^{\exp \left(-\varepsilon^{-3 / 4}\right)} y \frac{\partial}{\partial y} \theta_{x_{0}, \varphi}^{(3)}(y, \varepsilon) d y \\
& =\varepsilon \int_{\varepsilon^{-3 / 4}}^{\exp \left(-\varepsilon^{-3 / 4}\right)}\left(\frac{1}{g\left(x_{1}\left(x_{0}\right), y\right)}+O\left(\varepsilon^{1 / 4}\right)\right) d y=-\frac{\varepsilon^{1 / 4}}{b_{0}\left(x_{1}\left(x_{0}\right)\right)}+O(\sqrt{\varepsilon}) .
\end{aligned}
$$

Проделанные подсчеты показывают, что при $\varepsilon \rightarrow 0$ равномерно по начальным условиям $\left(x_{0}, \varphi\right) \in S$ и по $t \in\left[\tau_{\left(x_{0}, \varphi\right)}^{(3)}(\varepsilon)+h, h+\delta\right]$ справедливо асимптотическое представление

$$
\int_{0}^{t-h} y_{\left(x_{0}, \varphi\right)}(\tau, \varepsilon) d \tau=\int_{x_{0}}^{x_{1}\left(x_{0}\right)} \frac{d s}{a_{0}(s)}+\int_{\tau_{\left(x_{0}, \varphi\right)}^{(3)}(\varepsilon)}^{t-h} y_{\left(x_{0}, \varphi\right)}(\tau, \varepsilon) d \tau+O(\sqrt{\varepsilon}),
$$

отличающееся от (3.143) лишь одним интегральным слагаемым. Последнее же, как нетрудно увидеть, экспоненциально мало. Действительно, поскольку в силу условия $\delta<h$ (см. (3.137)) имеют место включения

$$
\left[\tau_{\left(x_{0}, \varphi\right)}^{(3)}(\varepsilon), t-h\right] \subset\left[\tau_{\left(x_{0}, \varphi\right)}^{(3)}(\varepsilon), \delta\right] \subset\left[\tau_{\left(x_{0}, \varphi\right)}^{(3)}(\varepsilon), h\right],
$$

то для $y_{\left(x_{0}, \varphi\right)}(\tau, \varepsilon)$ мы вправе воспользоваться соотношением

$$
y_{\left(x_{0}, \varphi\right)}(\tau, \varepsilon)=\exp \frac{v_{\left(x_{0}, \varphi\right)}(\tau, \varepsilon)}{\varepsilon},
$$

где $v_{\left(x_{0}, \varphi\right)}(\tau, \varepsilon)$ - компонента решения задачи Коши (3.131), (3.132). А отсюда и из $(3.120),(3.135)$ выводим оценки

$$
\begin{gathered}
\int_{\tau_{\left(x_{0}, \varphi\right)}^{(3)}(\varepsilon)}^{t-h} y_{\left(x_{0}, \varphi\right)}(\tau, \varepsilon) d \tau \leqslant \int_{\tau_{\left(x_{0}, \varphi\right)}^{(3)}(\varepsilon)}^{h} y_{\left(x_{0}, \varphi\right)}(\tau, \varepsilon) d \tau \leqslant M \exp \left(-\varepsilon^{-3 / 4}\right), \\
M=\text { const }>0 .
\end{gathered}
$$

Лемма 3.9 доказана.

Установленная лемма свидетельствует о том, что на отрезке $0 \leqslant t \leqslant h$ поведение при $\varepsilon \rightarrow 0$ функции $y_{\left(x_{0}, \varphi\right)}(t, \varepsilon)$ носит $\delta$-образный характер: согласно (3.144)-(3.147) интеграл от нее по асимптотически малому промежутку $\left[0, \tau_{\left(x_{0}, \varphi\right)}^{(3)}(\varepsilon)\right]$ при $\varepsilon \rightarrow 0$ имеет конечный предел, а интеграл по оставшейся части $\left[\tau_{\left(x_{0}, \varphi\right)}^{(3)}(\varepsilon), h\right]$ экспоненциально мал (см. (3.148)). Указанное поведение $y_{\left(x_{0}, \varphi\right)}(t, \varepsilon)$ отражается на компоненте $v_{\left(x_{0}, \varphi\right)}(t, \varepsilon)$ следующим образом: из 
(3.142), (3.143) вытекает, что при $\varepsilon \rightarrow 0$ для нее выполняется равномерное по $\left(x_{0}, \varphi\right) \in S$ и по $t \in\left[\tau_{\left(x_{0}, \varphi\right)}^{(3)}(\varepsilon)+h, h+\delta\right]$ асимптотическое равенство

$$
v_{\left(x_{0}, \varphi\right)}(t, \varepsilon)=w\left(t, x_{0}\right)+O(\sqrt{\varepsilon}),
$$

где

$$
w\left(t, x_{0}\right)=v\left(t, x_{0}\right)-\int_{x_{0}}^{x_{1}\left(x_{0}\right)} \frac{d s}{a_{0}(s)} .
$$

Завершая рассмотрение отрезка времени $h \leqslant t \leqslant h+\delta$, напомним, что асимптотические представления (3.142), (3.149) были выведены при априорном предположении (3.141). Для его проверки обратим внимание, что функция (3.150) обладает свойствами

$$
\begin{gathered}
w\left(t, x_{0}\right)<0 \quad \text { при } h \leqslant t<T\left(x_{0}\right),\left.\quad w\left(t, x_{0}\right)\right|_{t=T\left(x_{0}\right)}=0, \\
\left.\frac{\partial w}{\partial t}\left(t, x_{0}\right)\right|_{t=T\left(x_{0}\right)}=g\left(x_{2}\left(x_{0}\right), 0\right)>0
\end{gathered}
$$

где $T\left(x_{0}\right)$ - момент времени (3.138). А так как $h+\delta<\min _{a \leqslant x_{0} \leqslant b} T\left(x_{0}\right)$ (см. $(3.137))$, то имеем $w\left(t, x_{0}\right)<0$ для всех $t \in[h, h+\delta]$.

Объединяя факт отрицательности $w\left(t, x_{0}\right)$ с оценкой $v\left(h, x_{0}\right)<0$ (см. (3.130)), приходим к выводу, что требуемое неравенство (3.141) действительно выполняется при фиксированном $M$, удовлетворяющем условию

$$
0<M<\min _{a \leqslant x_{0} \leqslant b}\left[\min \left\{-v\left(h, x_{0}\right),-\max _{h \leqslant t \leqslant h+\delta} w\left(t, x_{0}\right)\right\}\right],
$$

и при всех достаточно малых $\varepsilon>0$.

Перейдем теперь к отрезку $h+\delta \leqslant t \leqslant \min \left(T\left(x_{0}\right)-\delta, 2 h+\delta\right)$, длина которого в силу (3.137) положительна. При данных значениях $t$ интересующие нас функции $x_{\left(x_{0}, \varphi\right)}(t, \varepsilon), v_{\left(x_{0}, \varphi\right)}(t, \varepsilon)$ удовлетворяют аналогичной $(3.140)$ задаче Коши

$$
\begin{gathered}
\dot{x}=f\left(x, \exp \frac{v}{\varepsilon}\right), \quad \dot{v}=g\left(x, \exp \frac{v}{\varepsilon}\right)-\exp \frac{v_{\left(x_{0}, \varphi\right)}(t-h, \varepsilon)}{\varepsilon}, \\
\left.x\right|_{t=h+\delta}=x_{\left(x_{0}, \varphi\right)}(h+\delta, \varepsilon),\left.\quad v\right|_{t=h+\delta}=v_{\left(x_{0}, \varphi\right)}(h+\delta, \varepsilon),
\end{gathered}
$$

где функция $v_{\left(x_{0}, \varphi\right)}(t-h, \varepsilon)$ и начальные условия уже известны. Анализ этой задачи проведем в предположениях

$$
v_{\left(x_{0}, \varphi\right)}(t-h, \varepsilon) \leqslant-M_{1}, \quad v_{\left(x_{0}, \varphi\right)}(t, \varepsilon) \leqslant-M_{2}, \quad M_{1}, M_{2}=\text { const }>0 .
$$

Впрочем, первое из этих условий на рассматриваемом отрезке изменения $t$ заведомо выполняется, что следует из формул (3.120), (3.135), (3.142), (3.149) и отмеченных выше свойств (3.130), (3.151) функций $v\left(t, x_{0}\right), w\left(t, x_{0}\right)$. Справедливости же второго приходится требовать a priori.

Оценки (3.154) позволяют отбросить в правых частях системы (3.152) слагаемые порядка $\exp (-M / \varepsilon), M=$ const $>0$, и перейти к системе (3.126). Далее, 
согласно равенствам (3.153) и (3.142), (3.149) эту систему следует дополнить начальными условиями

$$
\left.x\right|_{t=h+\delta}=\left.x(h+\delta, s)\right|_{s=x_{1}\left(x_{0}\right)}+O(\sqrt{\varepsilon}),\left.\quad v\right|_{t=h+\delta}=w\left(h+\delta, x_{0}\right)+O(\sqrt{\varepsilon}) .
$$

Несложный анализ получившейся задачи Коши (3.126), (3.155) приводит к равномерным по $\left(x_{0}, \varphi\right) \in S, t \in\left[h+\delta, \min \left(T\left(x_{0}\right)-\delta, 2 h+\delta\right)\right]$ асимптотическим представлениям

$$
x_{\left(x_{0}, \varphi\right)}(t, \varepsilon)=\left.x(t, s)\right|_{s=x_{1}\left(x_{0}\right)}+O(\sqrt{\varepsilon}), \quad v_{\left(x_{0}, \varphi\right)}(t, \varepsilon)=w\left(t, x_{0}\right)+O(\sqrt{\varepsilon}) .
$$

Для придания формулам (3.156) законной силы осталось убедиться в справедливости второго условия (3.154). Из второго равенства (3.156) и свойств (3.151) следует, что оно будет выполняться при

$$
0<M_{2}<\min _{a \leqslant x_{0} \leqslant b}\left(-\max _{h+\delta \leqslant t \leqslant T\left(x_{0}\right)-\delta} w\left(t, x_{0}\right)\right) .
$$

Интересно отметить, что область применимости асимптотических представлений (3.156) можно расширить с помощью метода шагов. А именно, рассматривая последовательно отрезки времени длины не более $h$ и рассуждая на каждом из них подобно описанному выше, нетрудно показать, что формулы (3.156) остаются в силе до тех пор, пока справедлива оценка $v_{\left(x_{0}, \varphi\right)}(t, \varepsilon) \leqslant-\varepsilon^{1 / 4}$, т.е. при $h+\delta \leqslant t \leqslant \tau_{\left(x_{0}, \varphi\right)}^{(4)}(\varepsilon)$. Здесь $\tau_{\left(x_{0}, \varphi\right)}^{(4)}(\varepsilon)-$ второй положительный корень первого уравнения (3.89), для которого из (3.156) вытекает равномерное по $\left(x_{0}, \varphi\right) \in S$ асимптотическое равенство

$$
\tau_{\left(x_{0}, \varphi\right)}^{(4)}(\varepsilon)=T\left(x_{0}\right)-\frac{\varepsilon^{1 / 4}}{g\left(x_{2}\left(x_{0}\right), 0\right)}+O(\sqrt{\varepsilon}) .
$$

На оставшемся промежутке $\tau_{\left(x_{0}, \varphi\right)}^{(4)}(\varepsilon) \leqslant t \leqslant t_{\left(x_{0}, \varphi\right)}^{(2)}(\varepsilon)$ снова работают развитые в предыдущем пункте геометрические методы. А именно, построение асимптотики кривой

$$
\left\{(t, x, y): \tau_{\left(x_{0}, \varphi\right)}^{(4)}(\varepsilon) \leqslant t \leqslant t_{(x, \varphi)}^{(2)}(\varepsilon), x=x_{\left(x_{0}, \varphi\right)}(t, \varepsilon), y=y_{\left(x_{0}, \varphi\right)}(t, \varepsilon)\right\}
$$

проводится по той же схеме, что и в случае участков $\Gamma_{3}(\varepsilon), \Lambda_{3}(\varepsilon)$ кривых $(3.8)$, (3.9) (см. леммы 3.5, 3.6). Выбирая в качестве параметра на кривой (3.158) переменную $y \in\left[\exp \left(-\varepsilon^{-3 / 4}\right), 1\right]$, нетрудно показать, что в данном случае переменные $t, x$ как функции от $y$ обладают при $\varepsilon \rightarrow 0$ равномерной по $\left(x_{0}, \varphi\right) \in S$, $y \in\left[\exp \left(-\varepsilon^{-3 / 4}\right), 1\right]$ асимптотикой

$$
\begin{gathered}
x=x_{\left(x_{0}, \varphi\right)}\left(\tau_{\left(x_{0}, \varphi\right)}^{(4)}(\varepsilon), \varepsilon\right) \\
+\varepsilon \int_{\exp \left(-\varepsilon^{-3 / 4}\right)}^{y} \frac{f\left(x_{2}\left(x_{0}\right), s\right)}{s g\left(x_{2}\left(x_{0}\right), s\right)} d s+O\left(\varepsilon^{5 / 4} \int_{\exp \left(-\varepsilon^{-3 / 4}\right)}^{y} \frac{s+1}{s} d s\right), \\
t=\tau_{\left(x_{0}, \varphi\right)}^{(4)}(\varepsilon)+\varepsilon \int_{\exp \left(-\varepsilon^{-3 / 4}\right)}^{y} \frac{d s}{s g\left(x_{2}\left(x_{0}\right), s\right)}+O\left(\varepsilon^{5 / 4} \ln \frac{y}{\exp \left(-\varepsilon^{-3 / 4}\right)}\right), \\
\frac{\partial t}{\partial y}=\frac{\varepsilon}{y}\left(\frac{1}{g\left(x_{2}\left(x_{0}\right), y\right)}+O\left(\varepsilon^{1 / 4}\right)\right)>0,
\end{gathered}
$$


где в силу (3.156), (3.157)

$$
x_{\left(x_{0}, \varphi\right)}\left(\tau_{\left(x_{0}, \varphi\right)}^{(4)}(\varepsilon), \varepsilon\right)=\left.\left(x-\frac{f(x, 0)}{g(x, 0)} \varepsilon^{1 / 4}\right)\right|_{x=x_{2}\left(x_{0}\right)}+O(\sqrt{\varepsilon}) .
$$

Что же касается момента времени $t=t_{\left(x_{0}, \varphi\right)}^{(2)}(\varepsilon)$, то он получается из $(3.160)$ при $y=1$ и вследствие этого допускает при $\varepsilon \rightarrow 0$ равномерную по $\left(x_{0}, \varphi\right) \in S$ асимптотику

$$
t_{\left(x_{0}, \varphi\right)}^{(2)}(\varepsilon)=T\left(x_{0}\right)+O(\sqrt{\varepsilon}) .
$$

Завершая построение асимптотики решения задачи Коши (1.25), (2.26), отметим, что в силу формул $(3.142),(3.149),(3.156)$ равномерно по $\left(x_{0}, \varphi\right) \in S$, $t \in\left[t_{\left(x_{0}, \varphi\right)}^{(2)}(\varepsilon)-\delta, t_{\left(x_{0}, \varphi\right)}^{(2)}(\varepsilon)\right]$ выполняется асимптотическое равенство

$$
y_{\left(x_{0}, \varphi\right)}(t-h, \varepsilon)=O\left(\exp \left(-\frac{M}{\varepsilon}\right)\right), \quad M=\text { const }>0 .
$$

А это значит, что кривая

$$
\left\{(t, x, y): t_{\left(x_{0}, \varphi\right)}^{(2)}(\varepsilon)-\delta \leqslant t \leqslant t_{\left(x_{0}, \varphi\right)}^{(2)}(\varepsilon), x=x_{\left(x_{0}, \varphi\right)}(t, \varepsilon), y=y_{\left(x_{0}, \varphi\right)}(t, \varepsilon)\right\}
$$

аппроксимируется с экспоненциальной точностью соответствующей фазовой кривой системы из (3.7). Точнее говоря, при $t \in\left[t_{\left(x_{0}, \varphi\right)}^{(2)}(\varepsilon)-\delta, t_{\left(x_{0}, \varphi\right)}^{(2)}(\varepsilon)\right]$ справедливы аналоги формул (3.136), в которых функции $x\left(t, x_{0}, \varepsilon\right), y\left(t, x_{0}, \varepsilon\right)$ заменяются компонентами решения задачи Коши

$$
\begin{gathered}
\dot{x}=f(x, y), \quad \varepsilon \dot{y}=g(x, y) y \\
\left.x\right|_{t=t_{\left(x_{0}, \varphi\right)}^{(2)}(\varepsilon)}=x_{\left(x_{0}, \varphi\right)}\left(t_{\left(x_{0}, \varphi\right)}^{(2)}(\varepsilon), \varepsilon\right),\left.\quad y\right|_{t=t_{\left(x_{0}, \varphi\right)}^{(2)}(\varepsilon)}=1 .
\end{gathered}
$$

\section{§ 4. Существование и устойчивость релаксационного цикла}

4.1. Доказательство существования цикла. Проделанный асимптотический анализ свидетельствует о том, что оператор (2.28) корректно определен на множестве (2.25). Убедимся теперь, что при подходящем выборе констант $m_{1}, m_{2}$ в $(2.25)$ имеет место нужное свойство $\Pi_{\varepsilon}(S) \subset S$.

Заметим в первую очередь, что в силу формул (3.159), (3.162), (3.163) справедливо равномерное по $\left(x_{0}, \varphi\right) \in S$ асимптотическое представление

$$
x_{\left(x_{0}, \varphi\right)}\left(t_{\left(x_{0}, \varphi\right)}^{(2)}(\varepsilon), \varepsilon\right)=\psi\left(x_{0}\right)+O(\sqrt{\varepsilon}), \quad \varepsilon \rightarrow 0
$$

где $\psi\left(x_{0}\right)$ - отображение (2.14). А отсюда и из (3.1) заключаем, что

$$
x_{\left(x_{0}, \varphi\right)}\left(t_{\left(x_{0}, \varphi\right)}^{(2)}(\varepsilon), \varepsilon\right) \in(a, b) \quad \forall\left(x_{0}, \varphi\right) \in S .
$$

На следующем этапе покажем, что при соответствующем выборе постоянных $m_{1}, m_{2}>0$ и $\forall\left(x_{0}, \varphi\right) \in S$ на промежутке $t \in[-h, 0)$ для функции $v_{\left(x_{0}, \varphi\right)}(t, \varepsilon)=$ $\varepsilon \ln y_{\left(x_{0}, \varphi\right)}(t, \varepsilon)$ выполняются оценки

$$
m_{2} t<v_{\left(x_{0}, \varphi\right)}\left(t+t_{\left(x_{0}, \varphi\right)}^{(2)}(\varepsilon), \varepsilon\right)<m_{1} t .
$$


Попытаемся сначала оценить сверху и снизу функцию

$$
\begin{aligned}
\dot{v}_{\left(x_{0}, \varphi\right)}(t+ & \left.t_{\left(x_{0}, \varphi\right)}^{(2)}(\varepsilon), \varepsilon\right)=g\left(x_{\left(x_{0}, \varphi\right)}\left(t+t_{\left(x_{0}, \varphi\right)}^{(2)}(\varepsilon), \varepsilon\right), y_{\left(x_{0}, \varphi\right)}\left(t+t_{\left(x_{0}, \varphi\right)}^{(2)}(\varepsilon), \varepsilon\right)\right) \\
& -y_{\left(x_{0}, \varphi\right)}\left(t+t_{\left(x_{0}, \varphi\right)}^{(2)}(\varepsilon)-h, \varepsilon\right)
\end{aligned}
$$

на промежутке времени $t \in\left[T\left(x_{0}\right)-\delta-t_{\left(x_{0}, \varphi\right)}^{(2)}(\varepsilon), 0\right)$. Поскольку в этом случае справедливо включение $t+t_{\left(x_{0}, \varphi\right)}^{(2)}(\varepsilon) \in\left[T\left(x_{0}\right)-\delta, T\left(x_{0}\right)+\delta\right]$, то в силу $(3.139)$, (3.156), (3.159), (3.162) при рассматриваемых $t$ имеем

$$
a \leqslant x_{\left(x_{0}, \varphi\right)}\left(t+t_{\left(x_{0}, \varphi\right)}^{(2)}(\varepsilon), \varepsilon\right) \leqslant b .
$$

Что же касается функции $y_{\left(x_{0}, \varphi\right)}\left(t+t_{\left(x_{0}, \varphi\right)}^{(2)}(\varepsilon)-h, \varepsilon\right)$, то она при указанных значениях $t$ является величиной порядка $\exp (-M / \varepsilon), M=$ const $>0$. Действительно, так как в силу (3.137)

$$
t+t_{\left(x_{0}, \varphi\right)}^{(2)}(\varepsilon)-h \in\left[T\left(x_{0}\right)-\delta-h, T\left(x_{0}\right)+\delta-h\right] \subset\left[\delta, T\left(x_{0}\right)-\delta\right],
$$

то согласно формулам (3.135), (3.142), (3.149), (3.156) здесь справедливы оценки

$$
\begin{gathered}
v_{\left(x_{0}, \varphi\right)}\left(t+t_{\left(x_{0}, \varphi\right)}^{(2)}(\varepsilon)-h, \varepsilon\right) \leqslant-M, \\
y_{\left(x_{0}, \varphi\right)}\left(t+t_{\left(x_{0}, \varphi\right)}^{(2)}(\varepsilon)-h, \varepsilon\right)=\exp \frac{v_{\left(x_{0}, \varphi\right)}\left(t+t_{\left(x_{0}, \varphi\right)}^{(2)}(\varepsilon)-h, \varepsilon\right)}{\varepsilon} \leqslant \exp \left(-\frac{M}{\varepsilon}\right), \\
M=\text { const }>0 .
\end{gathered}
$$

Объединяя соотношения (4.4)-(4.6), приходим к выводу, что на промежутке времени $T\left(x_{0}\right)-\delta-t_{\left(x_{0}, \varphi\right)}^{(2)}(\varepsilon) \leqslant t<0$ имеют место неравенства

$$
m_{1}<\dot{v}_{\left(x_{0}, \varphi\right)}\left(t+t_{\left(x_{0}, \varphi\right)}^{(2)}(\varepsilon), \varepsilon\right)<m_{2}
$$

с постоянными $m_{1}, m_{2}$ :

$$
m_{2}>\max _{\substack{a \leqslant x \leqslant b \\ 0 \leqslant y \leqslant 1}} g(x, y), \quad 0<m_{1}<\min _{\substack{a \leqslant x \leqslant b \\ 0 \leqslant y \leqslant 1}} g(x, y)
$$

(подчеркнем, что в силу первого свойства (3.3) фигурирующие в (4.8) максимум и минимум положительны). Интегрируя, далее, получившиеся неравенства (4.7) по отрезку $[t, 0]$, убеждаемся в справедливости оценок (4.3) на промежутке $t \in\left[T\left(x_{0}\right)-\delta-t_{\left(x_{0}, \varphi\right)}^{(2)}(\varepsilon), 0\right)$.

Для распространения оценок (4.3) на оставшийся отрезок

$$
-h \leqslant t \leqslant T\left(x_{0}\right)-\delta-t_{\left(x_{0}, \varphi\right)}^{(2)}(\varepsilon)
$$

уточним выбор постоянных $m_{1}, m_{2}$. С этой целью введем в рассмотрение функции

$$
\begin{gathered}
\bar{v}\left(t, x_{0}\right)= \begin{cases}v\left(t+T\left(x_{0}\right), x_{0}\right) & \text { при } t \in\left[-T\left(x_{0}\right),-T\left(x_{0}\right)+h\right], \\
w\left(t+T\left(x_{0}\right), x_{0}\right) & \text { при } t \in\left(-T\left(x_{0}\right)+h, 0\right],\end{cases} \\
\underline{v}\left(t, x_{0}\right)= \begin{cases}v\left(t+T\left(x_{0}\right), x_{0}\right) & \text { при } t \in\left[-T\left(x_{0}\right),-T\left(x_{0}\right)+h\right), \\
w\left(t+T\left(x_{0}\right), x_{0}\right) & \text { при } t \in\left[-T\left(x_{0}\right)+h, 0\right],\end{cases}
\end{gathered}
$$


где, напомним, $v\left(t, x_{0}\right), w\left(t, x_{0}\right)$ - функции $(3.121),(3.150)$, и предположим, что

$$
m_{2} t<\underline{v}\left(t, x_{0}\right) \leqslant \bar{v}\left(t, x_{0}\right)<m_{1} t \quad \forall t \in[-h,-\delta], \quad \forall x_{0} \in[a, b] .
$$

Отметим, что поскольку в силу (3.130), (3.151) функции (4.9), (4.10) строго отрицательны при $-h \leqslant t \leqslant-\delta$, то требование (4.11) будет заведомо выполняться при подходящем увеличении $m_{2}$ и уменьшении $m_{1}$.

Указанный выбор $m_{1}, m_{2}$ приводит к желаемой цели. Действительно, из (4.11) и асимптотических представлений (3.135), (3.142), (3.149), (3.156) интересующие нас оценки (4.3) при $t \in\left[-h, T\left(x_{0}\right)-\delta-t_{\left(x_{0}, \varphi\right)}^{(2)}(\varepsilon)\right]$ вытекают автоматически.

Подведем итог. Полученные выше соотношения (4.2), (4.3), собственно говоря, и означают, что справедливо требуемое свойство $\Pi_{\varepsilon}(S) \subset S$. Учитывая это обстоятельство и опираясь на принцип Шаудера, заключаем, что оператор $\Pi_{\varepsilon}$, очевидным образом являющийся компактным, имеет в множестве $S$ по крайней мере одну неподвижную точку

$$
\left(x_{0}^{*}(\varepsilon), \varphi_{*}(t, \varepsilon)\right), \quad-h \leqslant t \leqslant 0 .
$$

Ясно также, что решение

$$
\left(x_{*}(t, \varepsilon), y_{*}(t, \varepsilon)\right)
$$

системы $(1.25)$ с начальными условиями $\left.x\right|_{t=0}=x_{0}^{*}(\varepsilon), y=\exp \left(\varphi_{*}(t, \varepsilon) / \varepsilon\right)$, $-h \leqslant t \leqslant 0$, оказывается периодическим с периодом

$$
T_{*}(\varepsilon)=\left.t_{\left(x_{0}, \varphi\right)}^{(2)}(\varepsilon)\right|_{x_{0}=x_{0}^{*}(\varepsilon), \varphi=\varphi_{*}} .
$$

Убедимся теперь, что периодическое решение (4.13) обладает требуемыми в теореме 2.1 асимптотическими свойствами (2.21)-(2.24). В связи с этим обратим внимание, что в силу (4.1) компонента $x_{0}^{*}(\varepsilon)$ неподвижной точки $(4.12)$ удовлетворяет уравнению

$$
x_{0}=\psi\left(x_{0}\right)+O(\sqrt{\varepsilon})
$$

и, следовательно, допускает асимптотику

$$
x_{0}^{*}(\varepsilon)=x_{0}^{*}+O(\sqrt{\varepsilon}), \quad \varepsilon \rightarrow 0,
$$

где, напомним, $x_{0}^{*}$ - неподвижная точка отображения (2.14). А отсюда и из (3.163), (4.14) очевидным образом имеем

$$
T_{*}(\varepsilon)=T_{*}+O(\sqrt{\varepsilon}), \quad \varepsilon \rightarrow 0,
$$

т.е. выполняется предельное равенство (2.21).

Проверка справедливости второго равенства (2.22) и соотношений (2.23), (2.24) не вызывает затруднений, поскольку они непосредственно следуют из уже установленных формул $(4.15),(4.16)$ и соответствующих асимптотических представлений для $x_{\left(x_{0}, \varphi\right)}(t, \varepsilon), y_{\left(x_{0}, \varphi\right)}(t, \varepsilon)$ (см. (3.70)-(3.73), (3.133)-(3.135), 
(3.142), (3.149), (3.156)). В некоторых пояснениях нуждается лишь первое равенство (2.22).

Для его обоснования заметим, что в силу равенств (3.117), (4.15) справедливо асимптотическое представление

$$
\left.t_{*}(\varepsilon) \stackrel{\text { def }}{=} t_{\left(x_{0}, \varphi\right)}^{(1)}(\varepsilon)\right|_{x_{0}=x_{0}^{*}(\varepsilon), \varphi=\varphi_{*}}=\left(\frac{1}{b_{0}\left(x_{0}^{*}\right)}-\frac{1}{b_{0}\left(x_{1}^{*}\right)}\right) \varepsilon \ln \frac{1}{\varepsilon}+O(\varepsilon), \quad \varepsilon \rightarrow 0,
$$

где, напомним, $x_{1}^{*}-$ решение уравнения $(2.8)$ при $x_{0}=x_{0}^{*}$. Далее, из формул (3.144)-(3.146) вытекает, что равномерно по $\left(x_{0}, \varphi\right) \in S$

$$
\int_{0}^{t_{\left(x_{0}, \varphi\right)}^{(1)}(\varepsilon)} y_{\left(x_{0}, \varphi\right)}(t, \varepsilon) d t=\int_{x_{0}}^{x_{1}\left(x_{0}\right)} \frac{d s}{a_{0}(s)}+O(\sqrt{\varepsilon}), \quad \varepsilon \rightarrow 0 .
$$

Тем самым, для получения первого предельного соотношения (2.22) остается положить в (4.18) $x_{0}=x_{0}^{*}(\varepsilon), \varphi=\varphi_{*}$ и учесть формулы (4.15), (4.17).

Итак, мы установили факт существования у системы (1.25) неклассического релаксационного цикла (4.13), обладающего нужными свойствами (2.21)-(2.24). Вопрос же об устойчивости этого цикла требует отдельного рассмотрения. Соответствующий анализ приводится ниже.

4.2. Исследование устойчивости. Как известно, проблема устойчивости цикла (4.13) сводится к анализу расположения мультипликаторов отвечающей ему системы в вариациях. Для ее вывода подставим в систему (1.25) соотношения $x=x_{*}(t, \varepsilon)+z_{1}, y=y_{*}(t, \varepsilon)+z_{2}$ и отбросим нелинейные по $z_{1}, z_{2}$ слагаемые. В результате для $z_{1}, z_{2}$ получается некоторая линейная система с $T_{*}(\varepsilon)$-периодическими коэффициентами, которая после замены

$$
\frac{\varepsilon z_{2}}{y_{*}(t, \varepsilon)} \rightarrow z_{2}
$$

преобразуется к виду

$$
\dot{z}=A(t, \varepsilon) z+B(t, \varepsilon) z(t-h),
$$

где $z=\operatorname{colon}\left(z_{1}, z_{2}\right)$,

$$
\begin{aligned}
& A(t, \varepsilon)=\left(\begin{array}{cc}
a_{11}(t, \varepsilon) & a_{12}(t, \varepsilon) \\
a_{21}(t, \varepsilon) & a_{22}(t, \varepsilon)
\end{array}\right), \quad B(t, \varepsilon)=\left(\begin{array}{cc}
0 & 0 \\
0 & -\frac{y_{*}(t-h, \varepsilon)}{\varepsilon}
\end{array}\right), \\
& a_{11}(t, \varepsilon)=f_{x}^{\prime}\left(x_{*}(t, \varepsilon), y_{*}(t, \varepsilon)\right), \quad a_{12}(t, \varepsilon)=\frac{1}{\varepsilon} y_{*}(t, \varepsilon) f_{y}^{\prime}\left(x_{*}(t, \varepsilon), y_{*}(t, \varepsilon)\right), \\
& a_{21}(t, \varepsilon)=g_{x}^{\prime}\left(x_{*}(t, \varepsilon), y_{*}(t, \varepsilon)\right), \quad a_{22}(t, \varepsilon)=\frac{1}{\varepsilon} y_{*}(t, \varepsilon) g_{y}^{\prime}\left(x_{*}(t, \varepsilon), y_{*}(t, \varepsilon)\right) \text {. }
\end{aligned}
$$

Во избежание недоразумений поясним смысл термина "мультипликатор" применительно к системе (4.20). В связи с этим рассмотрим банахово пространство $E=\mathbb{C} \times C[-h-\delta,-\delta]$ (над полем комплексных чисел), где $\delta>0$, фиксировано 
и удовлетворяет требованиям (3.137), (3.139). Точнее говоря, будем считать, что элементами пространства $E$ являются вектор-функции

$$
z_{0}(t)=\operatorname{colon}\left(z_{1}^{0}(t), z_{2}^{0}(t)\right), \quad z_{1}^{0}(t) \equiv z_{1}^{0}, \quad z_{1}^{0} \in \mathbb{C}, \quad z_{2}^{0}(t) \in C[-h-\delta,-\delta],
$$

а норму в $E$ зададим равенством

$$
\left\|z_{0}\right\|_{E}=\left|z_{1}^{0}\right|+\max _{-h-\delta \leqslant t \leqslant-\delta}\left|z_{2}^{0}(t)\right| .
$$

Далее, оператором монодромии системы (4.20) назовем ограниченный линейный оператор $U(\varepsilon): E \rightarrow E$, действующий на произвольную функцию $z_{0}(t) \in E$ по правилу

$$
U(\varepsilon) z_{0}=\operatorname{colon}\left(z_{1}\left(T_{*}(\varepsilon)-\delta, \varepsilon\right), z_{2}\left(t+T_{*}(\varepsilon), \varepsilon\right)\right), \quad-h-\delta \leqslant t \leqslant-\delta,
$$

где $z(t, \varepsilon)=\operatorname{colon}\left(z_{1}(t, \varepsilon), z_{2}(t, \varepsilon)\right)$ - решение системы (4.20) на отрезке времени $-\delta \leqslant t \leqslant T_{*}(\varepsilon)-\delta$ с начальной функцией $z_{0}(t),-h-\delta \leqslant t \leqslant-\delta$. Отметим, что спектр этого оператора дискретен, поскольку в силу неравенства $T_{*}(\varepsilon)>h$ (см. (4.16), (2.17), (2.19)) он является компактным. Что же касается мультипликаторов системы (4.20), то таковыми по аналогии со случаем обыкновенных дифференциальных уравнений будем называть собственные значения $\nu_{k}(\varepsilon), k \in \mathbb{N}$, оператора (4.24), занумерованные в порядке убывания модулей.

Наша ближайшая задача заключается в асимптотическом вычислении мультипликаторов $\nu_{k}(\varepsilon), k \geqslant 1$. Соответствующий анализ разобьем на два этапа. На первом этапе мы покажем, что оператор (4.24) экспоненциально близок к некоторому двумерному линейному оператору, а на втором - проанализируем спектр упомянутого оператора. В результате будет установлено, что

$$
\nu_{1}(\varepsilon) \equiv 1, \quad \nu_{2}(\varepsilon)=\psi^{\prime}\left(x_{0}^{*}\right)+O(\sqrt{\varepsilon}), \quad \varepsilon \rightarrow 0,
$$

а остальные мультипликаторы при всех достаточно малых $\varepsilon>0$ удовлетворяют оценке

$$
\sup _{k \geqslant 3}\left|\nu_{k}(\varepsilon)\right| \leqslant M_{1} \exp \left(-\frac{M_{2}}{\varepsilon}\right)
$$

с некоторыми универсальными постоянными $M_{1}, M_{2}>0$.

Первый этап описанного плана действий оформим в виде соответствующей леммы. Для этого нам потребуется подпространство $E_{0} \subset E$, задающееся равенством

$$
E_{0}=\left\{z_{0}(t) \in E: z_{0}(t)=\operatorname{colon}\left(0, z_{2}^{0}(t)\right), z_{2}^{0}(-\delta)=0\right\} .
$$

Договоримся также одним и тем же символом $\|*\|$ обозначать евклидову векторную норму и индуцированную ею матричную норму (из контекста всегда будет ясно какая именно норма используется).

Лемма 4.1. Найдутся такое достаточно малое $\varepsilon_{0}>0$ и такие универсальные константы $M_{1}, M_{2}>0$, что при всех $0<\varepsilon \leqslant \varepsilon_{0} u \forall z_{0}(t) \in E_{0}$ 
решение $z(t, \varepsilon)$ системы (4.20) с начальной функиией $z_{0}(t),-h-\delta \leqslant t \leqslant-\delta$ удовлетворяет оченке

$$
\max _{-\delta \leqslant t \leqslant T_{*}(\varepsilon)-\delta}\|z(t, \varepsilon)\| \leqslant M_{1} \exp \left(-\frac{M_{2}}{\varepsilon}\right)\left\|z_{0}\right\|_{E} .
$$

ДоказАтельство. Рассмотрим сначала отрезок $-\delta \leqslant t \leqslant 0$, на котором интересующее нас решение $z(t, \varepsilon)$ в силу $(4.20),(4.27)$ определяется из задачи Коши

$$
\dot{z}=A(t, \varepsilon) z+B(t, \varepsilon) z_{0}(t-h),\left.\quad z\right|_{t=-\delta}=0 .
$$

Для анализа этой задачи нам потребуются некоторые свойства матриц (4.21), а также матрицы Коши $K(t, \tau, \varepsilon), K(\tau, \tau, \varepsilon)=I$ системы

$$
\dot{z}=A(t, \varepsilon) z
$$

(здесь и ниже через $I$ обозначается единичная матрица или единичный оператор).

В случае матрицы $B(t, \varepsilon)$ ситуация достаточно проста: из неравенства $(2.24)$ очевидным образом имеем

$$
\max _{-\delta \leqslant t \leqslant h-\delta}\|B(t, \varepsilon)\| \leqslant M_{1} \exp \left(-\frac{M_{2}}{\varepsilon}\right), \quad M_{1}, M_{2}=\text { const }>0 .
$$

В случае же матриц $A(t, \varepsilon), K(t, \tau, \varepsilon)$ необходима некоторая дополнительная информация о функции $y_{*}(t, \varepsilon)$, а именно вытекающая из $(3.156),(3.159)-(3.162)$ оценка

$$
\begin{aligned}
\int_{-\delta}^{0} y_{*}(t, \varepsilon) d t & =\int_{T_{*}(\varepsilon)-\delta}^{T_{*}(\varepsilon)} y_{*}(t, \varepsilon) d t=\left.\int_{t_{\left(x_{0}, \varphi\right)}^{(2)}(\varepsilon)-\delta}^{t_{\left(x_{0}, \varphi\right)}^{(2)}(\varepsilon)} y_{\left(x_{0}, \varphi\right)}(t, \varepsilon) d t\right|_{x_{0}=x_{0}^{*}(\varepsilon), \varphi=\varphi_{*}} \\
& =\varepsilon \int_{0}^{1} \frac{d y}{g\left(x_{0}^{*}, y\right)}+O\left(\varepsilon^{5 / 4}\right) \leqslant M \varepsilon, \quad M=\mathrm{const}>0
\end{aligned}
$$

Учитывая свойство (4.32) в (4.22), последовательно выводим

$$
\begin{gathered}
\int_{-\delta}^{0}\|A(t, \varepsilon)\| d t \leqslant M_{1}, \max _{-\delta \leqslant \tau \leqslant t \leqslant 0}\|K(t, \tau, \varepsilon)\| \leqslant M_{2}, \\
M_{1}, M_{2}=\text { const }>0 .
\end{gathered}
$$

Неравенства (4.31), (4.33) позволяют без труда получить интересующую нас оценку на функцию $z(t, \varepsilon)$ при $-\delta \leqslant t \leqslant 0$. Действительно, учитывая их в явной формуле

$$
z(t, \varepsilon)=\int_{-\delta}^{t} K(t, \tau, \varepsilon) B(\tau, \varepsilon) z_{0}(\tau-h) d \tau,
$$

приходим к выводу, что на отрезке $t \in[-\delta, 0]$

$$
\max _{t}\|z(t, \varepsilon)\| \leqslant M_{1} \exp \left(-\frac{M_{2}}{\varepsilon}\right)\left\|z_{0}\right\|_{E} .
$$


Дальнейший способ действий состоит в последовательном рассмотрении различных промежутков изменения $t$, на которых сначала устанавливаются некоторые свойства матрицы Коши $K(t, \tau, \varepsilon)$ системы (4.30), а затем посредством аналогичных (4.34) формул обосновывается требуемое неравенство (4.35).

Очередной шаг связан с отрезком $t \in\left[0, \tau_{*}^{(1)}(\varepsilon)\right]$, где $\tau_{*}^{(1)}(\varepsilon)$ - момент времени $(3.44)$, вычисленный на компонентах $x_{0}=x_{0}^{*}(\varepsilon), \varphi=\varphi_{*}(t, \varepsilon)$ неподвижной точки (4.12). При указанных $t$ выполним в системе (4.30) замену времени

$$
t=\left.\theta_{x_{0}, \varphi}^{(1)}(y, \varepsilon)\right|_{x_{0}=x_{0}^{*}(\varepsilon), \varphi=\varphi_{*}}, \quad y \in\left[1, \varepsilon^{-3 / 4}\right]
$$

и учтем в ее коэффициентах (4.22) соотношения $(3.25),(3.26)$ (при $x_{0}=x_{0}^{*}(\varepsilon)$, $\left.\varphi=\varphi_{*}(t, \varepsilon)\right),(3.12),(3.14),(3.16)$. В результате интересующая нас система примет вид

$$
\frac{d z}{d y}=A(y, \varepsilon) z, \quad A(y, \varepsilon)=\left(\begin{array}{ll}
a_{11}(y, \varepsilon) & a_{12}(y, \varepsilon) \\
a_{21}(y, \varepsilon) & a_{22}(y, \varepsilon)
\end{array}\right)
$$

где равномерно по $y \in\left[1, \varepsilon^{-3 / 4}\right]$

$$
\begin{gathered}
a_{11}(y, \varepsilon)=O(\varepsilon), \quad\left|a_{12}(y, \varepsilon)\right| \leqslant M_{1}, \quad\left|a_{21}(y, \varepsilon)\right| \leqslant M_{2} \frac{\varepsilon}{y}, \\
\left|a_{22}(y, \varepsilon)\right| \leqslant \frac{M_{3}}{y^{2}}, \quad M_{j}=\text { const }>0, \quad j=1,2,3 .
\end{gathered}
$$

Положим в $(4.36) A(y, \varepsilon)=A_{0}(y, \varepsilon)+A_{1}(y, \varepsilon)$, где

$$
A_{0}(y, \varepsilon)=\left(\begin{array}{cc}
a_{11}(y, \varepsilon) & a_{12}(y, \varepsilon) \\
0 & a_{22}(y, \varepsilon)
\end{array}\right), \quad A_{1}(y, \varepsilon)=\left(\begin{array}{cc}
0 & 0 \\
a_{21}(y, \varepsilon) & 0
\end{array}\right) .
$$

Заметим далее, что матрица Коши $K_{0}(y, s, \varepsilon), K_{0}(s, s, \varepsilon)=I$ системы уравнений $d z / d y=A_{0}(y, \varepsilon) z$ выписывается в явном виде и в силу свойств (4.37) допускает оценку

$$
\max _{1 \leqslant s \leqslant y \leqslant \varepsilon^{-3 / 4}}\left\|K_{0}(y, s, \varepsilon)\right\| \leqslant M \varepsilon^{-3 / 4}, \quad M=\text { const }>0 .
$$

Что же касается матрицы Коши $K(y, s, \varepsilon)$ системы (4.36), то она удовлетворяет интегральному уравнению

$$
K(y, s, \varepsilon)=K_{0}(y, s, \varepsilon)+\int_{s}^{y} K_{0}(y, \sigma, \varepsilon) A_{1}(\sigma, \varepsilon) K(\sigma, s, \varepsilon) d \sigma,
$$

из которого в силу $(4.37),(4.38)$ имеем

$$
\|K(y, s, \varepsilon)\| \leqslant M_{1} \varepsilon^{-3 / 4}+\varepsilon^{1 / 4} M_{2} \int_{s}^{y} \frac{1}{\sigma}\|K(\sigma, s, \varepsilon)\| d \sigma, \quad M_{1}, M_{2}=\text { const }>0 .
$$

А отсюда и из леммы Гронуолла-Беллмана заключаем, что

$$
\max _{1 \leqslant s \leqslant y \leqslant \varepsilon^{-3 / 4}}\|K(y, s, \varepsilon)\| \leqslant M \varepsilon^{-3 / 4}, \quad M=\text { const }>0 .
$$


Установленная оценка на $K(y, s, \varepsilon)$ (а значит, и на матрицу $K(t, \tau, \varepsilon)$ при $\left.0 \leqslant \tau \leqslant t \leqslant \tau_{*}^{(1)}(\varepsilon)\right)$ позволяет распространить неравенство (4.35) на отрезок $0 \leqslant t \leqslant \tau_{*}^{(1)}(\varepsilon)$. Действительно, упомянутое неравенство очевидным образом вытекает из (4.31), (4.40) и из формулы

$$
z(t, \varepsilon)=K(t, 0, \varepsilon) z(0, \varepsilon)+\int_{0}^{t} K(t, \tau, \varepsilon) B(\tau, \varepsilon) z_{0}(\tau-h) d \tau
$$

Следующий шаг связан с отрезком $\tau_{*}^{(1)}(\varepsilon) \leqslant t \leqslant \tau_{*}^{(2)}(\varepsilon)$, где $\tau_{*}^{(2)}(\varepsilon)$ - момент времени (3.88) при $x_{0}=x_{0}^{*}(\varepsilon), \varphi=\varphi_{*}(t, \varepsilon)$. В этом случае сделаем в $(4.30)$ замены

$$
t=\left.\theta_{x_{0}, \varphi}^{(2)}(x, \varepsilon)\right|_{x=x_{0}^{*}(\varepsilon), \varphi=\varphi_{*}}, \quad \bar{x}_{*}(\varepsilon) \leqslant x \leqslant \overline{\bar{x}}_{*}(\varepsilon), \quad \varepsilon z_{1} \rightarrow z_{1},
$$

где (см. (3.73), (3.47), (3.49), (4.15))

$$
\begin{aligned}
& \bar{x}_{*}(\varepsilon)=\left.\bar{x}_{x_{0}, \varphi}(\varepsilon)\right|_{x_{0}=x_{0}^{*}(\varepsilon), \varphi=\varphi_{*}}=x_{0}^{*}+\frac{a_{0}\left(x_{0}^{*}\right)}{b_{0}\left(x_{0}^{*}\right)} \varepsilon^{1 / 4}+O(\sqrt{\varepsilon})>x_{0}^{*}, \\
& \overline{\bar{x}}_{*}(\varepsilon)=\left.\overline{\bar{x}}_{x_{0}, \varphi}(\varepsilon)\right|_{x_{0}=x_{0}^{*}(\varepsilon), \varphi=\varphi_{*}}=x_{1}^{*}+\frac{a_{0}\left(x_{1}^{*}\right)}{b_{0}\left(x_{1}^{*}\right)} \varepsilon^{1 / 4}+O(\sqrt{\varepsilon})<x_{1}^{*},
\end{aligned}
$$

и учтем формулы (3.72) (при $\left.x_{0}=x_{0}^{*}(\varepsilon), \varphi=\varphi_{*}(t, \varepsilon)\right),(3.51),(3.52),(3.54)$. Указанная последовательность действий приводит систему (4.30) к виду

$$
\frac{d z}{d x}=A(x, \varepsilon) z, \quad A(x, \varepsilon)=\left(\begin{array}{ll}
a_{11}(x, \varepsilon) & a_{12}(x, \varepsilon) \\
a_{21}(x, \varepsilon) & a_{22}(x, \varepsilon)
\end{array}\right),
$$

где для коэффициентов $a_{j k}(x, \varepsilon), j, k=1,2$, при $\varepsilon \rightarrow 0$ справедливы равномерные по $x \in\left[\bar{x}_{*}(\varepsilon), \overline{\bar{x}}_{*}(\varepsilon)\right]$ асимптотические представления

$$
\begin{gathered}
a_{11}(x, \varepsilon)=\frac{a_{0}^{\prime}(x)}{a_{0}(x)}+O\left(\varepsilon^{1 / 4}\right), \quad a_{12}(x, \varepsilon)=1+O\left(\varepsilon^{1 / 4}\right), \\
a_{21}(x, \varepsilon)=\frac{b_{0}^{\prime}(x)}{a_{0}(x) \gamma_{0}\left(x, x_{0}^{*}\right)}+O\left(\frac{\sqrt{\varepsilon}}{\gamma_{0}^{2}\left(x, x_{0}^{*}\right)}\right), \quad a_{22}(x, \varepsilon)=O(\sqrt{\varepsilon}) .
\end{gathered}
$$

Обозначим через $K(x, s)$ матрицу Коши системы уравнений $d z / d x=A_{0}(x) z$, где $A_{0}(x)=A(x, 0)$. Заметим далее, что $K(x, s)$ задается посредством равенств

$$
K(x, s)=K(x) K^{-1}(s), \quad K(x)=\left(\begin{array}{ll}
\mathbb{k}_{11}(x) & \mathbb{k}_{12}(x) \\
\mathbb{k}_{21}(x) & \mathbb{k}_{22}(x)
\end{array}\right),
$$

где

$$
\begin{gathered}
\mathbb{k}_{11}(x)=a_{0}(x) \gamma_{0}\left(x, x_{0}^{*}\right), \quad \mathbb{k}_{21}(x)=b_{0}(x), \\
\mathbb{k}_{12}(x)=a_{0}(x) \gamma_{0}\left(x, x_{0}^{*}\right) \int_{\widetilde{x}}^{x} \frac{d \sigma}{a_{0}(\sigma) \gamma_{0}^{2}\left(\sigma, x_{0}^{*}\right)}, \\
\mathbb{k}_{22}(x)=\frac{1}{\gamma_{0}\left(x, x_{0}^{*}\right)}+b_{0}(x) \int_{\widetilde{x}}^{x} \frac{d \sigma}{a_{0}(\sigma) \gamma_{0}^{2}\left(\sigma, x_{0}^{*}\right)}, \quad \widetilde{x}=\operatorname{const} \in\left(x_{0}^{*}, x_{1}^{*}\right),
\end{gathered}
$$


и в силу этого допускает оценку

$$
\begin{gathered}
\|K(x, s)\| \leqslant \frac{M_{1}}{\gamma_{0}\left(x, x_{0}^{*}\right)}+\frac{M_{2}}{\gamma_{0}\left(s, x_{0}^{*}\right)} \leqslant M_{3} \varepsilon^{-1 / 4}, \quad \bar{x}_{*}(\varepsilon) \leqslant s \leqslant x \leqslant \overline{\bar{x}}_{*}(\varepsilon), \\
M_{j}=\mathrm{const}>0, \quad j=1,2,3 .
\end{gathered}
$$

В случае матрицы Коши $K(x, s, \varepsilon)$ системы (4.44) имеем дело с аналогичным (4.39) интегральным уравнением

$$
K(x, s, \varepsilon)=K(x, s)+\int_{s}^{x} K(x, \sigma)\left[A(\sigma, \varepsilon)-A_{0}(\sigma)\right] K(\sigma, s, \varepsilon) d \sigma,
$$

из которого, опираясь на свойства $(4.45),(4.46)$, последовательно выводим

$$
\begin{gathered}
\|K(x, s, \varepsilon)\| \leqslant M_{1} \varepsilon^{-1 / 4}+\int_{s}^{x}\left(\frac{M_{2} \varepsilon^{1 / 4}}{\gamma_{0}^{2}\left(\sigma, x_{0}^{*}\right)}+M_{3}\right)\|K(\sigma, s, \varepsilon)\| d \sigma \\
\|K(x, s, \varepsilon)\| \leqslant M_{1} \varepsilon^{-1 / 4} \exp \left[\int_{s}^{x}\left(\frac{M_{2} \varepsilon^{1 / 4}}{\gamma_{0}^{2}\left(\sigma, x_{0}^{*}\right)}+M_{3}\right) d \sigma\right] .
\end{gathered}
$$

Затем, учитывая в (4.47) вытекающее из (2.7), (4.43) неравенство

$$
\max _{\bar{x}_{*}(\varepsilon) \leqslant s \leqslant x \leqslant \bar{x}_{*}(\varepsilon)} \int_{s}^{x}\left(\frac{M_{2} \varepsilon^{1 / 4}}{\gamma_{0}^{2}\left(\sigma, x_{0}^{*}\right)}+M_{3}\right) d \sigma \leqslant M, \quad M=\text { const }>0,
$$

приходим к выводу, что

$$
\max _{\bar{x}_{*}(\varepsilon) \leqslant s \leqslant x \leqslant \overline{\bar{x}}_{*}(\varepsilon)}\|K(x, s, \varepsilon)\| \leqslant M \varepsilon^{-1 / 4}, \quad M=\text { const }>0 .
$$

И, наконец, вспоминая о сделанных ранее заменах (4.42), для матрицы Коши $K(t, \tau, \varepsilon)$ исходной системы (4.30) из (4.48) получаем оценку

$$
\max _{\tau_{*}^{(1)}(\varepsilon) \leqslant \tau \leqslant t \leqslant \tau_{*}^{(2)}(\varepsilon)}\|K(t, \tau, \varepsilon)\| \leqslant M \varepsilon^{-5 / 4}, \quad M=\text { const }>0 .
$$

Завершая анализ отрезка $\tau_{*}^{(1)}(\varepsilon) \leqslant t \leqslant \tau_{*}^{(2)}(\varepsilon)$, отметим, что на нем для $z(t, \varepsilon)$ справедлива формула вида (4.41), в которой начальный момент времени $t=0$ заменен на $t=\tau_{*}^{(1)}(\varepsilon)$. А отсюда и из (4.31), (4.49) вытекает справедливость требуемой оценки (4.35) на данном промежутке времени.

Случай $t \in\left[\tau_{*}^{(2)}(\varepsilon), t_{*}(\varepsilon)\right]$, где $t_{*}(\varepsilon)$ - момент времени (4.17), не нуждается в отдельном рассмотрении, так как по существу сводится к уже разобранной ситуации $0 \leqslant t \leqslant \tau_{*}^{(1)}(\varepsilon)$. Действительно, при указанных $t$ после замены времени

$$
t=\left.\theta_{x_{0}, \varphi}^{(3)}(y, \varepsilon)\right|_{x_{0}=x_{0}^{*}(\varepsilon), \varphi=\varphi_{*}}, \quad y \in\left[1, \varepsilon^{-3 / 4}\right],
$$

где $\theta_{x_{0}, \varphi}^{(3)}(y, \varepsilon)$ - функция из (3.104), система (4.30) преобразуется к виду (4.36), (4.37). Таким образом, оценка $(4.35)$ при $\tau_{*}^{(2)}(\varepsilon) \leqslant t \leqslant t_{*}(\varepsilon)$ заведомо сохраняется. 
На оставшемся отрезке времени $t_{*}(\varepsilon) \leqslant t \leqslant T_{*}(\varepsilon)-\delta$ в силу аналогичного (4.32) неравенства (см. (2.24), (3.106), (3.92), (3.94))

$$
\int_{t_{*}(\varepsilon)}^{T_{*}(\varepsilon)-\delta} y_{*}(t, \varepsilon) d t=-\varepsilon \int_{0}^{1} \frac{d y}{g\left(x_{1}^{*}, y\right)}+O\left(\varepsilon^{5 / 4}\right) \leqslant M \varepsilon, \quad M=\text { const }>0,
$$

и формулы (4.18) имеем

$$
\begin{gathered}
\max _{t_{*}(\varepsilon) \leqslant \tau \leqslant t \leqslant T_{*}(\varepsilon)-\delta}\|K(t, \tau, \varepsilon)\| \leqslant M_{1}, \quad \int_{h-\delta}^{T_{*}(\varepsilon)-\delta}\|B(t, \varepsilon)\| d t \leqslant \frac{M_{2}}{\varepsilon}, \\
M_{1}, M_{2}=\text { const }>0 .
\end{gathered}
$$

В свою очередь, свойства (4.50) позволяют для распространения оценки (4.35) на данный промежуток изменения $t$ воспользоваться следующим индуктивным процессом.

Разобьем отрезок $\left[t_{*}(\varepsilon), T_{*}(\varepsilon)-\delta\right]$ на фрагменты

$$
\begin{gathered}
{\left[t_{*}(\varepsilon)+k h, t_{*}(\varepsilon)+(k+1) h\right], \quad k=0,1, \ldots, k_{0},} \\
{\left[t_{*}(\varepsilon)+\left(k_{0}+1\right) h, T_{*}(\varepsilon)-\delta\right], \quad k_{0}=\left\lfloor\frac{T_{*}(\varepsilon)-t_{*}(\varepsilon)-h-\delta}{h}\right\rfloor,}
\end{gathered}
$$

$\lfloor *\rfloor$ - целая часть. Опираясь, далее, на соотношения (4.31), (4.50), замечаем, что из неравенства

$$
\begin{aligned}
\|z(t, \varepsilon)\| \leqslant & \left\|K\left(t, t_{*}(\varepsilon)+k h, \varepsilon\right)\right\| \cdot\left\|z\left(t_{*}(\varepsilon)+k h, \varepsilon\right)\right\| \\
& +\int_{t_{*}(\varepsilon)+k h}^{t}\|K(t, \tau, \varepsilon)\| \cdot\|B(\tau, \varepsilon)\| \cdot\|z(\tau-h, \varepsilon)\| d \tau, \quad t \geqslant t_{*}(\varepsilon)+k h,
\end{aligned}
$$

и из уже полученной оценки вида $(4.35)$ на $(k-1)$-м отрезке вытекает требуемая оценка на $k$-м отрезке изменения $t$. Лемма 4.1 доказана.

Приступим теперь ко второму этапу исследования мультипликаторов системы (4.20). На нем мы изучим спектральные свойства двумерного оператора $V(\varepsilon): E \rightarrow E$, результат действия которого на любую функцию $z_{0}(t) \in E$ задается равенством

$$
\begin{aligned}
V(\varepsilon) z_{0}= & z_{1}^{0} \operatorname{colon}\left(z_{1}^{(1)}\left(T_{*}(\varepsilon)-\delta, \varepsilon\right), z_{2}^{(1)}\left(t+T_{*}(\varepsilon), \varepsilon\right)\right) \\
& +z_{2}^{0}(-\delta) \operatorname{colon}\left(z_{1}^{(2)}\left(T_{*}(\varepsilon)-\delta, \varepsilon\right), z_{2}^{(2)}\left(t+T_{*}(\varepsilon), \varepsilon\right)\right), \quad-h-\delta \leqslant t \leqslant-\delta .
\end{aligned}
$$

Здесь через

$$
z^{(j)}(t, \varepsilon)=\operatorname{colon}\left(z_{1}^{(j)}(t, \varepsilon), z_{2}^{(j)}(t, \varepsilon)\right), \quad-\delta \leqslant t \leqslant T_{*}(\varepsilon)-\delta, \quad j=1,2,
$$

обозначены решения системы (4.20) с начальными условиями, заданными при $-h-\delta \leqslant t \leqslant-\delta$ равенствами $z_{0}(t) \equiv \operatorname{colon}(1,0)$ и $z_{0}(t) \equiv \operatorname{colon}(0,1)$ соответственно. 
Остановимся на вопросе о связи между операторами (4.24) и (4.51). С этой целью обратим внимание, что фигурирующее в (4.24) решение $z(t, \varepsilon)$ может быть представлено в виде

$$
z(t, \varepsilon)=z_{1}^{0} z^{(1)}(t, \varepsilon)+z_{2}^{0}(-\delta) z^{(2)}(t, \varepsilon)+\omega(t, \varepsilon), \quad-\delta \leqslant t \leqslant T_{*}(\varepsilon)-\delta,
$$

где $\omega(t, \varepsilon)$ - решение системы (4.20) с начальным условием

$$
\omega_{0}(t)=\operatorname{colon}\left(0, z_{2}^{0}(t)-z_{2}^{0}(-\delta)\right) \in E_{0}, \quad-h-\delta \leqslant t \leqslant-\delta .
$$

Таким образом, третье слагаемое в (4.53) в силу леммы 4.1 удовлетворяет оценке вида (4.28). А отсюда очевидным образом следует, что для оператоpa $W(\varepsilon)=U(\varepsilon)-V(\varepsilon)$ справедливо неравенство

$$
\|W(\varepsilon)\|_{E \rightarrow E} \leqslant M_{1} \exp \left(-\frac{M_{2}}{\varepsilon}\right), \quad M_{1}, M_{2}=\text { const }>0 .
$$

Нетрудно увидеть, что спектр оператора (4.51) состоит из собственных значений двумерной матрицы

$$
\mathscr{D}(\varepsilon)=\left.\left[z^{(1)}(t, \varepsilon), z^{(2)}(t, \varepsilon)\right]\right|_{t=T_{*}(\varepsilon)-\delta}
$$

и из нулевого собственного значения бесконечной кратности. Тем самым, проблема спектрального анализа данного оператора сводится к асимптотическому вычислению матрицы (4.55).

Лемма 4.2. Найдется такое достаточно малое $\varepsilon_{0}>0$, что при всех $0<$ $\varepsilon \leqslant \varepsilon_{0}$ справедливы следующие факты. Во-первых, для оператора (4.51) имеет место оиенка

$$
\|V(\varepsilon)\|_{E \rightarrow E} \leqslant \frac{M}{\varepsilon}, \quad M=\text { const }>0 ;
$$

во-вторых, собственные значения $\lambda_{1}(\varepsilon), \lambda_{2}(\varepsilon)$ матрицы (4.55) при $\varepsilon \rightarrow 0$ допускают асимптотику

$$
\lambda_{1}(\varepsilon)=1+O(\sqrt{\varepsilon}), \quad \lambda_{2}(\varepsilon)=\psi^{\prime}\left(x_{0}^{*}\right)+O(\sqrt{\varepsilon}) .
$$

ДокАЗАТЕЛЬСтво. Проблема обоснования соотношений (4.56), (4.57) очевидным образом связана с построением асимптотики введенных выше решений (4.52). Однако соответствующий асимптотический анализ мы проведем сначала для двух других решений

$$
\varkappa^{(j)}(t, \varepsilon)=\operatorname{colon}\left(\varkappa_{1}^{(j)}(t, \varepsilon), \varkappa_{2}^{(j)}(t, \varepsilon)\right), \quad-\delta \leqslant t \leqslant T_{*}(\varepsilon)-\delta, \quad j=1,2,
$$

системы (4.20), а потом представим нужные нам решения (4.52) в виде линейных комбинаций вектор-функций (4.58). Что же касается начальных условий для решений (4.58), то их при $-h-\delta \leqslant t \leqslant-\delta$ зададим равенствами

$$
\begin{gathered}
\left.\varkappa^{(1)}(t, \varepsilon) \equiv \operatorname{colon}\left(\dot{x}_{*}(t, \varepsilon), \frac{\varepsilon \dot{y}_{*}(t, \varepsilon)}{y_{*}(t, \varepsilon)}\right)\right|_{t=-\delta} \\
=\left.\operatorname{colon}(f(x(-\delta, s), 0), g(x(-\delta, s), 0))\right|_{s=x_{0}^{*}}+O(\sqrt{\varepsilon}), \\
\left.\varkappa^{(2)}(t, \varepsilon) \equiv \operatorname{colon}\left(\frac{\partial x}{\partial x_{0}}\left(t, x_{0}, \varepsilon\right), \varepsilon \frac{\partial y}{\partial x_{0}}\left(t, x_{0}, \varepsilon\right) / y\left(t, x_{0}, \varepsilon\right)\right)\right|_{t=-\delta, x_{0}=x_{0}^{*}(\varepsilon)},
\end{gathered}
$$


где, напомним, $x(t, s)$ - решение задачи $(3.122), x\left(t, x_{0}, \varepsilon\right), y\left(t, x_{0}, \varepsilon\right)-$ функции из (3.9).

Вопрос об асимптотике решения $\varkappa^{(1)}(t, \varepsilon)$ не вызывает затруднений. Действительно, учитывая способ получения системы (4.20) и, в частности, замену (4.19), нетрудно увидеть, что ее решением является вектор-функция

$$
\operatorname{colon}\left(\dot{x}_{*}(t, \varepsilon), \frac{\varepsilon \dot{y}_{*}(t, \varepsilon)}{y_{*}(t, \varepsilon)}\right), \quad-\delta \leqslant t \leqslant T_{*}(\varepsilon)-\delta .
$$

Заметим далее, что решение (4.61) порождается начальным условием

$$
\operatorname{colon}\left(\dot{x}_{*}(-\delta, \varepsilon), \frac{\varepsilon \dot{y}_{*}(t, \varepsilon)}{y_{*}(t, \varepsilon)}\right), \quad-h-\delta \leqslant t \leqslant-\delta,
$$

причем, что самое главное, разность между (4.59) и (4.62) принадлежит пространству $E_{0}$ и ограничена по норме (4.23) равномерно относительно $\varepsilon$. А отсюда и из леммы 4.1 следует, что при $\varepsilon \rightarrow 0$ имеет место равномерное по $-\delta \leqslant t \leqslant T_{*}(\varepsilon)-\delta$ асимптотическое представление

$$
\begin{gathered}
\varkappa^{(1)}(t, \varepsilon)=\operatorname{colon}\left(\dot{x}_{*}(t, \varepsilon), \frac{\varepsilon \dot{y}_{*}(t, \varepsilon)}{y_{*}(t, \varepsilon)}\right)+O\left(\exp \left(-\frac{M}{\varepsilon}\right)\right), \\
M=\text { const }>0 .
\end{gathered}
$$

Для выявления асимптотических свойств решения $\varkappa^{(2)}(t, \varepsilon)$ необходимо знать асимптотику функций $x\left(t, x_{0}, \varepsilon\right), y\left(t, x_{0}, \varepsilon\right)$ из $(3.9)$ на отрезке $-\delta \leqslant t \leqslant 0$. Соответствующий анализ проводится с помощью развитых в предыдущем параграфе геометрических методов. Поэтому здесь ограничимся лишь сводкой итоговых результатов.

Обратимся сначала к отрезку $\tau_{0}\left(x_{0}, \varepsilon\right) \leqslant t \leqslant 0$, где $\tau_{0}\left(x_{0}, \varepsilon\right)-$ наибольший отрицательный корень уравнения $y\left(t, x_{0}, \varepsilon\right)=\exp \left(-\varepsilon^{-3 / 4}\right)$, и на кривой

$$
\Lambda_{0}(\varepsilon)=\left\{(t, x, y): \tau_{0}\left(x_{0}, \varepsilon\right) \leqslant t \leqslant 0, x=x\left(t, x_{0}, \varepsilon\right), y=y\left(t, x_{0}, \varepsilon\right)\right\}
$$

в качестве параметра возьмем переменную $y \in\left[\exp \left(-\varepsilon^{-3 / 4}\right), 1\right]$. В итоге убеждаемся (см. обоснование лемм $3.1,3.5)$, что для $\Lambda_{0}(\varepsilon)$ справедливо аналогичное (3.11), (3.90) равенство

$$
\Lambda_{0}(\varepsilon)=\left\{(t, x, y): t=\theta_{0}\left(y, x_{0}, \varepsilon\right), x=x\left(y, x_{0}, \varepsilon\right), \exp \left(-\varepsilon^{-3 / 4}\right) \leqslant y \leqslant 1\right\},
$$

где функции $\theta_{0}\left(y, x_{0}, \varepsilon\right), x\left(y, x_{0}, \varepsilon\right)$ допускают при $\varepsilon \rightarrow 0$ равномерные по начальному условию $x_{0} \in[a, b]$ и по $y \in\left[\exp \left(-\varepsilon^{-3 / 4}\right), 1\right]$ асимптотические представления

$$
\begin{gathered}
\theta_{0}\left(y, x_{0}, \varepsilon\right)=\varepsilon \int_{1}^{y} \frac{d s}{s g\left(x_{0}, s\right)}+O\left(\varepsilon^{5 / 4} \ln \frac{1}{y}\right) \\
\frac{\partial}{\partial x_{0}} \theta_{0}\left(y, x_{0}, \varepsilon\right)=\varepsilon \frac{\partial}{\partial x_{0}}\left(\int_{1}^{y} \frac{d s}{s g\left(x_{0}, s\right)}\right)+O\left(\varepsilon^{5 / 4} \ln \frac{1}{y}\right) \\
\frac{\partial \theta_{0}}{\partial y}\left(y, x_{0}, \varepsilon\right)=\frac{\varepsilon}{y}\left(\frac{1}{g\left(x_{0}, y\right)}+O\left(\varepsilon^{1 / 4}\right)\right)
\end{gathered}
$$




$$
\begin{gathered}
\frac{\partial^{2}}{\partial x_{0} \partial y} \theta_{0}\left(y, x_{0}, \varepsilon\right)=\frac{\varepsilon}{y}\left(\frac{\partial}{\partial x_{0}}\left(\frac{1}{g\left(x_{0}, y\right)}\right)+O\left(\varepsilon^{1 / 4}\right)\right), \\
x\left(y, x_{0}, \varepsilon\right)=x_{0}+\varepsilon \int_{1}^{y} \frac{f\left(x_{0}, s\right)}{s g\left(x_{0}, s\right)} d s+O\left(\varepsilon^{5 / 4} \ln \frac{1}{y}\right), \\
\frac{\partial}{\partial y} x\left(y, x_{0}, \varepsilon\right)=O(\varepsilon / y), \\
\frac{\partial}{\partial x_{0}} x\left(y, x_{0}, \varepsilon\right)=1+\varepsilon \int_{1}^{y} \frac{\partial}{\partial x_{0}}\left(\frac{f\left(x_{0}, s\right)}{s g\left(x_{0}, s\right)}\right) d s+O\left(\varepsilon^{5 / 4} \ln \frac{1}{y}\right) .
\end{gathered}
$$

Что же касается фигурирующего в (4.64) момента времени $\tau_{0}\left(x_{0}, \varepsilon\right)$, то для него из (4.66), (4.67) выводим

$$
\begin{gathered}
\left.\tau_{0}\left(x_{0}, \varepsilon\right) \stackrel{\text { def }}{=} \theta_{0}\left(y, x_{0}, \varepsilon\right)\right|_{y=\exp \left(-\varepsilon^{-3 / 4}\right)}=-\frac{\varepsilon^{1 / 4}}{g\left(x_{0}, 0\right)}+O(\sqrt{\varepsilon}), \\
\frac{\partial}{\partial x_{0}} \tau_{0}\left(x_{0}, \varepsilon\right)=-\frac{\partial}{\partial x_{0}}\left(\frac{\varepsilon^{1 / 4}}{g\left(x_{0}, 0\right)}\right)+O(\sqrt{\varepsilon}) .
\end{gathered}
$$

В случае $-\delta \leqslant t \leqslant \tau_{0}\left(x_{0}, \varepsilon\right)$, рассуждая как и при доказательстве леммы 3.7, для $x\left(t, x_{0}, \varepsilon\right), v\left(t, x_{0}, \varepsilon\right)=\varepsilon \ln y\left(t, x_{0}, \varepsilon\right)$ приходим к аналогичным $(3.119),(3.120)$ асимптотическим равенствам

$$
\begin{gathered}
x\left(t, x_{0}, \varepsilon\right)=\left.x(t, s)\right|_{s=x_{0}}+O(\sqrt{\varepsilon}), \\
\frac{\partial x}{\partial x_{0}}\left(t, x_{0}, \varepsilon\right)=\left.\frac{f(x(t, s), 0)}{f(s, 0)}\right|_{s=x_{0}}+O(\sqrt{\varepsilon}), \\
v\left(t, x_{0}, \varepsilon\right)=\left.\int_{0}^{t} g(x(\tau, s), 0) d \tau\right|_{s=x_{0}}+O(\sqrt{\varepsilon}), \\
\frac{\partial v}{\partial x_{0}}\left(t, x_{0}, \varepsilon\right)=\left.\frac{g(x(t, s), 0)-g(s, 0)}{f(s, 0)}\right|_{s=x_{0}}+O(\sqrt{\varepsilon}),
\end{gathered}
$$

которые имеют место равномерно по $x_{0} \in[a, b]$ и $t$.

Помимо формул $(4.65)-(4.73)$ при анализе решения $\varkappa^{(2)}(t, \varepsilon)$ нам потребуется следующая дополнительная информация. А именно, ниже будут использованы равномерные по $t \in[-\delta, h-\delta]$ асимптотические представления

$$
\begin{gathered}
x_{*}(t, \varepsilon)=\left.x\left(t, x_{0}, \varepsilon\right)\right|_{x_{0}=x_{0}^{*}(\varepsilon)}+O\left(\exp \left(-\frac{M}{\varepsilon}\right)\right), \\
y_{*}(t, \varepsilon)=\left.\left(1+O\left(\exp \left(-\frac{M}{\varepsilon}\right)\right)\right) y\left(t, x_{0}, \varepsilon\right)\right|_{x_{0}=x_{0}^{*}(\varepsilon)},
\end{gathered}
$$

где $M=$ const $>0$. Заметим, что при $t \in[0, h-\delta]$ формулы (4.74) вытекают из (3.136). В случае же $t \in[-\delta, 0]$ учтем тот факт, что кривая (3.164) аппроксимируется с экспоненциальной точностью фазовой кривой, порожденной задачей Коши (3.165). Полагая в (3.164), (3.165) $x_{0}=x_{0}^{*}(\varepsilon), \varphi=\varphi_{*}(t, \varepsilon)($ см. (4.12)) и обыгрывая свойство периодичности функций $x_{*}(t, \varepsilon), y_{*}(t, \varepsilon)$, приходим к требуемым соотношениям (4.74) при $t \in[-\delta, 0]$. 
Обозначим через $\widetilde{A}(t, \varepsilon)$ матрицу, получающуюся из $A(t, \varepsilon)$ при замене в $(4.22)$ компонент $x_{*}(t, \varepsilon), y_{*}(t, \varepsilon)$ на $\left.\left(x\left(t, x_{0}, \varepsilon\right), y\left(t, x_{0}, \varepsilon\right)\right)\right|_{x_{0}=x_{0}^{*}(\varepsilon)}$. Заметим далее, что вектор-функция

$$
\widetilde{z}(t, \varepsilon)=\left.\operatorname{colon}\left(\frac{\partial x}{\partial x_{0}}\left(t, x_{0}, \varepsilon\right), \varepsilon \frac{\partial y}{\partial x_{0}}\left(t, x_{0}, \varepsilon\right) / y\left(t, x_{0}, \varepsilon\right)\right)\right|_{x_{0}=x_{0}^{*}(\varepsilon)}
$$

удовлетворяет системе $\dot{z}=\widetilde{A}(t, \varepsilon) z$. А отсюда и из формулы (4.60) следует, что разность $\Delta(t, \varepsilon)=\varkappa^{(2)}(t, \varepsilon)-\widetilde{z}(t, \varepsilon)$ при $t \in[-\delta, h-\delta]$ определяется из задачи Коши

$$
\dot{\Delta}-A(t, \varepsilon) \Delta=B(t, \varepsilon) \varkappa^{(2)}(t-h, \varepsilon)+[A(t, \varepsilon)-\widetilde{A}(t, \varepsilon)] \widetilde{z}(t, \varepsilon),\left.\quad \Delta\right|_{t=-\delta}=0
$$

и в силу этого задается равенством

$$
\Delta(t, \varepsilon)=\int_{-\delta}^{t} K(t, \tau, \varepsilon)\left[B(\tau, \varepsilon) \varkappa^{(2)}(\tau-h, \varepsilon)+[A(\tau, \varepsilon)-\widetilde{A}(\tau, \varepsilon)] \widetilde{z}(\tau, \varepsilon)\right] d \tau .
$$

Покажем теперь, что неоднородность из (4.76), которая, подчеркнем, при $t \in[-\delta, h-\delta]$ нам известна, является экспоненциально малой. В связи с этим обратим внимание, что из соотношений (4.31), (4.60), (4.72)-(4.74) вытекает неравенство

$$
\begin{gathered}
\max _{-\delta \leqslant t \leqslant h-\delta}\left\|B(t, \varepsilon) \varkappa^{(2)}(t-h, \varepsilon)\right\|+\max _{-\delta \leqslant t \leqslant h-\delta}\|A(t, \varepsilon)-\widetilde{A}(t, \varepsilon)\| \leqslant M_{1} \exp \left(-\frac{M_{2}}{\varepsilon}\right), \\
M_{1}, M_{2}=\mathrm{const}>0 .
\end{gathered}
$$

Что же касается функции (4.75), то для ее оценки привлечем уже известную информацию об асимптотическом поведении $x\left(t, x_{0}, \varepsilon\right), y\left(t, x_{0}, \varepsilon\right), \partial x\left(t, x_{0}, \varepsilon\right) / \partial x_{0}$, $\partial y\left(t, x_{0}, \varepsilon\right) / \partial x_{0}$ (см. леммы 3.1, 3.3, 3.5, 3.7 и формулы $\left.(4.65)-(4.73)\right)$. В результате убеждаемся, что

$$
\max _{-\delta \leqslant t \leqslant h-\delta}\|\widetilde{z}(t, \varepsilon)\| \leqslant M \varepsilon^{-1 / 4}, \quad M=\text { const }>0
$$

самый худший вариант реализуется на участке поворота, где в силу (3.53), (3.54) имеем

$$
\frac{\partial x}{\partial x_{0}}=-\left(\frac{\partial \theta_{2}}{\partial x}\right)^{-1} \frac{\partial \theta_{2}}{\partial x_{0}}=O\left(\varepsilon^{-1 / 4}\right) .
$$

Подведем промежуточный итог. Из соотношений (4.77)-(4.79) и из известных оценок на матрицу Коши $K(t, \tau, \varepsilon),-\delta \leqslant \tau \leqslant t \leqslant h-\delta$ (см. (4.33), (4.40), (4.49), (4.50)), следует, что при $\varepsilon \rightarrow 0$ равномерно по $t \in[-\delta, h-\delta]$

$$
\varkappa^{(2)}(t, \varepsilon)=\widetilde{z}(t, \varepsilon)+O\left(\exp \left(-\frac{M}{\varepsilon}\right)\right), \quad M=\text { const }>0,
$$

где $\widetilde{z}(t, \varepsilon)$ - функция (4.75). 
При построении асимптотики решения $\varkappa^{(2)}(t, \varepsilon)$ на оставшемся отрезке времени $h-\delta \leqslant t \leqslant T_{*}(\varepsilon)-\delta$ система (4.20) интегрируется методом шагов, т.е. последовательно рассматривается на промежутках длины не более $h$. В процессе ее анализа существенно используются вытекающие из (2.24) оценки

$$
\begin{gathered}
\max _{h-\delta \leqslant t \leqslant T_{*}(\varepsilon)-\delta}\left(\left|a_{11}(t, \varepsilon)-f_{x}^{\prime}\left(x_{*}(t, \varepsilon), 0\right)\right|+\left|a_{21}(t, \varepsilon)-g_{x}^{\prime}\left(x_{*}(t, \varepsilon), 0\right)\right|\right. \\
\left.+\left|a_{12}(t, \varepsilon)\right|+\left|a_{22}(t, \varepsilon)\right|\right) \leqslant M_{1} \exp \left(-\frac{M_{2}}{\varepsilon}\right), \\
\max _{h+\delta \leqslant t \leqslant T_{*}(\varepsilon)-\delta}\|B(t, \varepsilon)\| \leqslant M_{3} \exp \left(-\frac{M_{4}}{\varepsilon}\right),
\end{gathered}
$$

где $M_{j}=$ const $>0, j=1, \ldots, 4$.

На отрезке $h-\delta \leqslant t \leqslant h+\delta$ в силу (4.80), (4.81) для отыскания компонент $z_{1}=\varkappa_{1}^{(2)}(t, \varepsilon), z_{2}=\varkappa_{2}^{(2)}(t, \varepsilon)$ решения $\varkappa^{(2)}(t, \varepsilon)$ с точностью до экспоненциально малых добавок получаем задачу Коши

$$
\begin{gathered}
\dot{z}_{1}=f_{x}^{\prime}\left(x_{*}(t, \varepsilon), 0\right) z_{1}, \quad \dot{z}_{2}=g_{x}^{\prime}\left(x_{*}(t, \varepsilon), 0\right) z_{1}-\left.\frac{\partial y}{\partial x_{0}}\left(t-h, x_{0}, \varepsilon\right)\right|_{x_{0}=x_{0}^{*}(\varepsilon)}, \\
\left.z_{1}\right|_{t=h-\delta}=\left.\frac{\partial x}{\partial x_{0}}\left(h-\delta, x_{0}, \varepsilon\right)\right|_{x_{0}=x_{0}^{*}(\varepsilon)}, \\
\left.z_{2}\right|_{t=h-\delta}=\varepsilon \frac{\partial y}{\partial x_{0}}\left(h-\delta, x_{0}, \varepsilon\right) /\left.y\left(h-\delta, x_{0}, \varepsilon\right)\right|_{x_{0}=x_{0}^{*}(\varepsilon)} .
\end{gathered}
$$

Учитывая, далее, в (4.83), (4.84) уже известные асимптотические представления для функций $x_{*}(t, \varepsilon)=\left.x_{\left(x_{0}, \varphi\right)}(t, \varepsilon)\right|_{x_{0}=x_{0}^{*}(\varepsilon), \varphi=\varphi_{*}}, x\left(t, x_{0}, \varepsilon\right), y\left(t, x_{0}, \varepsilon\right), x_{0}^{*}(\varepsilon)$ (см. леммы 3.7, 3.8 и последующие формулы (3.142), (4.15)), приходим к выводу, что при $\varepsilon \rightarrow 0$ равномерно по $t \in[h-\delta, h+\delta]$

$$
\begin{gathered}
\varkappa_{1}^{(2)}(t, \varepsilon)=\left.\frac{f(x(t, s), 0)}{f(s, 0)}\right|_{s=x_{1}^{*}} \cdot x_{1}^{\prime}\left(x_{0}^{*}\right)+O(\sqrt{\varepsilon}), \\
\varkappa_{2}^{(2)}(t, \varepsilon)=\left.\frac{g(x(t, s), 0)-g(s, 0)}{f(s, 0)}\right|_{s=x_{1}^{*}} \cdot x_{1}^{\prime}\left(x_{0}^{*}\right) \\
\quad-\left.\frac{\partial}{\partial x_{0}}\left(\int_{-\delta}^{t-h} y\left(\tau, x_{0}, \varepsilon\right) d \tau\right)\right|_{x_{0}=x_{0}^{*}(\varepsilon)}+O(\sqrt{\varepsilon}),
\end{gathered}
$$

где, напомним, $x_{1}^{*}=\left.x_{1}\left(x_{0}\right)\right|_{x_{0}=x_{0}^{*}}$.

Отдельного рассмотрения требует интегральное слагаемое из (4.86), анализ которого аналогичен исследованию соответствующего интегрального слагаемого из (3.142). В данном случае обозначим через $I_{k}(x, \varepsilon), J_{k}(x, \varepsilon), k=1, \ldots, 6$, интегралы от функций $y\left(t, x_{0}, \varepsilon\right),\left|\partial y\left(t, x_{0}, \varepsilon\right) / \partial x_{0}\right|$ по отрезкам

$$
\begin{gathered}
{\left[-\delta, \tau_{0}\left(x_{0}, \varepsilon\right)\right], \quad\left[\tau_{0}\left(x_{0}, \varepsilon\right), 0\right], \quad\left[0, \tau_{1}\left(x_{0}, \varepsilon\right)\right], \quad\left[\tau_{1}\left(x_{0}, \varepsilon\right), \tau_{2}\left(x_{0}, \varepsilon\right)\right],} \\
{\left[\tau_{2}\left(x_{0}, \varepsilon\right), \tau_{3}\left(x_{0}, \varepsilon\right)\right], \quad\left[\tau_{3}\left(x_{0}, \varepsilon\right), \delta\right]}
\end{gathered}
$$

и проведем их асимптотическое вычисление. 
Рассмотрим сначала наиболее простые варианты $k=1,6$ из (4.87) и заметим, что при

$$
t \in\left[-\delta, \tau_{0}\left(x_{0}, \varepsilon\right)\right] \cup\left[\tau_{3}\left(x_{0}, \varepsilon\right), \delta\right]
$$

в силу (3.118)-(3.120), (4.72), (4.73) имеют место оценки

$$
\begin{gathered}
y\left(t, x_{0}, \varepsilon\right) \leqslant M_{1} \exp \left(-\varepsilon^{-3 / 4}\right), \quad\left|\frac{\partial y}{\partial x_{0}}\left(t, x_{0}, \varepsilon\right)\right| \leqslant \frac{M_{2}}{\varepsilon} \exp \left(-\varepsilon^{-3 / 4}\right), \\
M_{1}, M_{2}=\text { const }>0 .
\end{gathered}
$$

А отсюда и из (3.114), (3.115), (4.71) очевидным образом следует, что

$$
\begin{gathered}
I_{1}\left(x_{0}, \varepsilon\right)+I_{6}\left(x_{0}, \varepsilon\right)+\left|\frac{\partial I_{1}}{\partial x_{0}}\left(x_{0}, \varepsilon\right)\right|+\left|\frac{\partial I_{6}}{\partial x_{0}}\left(x_{0}, \varepsilon\right)\right|+J_{1}\left(x_{0}, \varepsilon\right)+J_{6}\left(x_{0}, \varepsilon\right) \\
\leqslant \frac{M}{\varepsilon} \exp \left(-\varepsilon^{-3 / 4}\right), \quad M=\text { const }>0 .
\end{gathered}
$$

В случаях $k=2,3,5$ справедливы аналогичные (3.144), (3.146) формулы

$$
\begin{aligned}
& I_{2}\left(x_{0}, \varepsilon\right)=\int_{\exp \left(-\varepsilon^{-3 / 4}\right)}^{1} y \frac{\partial}{\partial y} \theta_{0}\left(y, x_{0}, \varepsilon\right) d y=O(\varepsilon), \quad \frac{\partial I_{2}}{\partial x_{0}}\left(x_{0}, \varepsilon\right)=O(\varepsilon), \\
& J_{2}\left(x_{0}, \varepsilon\right)=\int_{\exp \left(-\varepsilon^{-3 / 4}\right)}^{1}\left|\frac{\partial \theta_{0}}{\partial x_{0}}\left(y, x_{0}, \varepsilon\right)\right| d y=O(\varepsilon), \\
& I_{3}\left(x_{0}, \varepsilon\right)=\int_{1}^{\varepsilon^{-3 / 4}} y \frac{\partial}{\partial y} \theta_{1}\left(y, x_{0}, \varepsilon\right) d y=\frac{\varepsilon^{1 / 4}}{b_{0}\left(x_{0}\right)}+O(\sqrt{\varepsilon}), \\
& \frac{\partial I_{3}}{\partial x_{0}}\left(x_{0}, \varepsilon\right)=\frac{\partial}{\partial x_{0}}\left(\frac{\varepsilon^{1 / 4}}{b_{0}\left(x_{0}\right)}\right)+O(\sqrt{\varepsilon}), \\
& J_{3}\left(x_{0}, \varepsilon\right)=\int_{1}^{\varepsilon^{-3 / 4}}\left|\frac{\partial \theta_{1}}{\partial x_{0}}\left(y, x_{0}, \varepsilon\right)\right| d y=O\left(\varepsilon^{1 / 4} \ln \frac{1}{\varepsilon}\right), \\
& I_{5}\left(x_{0}, \varepsilon\right)=\int_{\varepsilon^{-3 / 4}}^{\exp \left(-\varepsilon^{-3 / 4}\right)} y \frac{\partial}{\partial y} \theta_{3}\left(y, x_{0}, \varepsilon\right) d y=-\frac{\varepsilon^{1 / 4}}{b_{0}\left(x_{1}\left(x_{0}\right)\right)}+O(\sqrt{\varepsilon}), \\
& \frac{\partial I_{5}}{\partial x_{0}}\left(x_{0}, \varepsilon\right)=-\frac{\partial}{\partial x_{0}}\left(\frac{\varepsilon^{1 / 4}}{b_{0}\left(x_{1}\left(x_{0}\right)\right)}\right)+O(\sqrt{\varepsilon}), \\
& J_{5}\left(x_{0}, \varepsilon\right)=\int_{\exp \left(-\varepsilon^{-3 / 4}\right)}^{\varepsilon^{-3 / 4}}\left|\frac{\partial \theta_{3}}{\partial x_{0}}\left(y, x_{0}, \varepsilon\right)\right| d y=O\left(\varepsilon^{1 / 4} \ln \frac{1}{\varepsilon}\right),
\end{aligned}
$$

при выводе которых использовались известные свойства функций $\theta_{j}\left(y, x_{0}, \varepsilon\right)$, $j=0,1,3$ (см. (3.12)-(3.15), (3.92)-(3.95), (4.66)-(4.69)). И, наконец, при $k=4$, опираясь на соотношения (3.47)-(3.55), приходим к выводу, что

$$
\begin{aligned}
I_{4}\left(x_{0}, \varepsilon\right) & =\frac{1}{\varepsilon} \int_{\bar{x}\left(x_{0}, \varepsilon\right)}^{\overline{\bar{x}}\left(x_{0}, \varepsilon\right)} u\left(x, x_{0}, \varepsilon\right) \frac{d x}{f\left(x, u\left(x, x_{0}, \varepsilon\right) / \varepsilon\right)} \\
& =\int_{x_{0}}^{x_{1}\left(x_{0}\right)} \frac{d s}{a_{0}(s)}+\left(\frac{1}{b_{0}\left(x_{1}\left(x_{0}\right)\right)}-\frac{1}{b_{0}\left(x_{0}\right)}\right) \varepsilon^{1 / 4}+O(\sqrt{\varepsilon}),
\end{aligned}
$$




$$
\begin{gathered}
\frac{\partial I_{4}}{\partial x_{0}}\left(x_{0}, \varepsilon\right)=\frac{\partial}{\partial x_{0}}\left(\int_{x_{0}}^{x_{1}\left(x_{0}\right)} \frac{d s}{a_{0}(s)}+\left(\frac{1}{b_{0}\left(x_{1}\left(x_{0}\right)\right)}-\frac{1}{b_{0}\left(x_{0}\right)}\right) \varepsilon^{1 / 4}\right)+O(\sqrt{\varepsilon}), \\
J_{4}\left(x_{0}, \varepsilon\right)=\frac{1}{\varepsilon} \int_{\bar{x}\left(x_{0}, \varepsilon\right)}^{\overline{\bar{x}}\left(x_{0}, \varepsilon\right)}\left|\frac{\partial u}{\partial x_{0}}\left(x, x_{0}, \varepsilon\right) \frac{\partial \theta_{2}}{\partial x}\left(x, x_{0}, \varepsilon\right)-\frac{\partial u}{\partial x}\left(x, x_{0}, \varepsilon\right) \frac{\partial \theta_{2}}{\partial x_{0}}\left(x, x_{0}, \varepsilon\right)\right| d x \\
\leqslant M \varepsilon^{-1 / 4}, \quad M=\text { const }>0 .
\end{gathered}
$$

Просуммируем полученную информацию. Из соотношений (4.88)-(4.99) вытекает, что

$$
\begin{gathered}
\max _{h-\delta \leqslant t \leqslant h+\delta}\left|\left(\frac{\partial}{\partial x_{0}} \int_{-\delta}^{t-h} y\left(\tau, x_{0}, \varepsilon\right) d \tau\right)\right|_{x_{0}=x_{0}^{*}(\varepsilon)} \mid \leqslant M \varepsilon^{-1 / 4}, \quad M=\text { const }>0, \\
\left.\frac{\partial}{\partial x_{0}}\left(\int_{-\delta}^{\delta} y\left(\tau, x_{0}, \varepsilon\right) d \tau\right)\right|_{x_{0}=x_{0}^{*}(\varepsilon)}=\left.\frac{d}{d x_{0}}\left(\int_{x_{0}}^{x_{1}\left(x_{0}\right)} \frac{d s}{a_{0}(s)}\right)\right|_{x_{0}=x_{0}^{*}}+O(\sqrt{\varepsilon}),
\end{gathered}
$$

а значит, в силу (4.85), (4.86) имеем

$$
\begin{gathered}
\max _{h-\delta \leqslant t \leqslant h+\delta}\left\|\varkappa^{(2)}(t, \varepsilon)\right\| \leqslant M \varepsilon^{-1 / 4}, \quad M=\text { const }>0, \\
\varkappa_{1}^{(2)}(h+\delta, \varepsilon)=\left.\frac{f(x(h+\delta, s), 0)}{f(s, 0)}\right|_{s=x_{1}^{*}} \cdot x_{1}^{\prime}\left(x_{0}^{*}\right)+O(\sqrt{\varepsilon}), \\
\varkappa_{2}^{(2)}(h+\delta, \varepsilon)=\left.\frac{g(x(h+\delta, s), 0)-g(s, 0)}{f(s, 0)}\right|_{s=x_{1}^{*}} \cdot x_{1}^{\prime}\left(x_{0}^{*}\right) \\
-\left.\frac{d}{d x_{0}}\left(\int_{x_{0}}^{x_{1}\left(x_{0}\right)} \frac{d s}{a_{0}(s)}\right)\right|_{x_{0}=x_{0}^{*}}+O(\sqrt{\varepsilon}) .
\end{gathered}
$$

Заканчивая построение асимптотики решения $\varkappa^{(2)}(t, \varepsilon)$, рассмотрим отрезок времени $h+\delta \leqslant t \leqslant T_{*}(\varepsilon)-\delta$, на котором согласно (4.81), (4.82) система (4.20) с экспоненциальной точностью аппроксимируется системой

$$
\dot{z}_{1}=f_{x}^{\prime}\left(x_{*}(t, \varepsilon), 0\right) z_{1}, \quad \dot{z}_{2}=g_{x}^{\prime}\left(x_{*}(t, \varepsilon), 0\right) z_{1} .
$$

Дополняя последнюю начальными условиями (4.101), (4.102), приходим к выводу, что для $\varkappa_{1}^{(2)}(t, \varepsilon)$ сохраняется равномерное по $t \in\left[h+\delta, T_{*}(\varepsilon)-\delta\right]$ асимптотическое равенство $(4.85)$, а для $\varkappa_{2}^{(2)}(t, \varepsilon)$ равномерно по $t$ имеем

$$
\begin{aligned}
\varkappa_{2}^{(2)}(t, \varepsilon)= & \left.\frac{g(x(t, s), 0)-g(s, 0)}{f(s, 0)}\right|_{s=x_{1}^{*}} \cdot x_{1}^{\prime}\left(x_{0}^{*}\right) \\
& \quad-\left.\frac{d}{d x_{0}}\left(\int_{x_{0}}^{x_{1}\left(x_{0}\right)} \frac{d s}{a_{0}(s)}\right)\right|_{x_{0}=x_{0}^{*}}+O(\sqrt{\varepsilon}) .
\end{aligned}
$$

Итак, теперь у нас есть все необходимое для того, чтобы завершить доказательство леммы 4.2. Действительно, решения (4.52) выражаются через (4.58) 
посредством равенства

$$
\left[z^{(1)}(t, \varepsilon), z^{(2)}(t, \varepsilon)\right]=C^{-1}\left[\varkappa^{(1)}(t, \varepsilon), \varkappa^{(2)}(t, \varepsilon)\right]
$$

где (см. (4.59), (4.60))

$$
\begin{gathered}
C=\left.\left[\varkappa^{(1)}(t, \varepsilon), \varkappa^{(2)}(t, \varepsilon)\right]\right|_{t=-\delta}, \\
\operatorname{det} C=-\left.\frac{f(x(t, s), 0)}{f(s, 0)} g(x(t, s), 0)\right|_{t=-\delta, s=x_{0}^{*}}+O(\sqrt{\varepsilon}) \neq 0 .
\end{gathered}
$$

Подчеркнем, что определитель в (4.105) отличен от нуля в силу условия (3.139) на параметр $\delta$, неравенств $(2.4),(3.3)$ и того факта, что

$$
\left.x\left(t+T_{*}, s\right)\right|_{s=x_{1}^{*}}=\left.x(t, s)\right|_{s=x_{0}^{*}} \quad \forall t \in[-\delta, \delta] .
$$

Из установленных выше свойств решений (4.58) (см. (4.63), (4.80), (4.85), (4.86), (4.100), (4.103)) вытекают оценки

$$
\begin{gathered}
\max _{-h-\delta \leqslant t \leqslant-\delta}\left\|\varkappa^{(1)}\left(t+T_{*}(\varepsilon), \varepsilon\right)\right\| \leqslant \frac{M_{1}}{\varepsilon}, \\
\max _{-h-\delta \leqslant t \leqslant-\delta}\left\|\varkappa^{(2)}\left(t+T_{*}(\varepsilon), \varepsilon\right)\right\| \leqslant M_{2} \varepsilon^{-1 / 4}, \quad M_{1}, M_{2}=\text { const }>0 .
\end{gathered}
$$

Объединяя их с соотношениями (4.104), (4.105), для оператора (4.51) получаем требуемое неравенство (4.56).

Обратимся далее к матрице (4.55). Привлекая формулы (4.85), (4.103), (4.104) при $t=T_{*}(\varepsilon)-\delta$, нетрудно увидеть, что

$$
C^{-1} \mathscr{D}(\varepsilon) C=\left(\begin{array}{ll}
1 & r_{1} \\
0 & r_{2}
\end{array}\right)+O(\sqrt{\varepsilon}),
$$

где

$$
\begin{gathered}
r_{1}=\frac{x_{1}^{\prime}\left(x_{0}^{*}\right)}{f\left(x_{1}^{*}, 0\right)}-\frac{r_{2}}{f\left(x_{0}^{*}, 0\right)}, \\
r_{2}=\frac{f\left(x_{0}^{*}, 0\right)}{g\left(x_{0}^{*}, 0\right)}\left[\frac{g\left(x_{1}^{*}, 0\right)}{f\left(x_{1}^{*}, 0\right)} x_{1}^{\prime}\left(x_{0}^{*}\right)+\left.\frac{d}{d x_{0}}\left(\int_{x_{0}}^{x_{1}\left(x_{0}\right)} \frac{d s}{a_{0}(s)}\right)\right|_{x_{0}=x_{0}^{*}}\right]=\psi^{\prime}\left(x_{0}^{*}\right) .
\end{gathered}
$$

А отсюда очевидным образом следует, что собственные значения $\lambda_{1}(\varepsilon), \lambda_{2}(\varepsilon)$ матрицы $\mathscr{D}(\varepsilon)$ при $\varepsilon \rightarrow 0$ допускают асимптотику (4.57). Лемма 4.2 доказана.

Возвращаясь к исходному оператору $U(\varepsilon)$, заметим, что в силу равенств

$$
U(\varepsilon)=V(\varepsilon)+W(\varepsilon), \quad(\lambda I-U(\varepsilon))^{-1}=\left(I-(\lambda I-V(\varepsilon))^{-1} W(\varepsilon)\right)^{-1}(\lambda I-V(\varepsilon))^{-1}
$$

любое значение $\lambda \in \mathbb{C}$, для которого

$$
\left\|(\lambda I-V(\varepsilon))^{-1} W(\varepsilon)\right\|_{E \rightarrow E}<1,
$$


принадлежит резольвентному множеству этого оператора. Напомним далее, что оператор $W(\varepsilon)$ допускает оценку (4.54). В случае же $(\lambda I-V(\varepsilon))^{-1}$, опираясь на соотношения

$$
\begin{gathered}
(\lambda I-V(\varepsilon))^{-1} z_{0}(t)=\frac{1}{\lambda} z_{0}(t)+c_{1} \operatorname{colon}\left(z_{1}^{(1)}\left(T_{*}(\varepsilon)-\delta, \varepsilon\right), z_{2}^{(1)}\left(t+T_{*}(\varepsilon), \varepsilon\right)\right) \\
+c_{2} \operatorname{colon}\left(z_{1}^{(2)}\left(T_{*}(\varepsilon)-\delta, \varepsilon\right), z_{2}^{(2)}\left(t+T_{*}(\varepsilon), \varepsilon\right)\right), \quad-h-\delta \leqslant t \leqslant-\delta, \\
\left(\begin{array}{c}
c_{1} \\
c_{2}
\end{array}\right)=\frac{1}{\lambda}(\lambda I-\mathscr{D}(\varepsilon))^{-1} z_{0}(-\delta)
\end{gathered}
$$

и оценки (4.106), получаем неравенство

$$
\left\|(\lambda I-V(\varepsilon))^{-1}\right\|_{E \rightarrow E} \leqslant \frac{M\left(1+|\lambda|^{2}\right)}{\varepsilon|\lambda| \cdot\left|\lambda-\lambda_{1}\right| \cdot\left|\lambda-\lambda_{2}\right|} \quad \forall \lambda \in \mathbb{C}, \quad \lambda \neq 0, \lambda_{1}, \lambda_{2},
$$

где $M=$ const $>0, \lambda_{1}, \lambda_{2}$ - собственные значения матрицы (4.55).

На завершающем этапе исследования устойчивости цикла (4.13) объединим оценки (4.107), (4.108), (4.54) с асимптотическими представлениями (4.57). В результате убеждаемся, что любая точка $\lambda \in \mathbb{C}$ из множества

$$
\mathbb{C} \backslash\left\{O_{1} \cup O_{2} \cup O_{3}\right\},
$$

где

$$
\begin{gathered}
O_{1}=\left\{\lambda \in \mathbb{C}:|\lambda|<\exp \left(-\frac{M_{1}}{\varepsilon}\right)\right\}, \quad O_{2}=\left\{\lambda \in \mathbb{C}:\left|\lambda-\lambda_{1}\right|<\exp \left(-\frac{M_{2}}{\varepsilon}\right)\right\}, \\
O_{3}=\left\{\lambda \in \mathbb{C}:\left|\lambda-\lambda_{2}\right|<\exp \left(-\frac{M_{3}}{\varepsilon}\right)\right\},
\end{gathered}
$$

а постоянные $M_{j}>0, j=1,2,3$, подходящим образом малы, удовлетворяет условию (4.107) и, следовательно, является регулярной для оператора $U(\varepsilon)$. Что же касается спектра этого оператора, то он заведомо принадлежит шарам (4.109). А отсюда и из (4.57) требуемые соотношения (4.25), (4.26) вытекают очевидным образом, причем с необходимостью $\nu_{1}(\varepsilon) \equiv 1$ (поскольку система (4.20) - линеаризация на цикле).

Подведем итог. Второе асимптотическое представление (4.25) и соотношения (2.15), (4.26) приводят к выводу, что $\left|\nu_{k}(\varepsilon)\right|<1$ для всех $k \geqslant 2$. Тем самым, установлен факт экспоненциальной орбитальной устойчивости цикла (4.13) в метрике фазового пространства $\mathbb{R} \times C[-h-\delta,-\delta]$. Теорема 2.1 полностью доказана.

\section{§5. Некоторые приложения к экологии}

5.1. Математическая модель управляемого биоценоза. В настоящем пункте проиллюстрируем наши теоретические результаты на двух примерах из экологии. Первый из них представляет собой математическую модель биоценоза, находящегося под действием косвенного управления с обратной связью. 
В случае уравнения Хатчинсона (2.6) идея косвенного управления с обратной связью (indirect feedback control) реализована в статье [7] и монографии [8]. Суть этой идеи заключается в рассмотрении вместо уравнения (2.6) двухкомпонентной системы

$$
\dot{x}=-x+a y, \quad \varepsilon \dot{y}=(1-x-y(t-h)) y .
$$

Здесь $\varepsilon, a, h$ - положительные параметры, $x=x(t)>0$ - управляющий сигнал, который воздействует на популяцию с плотностью численности $y=y(t)$ и сам зависит от $y(t)$. Характер этой зависимости позволяет интерпретировать систему (5.1) как некий вариант задачи хищник-жертва.

В работах [7], [8] были найдены достаточные условия на параметры $\varepsilon, a, h$, при которых глобально устойчиво положение равновесия $x=a /(a+1), y=$ $1 /(a+1)$ системы (5.1). Мы же будем интересоваться ее автоколебательными процессами и покажем, что при $0<\varepsilon \ll 1$ и при соответствующем выборе запаздывания $h$ данная система удовлетворяет условиям 2.1-2.7.

В случае (5.1) имеем

$$
\begin{gathered}
f(x, y)=-x+a y, \quad g(x, y)=1-x, \\
a_{0}(x)=a, \quad b_{0}(x)=1-x, \quad q_{1}=q_{2}=1,
\end{gathered}
$$

а значит, условия 2.1-2.3 здесь выполняются автоматически. Далее обратим внимание, что в силу равенств $(5.2) \forall x_{0} \in(0,1)$ аналогичное $(2.8)$ уравнение $x-x^{2} / 2=x_{0}-x_{0}^{2} / 2$ допускает решение

$$
x_{1}=x_{1}\left(x_{0}\right)=2-x_{0}>1,
$$

а аналогичное (2.11) уравнение

$$
x-\ln x=2-x_{0}-\ln \left(2-x_{0}\right)+\frac{2}{a}\left(1-x_{0}\right)
$$

имеет на интервале $0<x<1$ единственное решение $x_{2}=x_{2}\left(x_{0}\right)$. Таким образом, корректно определено аналогичное (2.14) отображение

$$
x_{0} \in(0,1) \rightarrow \psi\left(x_{0}\right) \stackrel{\text { def }}{=} x_{2}\left(x_{0}\right) \in(0,1) .
$$

Покажем теперь, что отображение (5.5) допускает на множестве $0<x_{0}<1$ единственную неподвижную точку $x_{0}^{*}$. Для ее отыскания подставим в равенство (5.4) $x=x_{0}$. В результате убеждаемся, что $x_{0}^{*}$ является корнем уравнения

$$
P\left(x_{0}\right) \stackrel{\text { def }}{=} \ln \left(2-x_{0}\right)-\ln x_{0}-2\left(1+\frac{1}{a}\right)\left(1-x_{0}\right)=0 .
$$

Остановимся на некоторых свойствах функции $P\left(x_{0}\right)$. Нетрудно увидеть, что

$$
\begin{gathered}
P(1)=0, \quad P^{\prime}(1)=\frac{2}{a}>0, \quad P\left(x_{0}\right) \rightarrow+\infty \quad \text { при } \quad x_{0} \rightarrow+0, \\
x_{0}\left(2-x_{0}\right) P^{\prime}\left(x_{0}\right)=2\left(1+\frac{1}{a}\right) x_{0}\left(2-x_{0}\right)-2 .
\end{gathered}
$$


Отметим еще, что поскольку фигурирующий в правой части (5.8) квадратичный многочлен принимает разные знаки на концах отрезка $[0,1]$, то уравнение $P^{\prime}\left(x_{0}\right)=0$ имеет на интервале $0<x_{0}<1$ единственный корень $x_{0}=\widetilde{x}_{0}$, причем

$$
P^{\prime}\left(x_{0}\right)<0 \quad \text { при } 0<x_{0}<\widetilde{x}_{0}, \quad P^{\prime}\left(x_{0}\right)>0 \quad \text { при } \widetilde{x}_{0}<x_{0} \leqslant 1 .
$$

Установленные свойства (5.7), (5.9) свидетельствуют о том, что уравнение (5.6) на интересующем нас множестве $0<x_{0}<1$ допускает единственный корень $x_{0}=x_{0}^{*}$ такой, что

$$
P^{\prime}\left(x_{0}^{*}\right)<0 .
$$

Остается добавить, что этот корень является одновременно экспоненциально устойчивой неподвижной точкой отображения (5.5) (как показывает несложная проверка, неравенство $\psi^{\prime}\left(x_{0}^{*}\right)<1$ эквивалентно (5.10)).

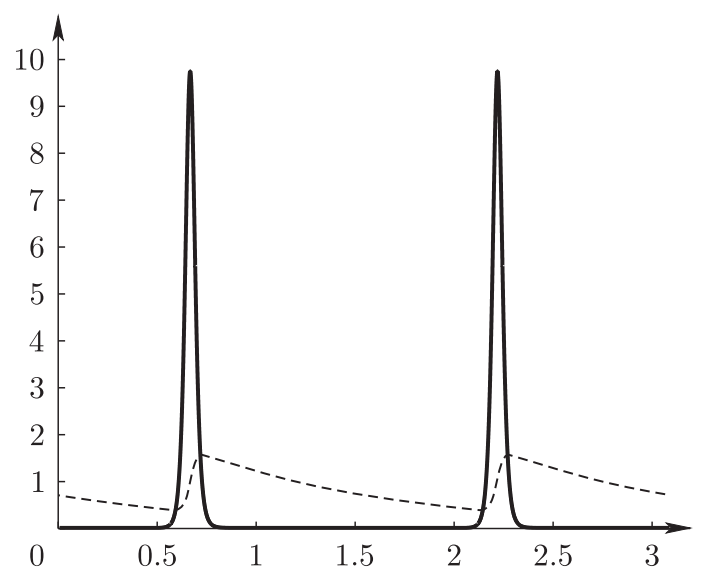

Рис. 6

Из проделанного анализа следует, что для системы (5.1) справедливы условия 2.4, 2.5. Сформулируем теперь ограничение на запаздывание $h$. Согласно (2.17) будем считать, что

$$
h<h_{*}=\ln \frac{x_{1}^{*}}{x_{3}^{*}},
$$

где $x_{1}^{*}=x_{1}\left(x_{0}^{*}\right)$ (см. $\left.(5.3)\right), x_{3}^{*}=x_{3}\left(x_{0}^{*}\right)$, а $x_{3}=x_{3}\left(x_{0}\right), x_{0} \in(0,1)$ - единственный корень аналогичного (5.4) уравнения

$$
x-\ln x=2-x_{0}-\ln \left(2-x_{0}\right)
$$

из интервала $0<x<1$. Что же касается оставшегося условия 2.7 , то в силу $(5.2)$ и свойств $x_{0}^{*} \in(0,1), x_{1}^{*}>1$ оно выполняется очевидным образом.

Итак, мы убедились, что при ограничении (5.11) на запаздывание $h$ система (5.1) подпадает под действие теоремы 2.1, а значит, допускает экспоненциально орбитально устойчивый неклассический релаксационный цикл. При $\varepsilon=0.01$, $a=2.1, h=1$ зависимость от времени его компонент $x, y$ представлена на рис. 6 (сплошной линией изображен график $y$, а пунктирной - график $x$ ). 
5.2. Модель динамики плотоядных-травоядных. Одна из первых математических моделей динамики взаимодействия плотоядных-травоядных, учитывающая эффекты запаздывания, была предложена в монографии [9]. Точнее говоря, в [9] рассматривалась система

$$
\dot{x}=(-1+a y) x, \quad \varepsilon \dot{y}=(1-x-y(t-h)) y, \quad \varepsilon, a, h>0,
$$

представляющая собой гибрид классической системы Лотки-Вольтерра с уравнением Хатчинсона. Более радикальную переработку модели Лотки-Вольтерра несколько позднее осуществил Ю.С. Колесов в статье [10]. Основная его идея состояла в том, что оба вида - и хищник, и жертва - должны меняться по хатчинсоновскому закону, а значит, их эволюция во времени должна описываться системой с двумя запаздываниями

$$
\dot{x}=\left(a y-x\left(t-h_{1}\right)\right) x, \quad \varepsilon \dot{y}=\left(1-x-y\left(t-h_{2}\right)\right) y,
$$

где $\varepsilon, a, h_{1}, h_{2}-$ положительные параметры.

Необходимо отметить, что система (5.12) не подходит для иллюстрации нашего основного результата (теоремы 2.1), так как соответствующее ей отображение $\psi:(0,1) \rightarrow(0,1)$ удовлетворяет неравенству $\psi\left(x_{0}\right)<x_{0}$ и, следовательно, не имеет неподвижных точек на интервале $0<x_{0}<1$. В связи с этим обратимся к системе

$$
\dot{x}=(a y-x) x, \quad \varepsilon \dot{y}=(1-x-y(t-h)) y,
$$

где $a, h=$ const $>0,0<\varepsilon \ll 1$, являющейся частным случаем (5.13).

Как и в предыдущем примере, условия 2.1-2.3 для системы (5.14) выполняются автоматически. Что же касается функций $x_{1}\left(x_{0}\right), x_{2}\left(x_{0}\right)$, то теперь первая из них - корень уравнения

$$
x-\ln x=x_{0}-\ln x_{0}, \quad x_{0} \in(0,1),
$$

лежащий на полуоси $x>1$, а вторая является корнем уравнения

$$
\frac{1}{x}+\ln x=\left.\left(\frac{1}{x_{1}}+\ln x_{1}+\frac{1}{a} \ln \frac{x_{1}}{x_{0}}\right)\right|_{x_{1}=x_{1}\left(x_{0}\right)}, \quad x_{0} \in(0,1),
$$

из интервала $0<x<1$. Таким образом, снова имеем дело с аналогичным $(5.5)$ отображением, переводящим интервал $0<x_{0}<1$ в себя.

Для нахождения неподвижной точки упомянутого отображения положим в (5.16) $x=x_{0}$ и учтем вытекающее из (5.15) равенство

$$
x_{1}-x_{0}=\ln \frac{x_{1}}{x_{0}} .
$$

В результате убеждаемся, что на неподвижной точке отображения (5.5) должно выполняться соотношение

$$
x_{1}=\frac{1}{x_{0}} \cdot \frac{a}{a+1} .
$$


Подставляя, далее, (5.18) в (5.17), для нахождения возможных значений $x_{0}$ получаем уравнение

$$
P\left(x_{0}\right) \stackrel{\text { def }}{=} \frac{1}{x_{0}} \cdot \frac{a}{a+1}-x_{0}+2 \ln x_{0}-\ln \frac{a}{a+1}=0 .
$$

Добавим еще, что в силу требования (5.18) и неравенства $x_{1}\left(x_{0}\right)>1$ уравнение (5.19) следует рассматривать только на интервале $0<x_{0}<a /(a+1)$.

Как и в предыдущем случае, выявим некоторые свойства функции $P\left(x_{0}\right)$ из (5.19). Непосредственная проверка показывает, что

$$
\begin{gathered}
P\left(\frac{a}{a+1}\right)=\ln \left(1-\frac{1}{a+1}\right)+\frac{1}{a+1}<0, \quad P^{\prime}\left(\frac{a}{a+1}\right)=\frac{1}{a}>0 \\
P\left(x_{0}\right) \rightarrow+\infty \text { при } x_{0} \rightarrow+0 \\
x_{0}^{2} P^{\prime}\left(x_{0}\right)=-x_{0}^{2}+2 x_{0}-\frac{a}{a+1} .
\end{gathered}
$$

Несложный анализ формул (5.20), (5.21) (см. аналогичное место выше) приводит к выводу о существовании у уравнения (5.19) на интервале $(0, a /(a+1))$ единственного корня $x_{0}=x_{0}^{*}$, удовлетворяющего неравенству $P^{\prime}\left(x_{0}^{*}\right)<0$, которое, в свою очередь, эквивалентно условию устойчивости $\psi^{\prime}\left(x_{0}^{*}\right)<1$ неподвижной точки $x_{0}=x_{0}^{*}$ отображения (5.5). Таким образом, убеждаемся, что для системы (5.14) выполняются условия 2.4, 2.5.

Для формулировки ограничения на запаздывание $h$ обратимся к уравнению

$$
\frac{1}{x}+\ln x=\left.\left(\frac{1}{x_{1}}+\ln x_{1}\right)\right|_{x_{1}=x_{1}\left(x_{0}\right)}, \quad x_{0} \in(0,1),
$$

и обозначим через $x=x_{3}\left(x_{0}\right)$ его корень из интервала $0<x<1$. Тогда требование (2.17) на запаздывание $h$ запишется в виде

$$
h<h_{*}=\frac{1}{x_{3}^{*}}-\frac{1}{x_{1}^{*}},
$$

где $x_{1}^{*}=x_{1}\left(x_{0}^{*}\right), x_{3}^{*}=x_{3}\left(x_{0}^{*}\right)$. Последнее же условие 2.7 здесь, как и для предыдущего примера, справедливо автоматически.

Из проделанных построений следует, что при ограничении (5.22) на запаздывание $h$ и при всех $0<\varepsilon \ll 1$ мы находимся в рамках применимости теоремы 2.1, гарантирующей существование у системы (5.14) экспоненциально орбитально устойчивого неклассического релаксационного цикла. Графики его компонент $x, y$ не приводим, так как они аналогичны показанным на рис. 6 .

\section{§6. Заключение}

Подводя итог, отметим, что нами построена теория неклассических релаксационных колебаний для систем с запаздыванием вида (1.25), которые можно рассматривать как модели задачи хищник-жертва. Однако аналог теоремы 2.1 сохраняется (вместе с доказательством) и для другого класса систем 
(1.25), в определенном смысле двойственного к рассмотренному выше. Системы из этого класса в биологической интерпретации соответствуют задаче жертва-хищник и удовлетворяют следующей серии ограничений.

УСловие 6.1. Считаем, что имеют место равенства (2.1), сохраняющие силу при дифференцировании по $x, y$ в любом порядке и любое число раз.

УСловиЕ 6.2. Предполагаем, что

$$
\begin{gathered}
a_{0}(x)<0 \quad \forall x>0 ; \\
b_{0}(x)<0 \quad \text { при } x \in\left(0, q_{1}\right), \quad b_{0}(x)>0 \quad \text { при } \quad x>q_{1},
\end{gathered}
$$

где $q_{1}=$ const $>0$.

Условие 6.3. Выполняются неравенства

$$
\begin{array}{lllll}
g(x, 0)<0 & \text { при } 0 \leqslant x<q_{2}, & g(x, 0)>0 & \text { при } x>q_{2} ; \\
f(x, 0)>0 & \text { при } 0<x<q_{3}, & f(x, 0)<0 & \text { при } x>q_{3},
\end{array}
$$

где постоянные $q_{2}, q_{3}>0$ таковы, что $q_{3}>\max \left(q_{1}, q_{2}\right)$.

Для формулировки очередного блока ограничений, как и ранее, рассмотрим функции (2.7).

УСловиЕ 6.4. Существует такое $x_{0}=x_{0}^{*} \in\left(\max \left(q_{1}, q_{2}\right), q_{3}\right)$, что уравнение (2.8) при $x_{0}=x_{0}^{*}$ имеет решение $x=x_{1}^{*} \in\left(0, \min \left(q_{1}, q_{2}\right)\right)$.

Опираясь на это условие и неравенства (6.1), (6.2), нетрудно увидеть, что к уравнению $(2.8)$ в точке $\left(x, x_{0}\right)=\left(x_{1}^{*}, x_{0}^{*}\right)$ применима теорема о неявной функции по переменной $x$. Таким образом, из (2.8) определяется функция $x=x_{1}\left(x_{0}\right), x_{1}\left(x_{0}^{*}\right)=x_{1}^{*}$, для которой имеет место аналогичное $(2.10)$ свойство

$$
x_{1}\left(x_{0}\right) \in\left(0, \min \left(q_{1}, q_{2}\right)\right) \quad \forall x_{0} \in[a, b],
$$

где $[a, b] \subset\left(\max \left(q_{1}, q_{2}\right), q_{3}\right)$ - отрезок достаточно малой длины, содержащий внутри себя точку $x_{0}^{*}$.

Далее рассмотрим уравнение (2.11), где переменная $x_{0}$ пробегает отрезок $[a, b]$ из $(6.5)$, а $x_{1}=x_{1}\left(x_{0}\right)$. При анализе этого уравнения полезно иметь в виду, что здесь вместо (2.12) в силу (6.3), (6.4) справедливы соотношения

$$
\begin{gathered}
\frac{\partial \gamma_{1}}{\partial x}\left(x, x_{1}\right)<0 \quad \text { при } x \in\left(0, q_{2}\right), \quad \frac{\partial \gamma_{1}}{\partial x}\left(x, x_{1}\right)>0 \quad \text { при } x>q_{2}, \\
\gamma_{1}\left(x, x_{1}\right) \rightarrow+\infty \quad \text { при } \quad x \rightarrow q_{3}-0 .
\end{gathered}
$$

Объединяя свойства (6.6) с оценкой $x_{1}<q_{2}$ и неравенством (2.13) (которое в данном случае сохраняется, так как $\left.a_{0}(x)<0, x_{1}<x_{0}\right)$, приходим к выводу, что уравнение (2.11) имеет на интервале $q_{2}<x<q_{3}$ единственное решение $x=x_{2}\left(x_{0}\right)$. А это значит, что на отрезке $a \leqslant x_{0} \leqslant b$ определено отображение вида (2.14), причем, как нетрудно проверить, $\psi^{\prime}\left(x_{0}\right)>0 \forall x_{0} \in[a, b]$. 
УСловие 6.5. Предполагаем, что точка $x_{0}=x_{0}^{*}$ из условия 6.4 удовлетворяет соотношениям (2.15).

Условие 6.6. Считаем, что

$$
h<h_{*} \stackrel{\text { def }}{=} \int_{x_{1}^{*}}^{x_{3}^{*}} \frac{d s}{f(s, 0)},
$$

где $x_{3}^{*}=x_{3}\left(x_{0}^{*}\right)$, а функция $x=x_{3}\left(x_{0}\right) \in\left(q_{2}, q_{3}\right), x_{3}\left(x_{0}\right)<x_{2}\left(x_{0}\right)-$ корень уравнения (2.16) при $x_{1}=x_{1}\left(x_{0}\right)$.

УСловие 6.7. В точках $x=x_{0}^{*}$ и $x=x_{1}^{*}$, о которых идет речь в условии 6.4 , имеют место неравенства (2.18).

Перечисленные ограничения позволяют применить к системе (1.25) разработанные выше методы исследования неклассических релаксационных колебаний и получить следующее утверждение.

ТеОРема 6.1. При выполнении условий 6.1-6.7 и при всех достаточно малъх $\varepsilon>0$ система (1.25) допускает экспоненциалъно орбиталъно устойчивыи иикл $\left(x_{*}(t, \varepsilon), y_{*}(t, \varepsilon)\right), y_{*}(t, \varepsilon)>0, y_{*}(0, \varepsilon) \equiv 1$ периода $T_{*}(\varepsilon)$, обладающий аналогичными (2.21)-(2.24) свойствами.

В качестве примера на применение теоремы 6.1 приведем аналогичную (1.1) систему

$\dot{x}=\alpha-x(y+1), \quad \varepsilon \dot{y}=(x-\beta-y(t-h)) y, \quad \alpha, \beta, h=$ const $>0, \quad 0<\varepsilon \ll 1$,

из теории лазеров [11]. Покажем, что при $\alpha>\beta$ и при подходящем уменьшении запаздывания $h$ она удовлетворяет условиям 6.1-6.7.

В случае системы (6.8) в силу равенств

$$
\begin{gathered}
f(x, y)=\alpha-x(y+1), \quad g(x, y)=b_{0}(x)=x-\beta, \quad a_{0}(x)=-x, \\
q_{1}=q_{2}=\beta, \quad q_{3}=\alpha
\end{gathered}
$$

условия 6.1-6.3 выполняются автоматически. В проверке нуждаются лишь требуемые в условиях $6.4,6.5$ свойства отображения $\psi\left(x_{0}\right)$, которое задается следующим образом.

Фиксируем произвольно $x_{0} \in(\beta, \alpha)$ и обозначим через $x_{1}=x_{1}\left(x_{0}\right)$ единственный корень уравнения

$$
x-\beta \ln x=x_{0}-\beta \ln x_{0}
$$

из интервала $0<x<\beta$. Далее, рассмотрим уравнение

$$
x-x_{1}+(\alpha-\beta) \ln \frac{\alpha-x}{\alpha-x_{1}}=\ln \frac{x_{1}}{x_{0}}
$$

при $x_{1}=x_{1}\left(x_{0}\right)$, а через $x_{2}=x_{2}\left(x_{0}\right)$ обозначим единственный его корень, лежащий на интервале $\beta<x<\alpha$. И, наконец, положим

$$
x_{0} \in(\beta, \alpha) \rightarrow \psi\left(x_{0}\right) \stackrel{\text { def }}{=} x_{2}\left(x_{0}\right) \in(\beta, \alpha) .
$$


Отыскание возможных неподвижных точек отображения (6.11) проводится по схеме, описанной при доказательстве леммы 1.2. Поэтому здесь лишь вкратце остановимся на ее основных моментах. Сначала, полагая в (6.9), (6.10) $x=x_{1}$ и $x=x_{0}$ соответственно, для определения $x_{0}, x_{1}$ приходим к аналогичной (1.15) системе

$$
x_{1}-x_{0}=\beta \ln \frac{x_{1}}{x_{0}}, \quad x_{0}-x_{1}=(\alpha-\beta) \ln \frac{\alpha-x_{1}}{\alpha-x_{0}}+\ln \frac{x_{1}}{x_{0}} .
$$

Далее, введем в рассмотрение величину z, задающуюся формулой (1.16), и, опираясь на соотношения (6.12), выразим компоненты $x_{0}, x_{1}$ через $z$. В итоге убеждаемся, что имеют место аналогичные (1.17) равенства

$$
x_{0}=\frac{\alpha(z-1) z^{(\alpha-\beta) /(\beta+1)}}{z^{(\alpha+1) /(\beta+1)}-1}, \quad x_{1}=\frac{\alpha(z-1)}{z^{(\alpha+1) /(\beta+1)}-1},
$$

а переменная $z$ является корнем аналогичного (1.18) уравнения

$$
P(z) \stackrel{\text { def }}{=} \frac{(\alpha-\beta) \beta}{\beta+1}\left(z^{(\alpha+1) /(\beta+1)}-1\right) \ln z+\alpha(z-1)\left(1-z^{(\alpha-\beta) /(\beta+1)}\right)=0 .
$$

Последующий анализ, связанный с уравнением (6.14), идентичен соответствующему фрагменту обоснования леммы 1.2. Поэтому приведем сразу окончательный результат: на полуоси $z>1$ данное уравнение имеет единственный корень $z=z_{*}$, удовлетворяющий неравенству (1.20), а найденные по $z=z_{*}$ с помощью соотношений (6.13) точки $x_{0}^{*}$ и $x_{1}^{*}$ удовлетворяют условиям $6.4,6.5,6.7$. В частности, требование $\psi^{\prime}\left(x_{0}^{*}\right)<1$ заведомо выполняется, так как совпадает c (1.20).

Суммируя проделанные построения и опираясь на теорему 6.1, приходим к выводу, что при $\alpha>\beta$ и при условии вида (6.7) на запаздывание $h$ у системы (6.8) существует экспоненциально орбитально устойчивый неклассический релаксационный цикл. Графики компонент $x, y$ этого цикла имеют вид, аналогичный показанному на рис. 4.

\section{Список литературы}

[1] Е. Ф. Мищенко, Н. Х. Розов, Дифференциалъные уравнения с малым параметром и релаксационные колебания, Наука, М., 1975, 247 с.; англ. пер.: E. F. Mishchenko, N. Kh. Rozov, Differential equations with small parameters and relaxation oscillations, Math. Concepts Methods Sci. Engrg., 13, Plenum Press, New York, 1980, x+228 pp.

[2] Е.Ф. Мищенко, Ю.С. Колесов, А. Ю. Колесов, Н.Х. Розов, Периодические движения и бифуркационные процессы в сингулярно возмущенных системах, Физматлит, М., 1995, 336 с.; англ. пер.: E. F. Mishchenko, Yu.S. Kolesov, A. Yu. Kolesov, N. Kh. Rozov, Asymptotic methods in singularly perturbed systems, Monogr. Contemp. Math., Consultants Bureau, New York, 1994, xii+281 pp.

[3] А.Ю. Колесов, "Специфические релаксационные циклы систем типа ЛоткиВольтерра", Изв. АН СССР. Сер. матем., 55:3 (1991), 515-536; англ. пер.: A. Yu. Kolesov, "Specific relaxation cycles of systems of Lotka-Volterra type", Math. USSR-Izv., 38:3 (1992), 503-523. 
[4] С. Д. Глызин, А. Ю. Колесов, Н. Х. Розов, "Релаксационные колебания и диффузионный хаос в реакции Белоусова", Ж. вычисл. матем. и матем. физ., 51:8 (2011), 1400-1418; англ. пер.: S. D. Glyzin, A. Yu. Kolesov, N. Kh. Rozov, "Relaxation oscillations and diffusion chaos in the Belousov reaction", Comput. Math. Math. Phys., 51:8 (2011), 1307-1324.

[5] Я.И. Ханин, Основы динамики лазеров, Физматлит, М., 1999, 368 с.

[6] G. E. Hutchinson, "Circular causal systems in ecology", Ann. New York Acad. Sci., 50 (1948), 221-246.

[7] K. Gopalsamy, Pei-Xuan Weng, "Feedback regulation of logistic growth", Internat. J. Math. Math. Sci., 16:1 (1993), 177-192.

[8] K. Gopalsamy, Stability and oscillations in delay differential equations of population dynamics, Math. Appl., 74, Kluwer Academic Publishers Group, Dordrecht, 1992, xii+501 pp.

[9] R. M. May, Stability and complexity in model ecosystems, Princeton Univ. Press, Princeton, NJ, 1973, 235 pp.

[10] Ю. С. Колесов, "Резонансы в экологии", Исследования по устойчивости и теории колебаний, ЯрГУ, Ярославль, 1978, $26-42$.

[11] С. А. Кащенко, Е. В. Григорьева, Релаксачионные колебания в лазерах, Синергетика: от прошлого к будущему, 64, URSS, M., 2013, 266 с.

Сергей Дмитриевич Глызин

(Sergey D. Glyzin)

Ярославский государственный университет

им. П. Г. Демидова

E-mail: glyzin@uniyar.ac.ru

\section{Андрей Юрьевич Колесов}

(Andrei Yu. Kolesov)

Ярославский государственный университет

им. П. Г. Демидова

E-mail: kolesov@uniyar.ac.ru

\section{Николай Христович Розов}

(Nikolai Kh. Rozov)

Московский государственный университет

им. М. В. Ломоносова

E-mail: fpo.mgu@mail.ru
Поступила в редакцию 17.07.2013 